\title{
MIXED TYPE MULTIPLE ORTHOGONAL POLYNOMIALS FOR TWO NIKISHIN SYSTEMS
}

\author{
U. FIDALGO PRIETO, A. LÓPEZ GARCÍA, G. LÓPEZ LAGOMASINO, AND V. N. SOROKIN
}

\begin{abstract}
We study the logarithmic and ratio asymptotics of linear forms constructed from a Nikishin system which satisfy orthogonality conditions with respect to a system of measures generated by a second Nikishin system. This construction combines type I and type II multiple orthogonal polynomials. The logarithmic asymptotics of the linear forms is expressed in terms of the extremal solution of an associated vector valued equilibrium problem for the logarithmic potential. The ratio asymptotics is described by means of a conformal representation of an appropriate Riemann surface of genus zero onto the extended complex plane.
\end{abstract}

Keywords and phrases. Nikishin system, multiple orthogonal polynomials, logarithmic asymptotics, rate of convergence, potential theory, ratio asymptotics.

A.M.S. Subject Classification. Primary: 30E10, 42C05; Secondary: 41A20.

\section{INTRODUCTION}

Let $s$ be a finite positive Borel measure supported on a compact subset $\operatorname{supp}(s)$ of the real line, and $\left(w_{0}^{1}, \ldots, w_{m_{1}}^{1}\right),\left(w_{0}^{2}, \ldots, w_{m_{2}}^{2}\right)$ be two systems of continuous functions on $\operatorname{supp}(s)$. Fix $\mathbf{n}_{1}=\left(n_{1,0}, n_{1,1}, \ldots, n_{1, m_{1}}\right) \in \mathbb{Z}_{+}^{m_{1}+1}$ and $\mathbf{n}_{2}=\left(n_{2,0}, n_{2,1}, \ldots, n_{2, m_{2}}\right) \in \mathbb{Z}_{+}^{m_{2}+1}$. Set $\left|\mathbf{n}_{1}\right|=$ $n_{1,0}+n_{1,1}+\cdots+n_{1, m_{1}},\left|\mathbf{n}_{2}\right|=n_{2,0}+\cdots+n_{2, m_{2}}$, and $\mathbf{n}=\left(\mathbf{n}_{1} ; \mathbf{n}_{2}\right)$. In the sequel, we suppose that $\left|\mathbf{n}_{2}\right|+1=\left|\mathbf{n}_{1}\right|$.

Let $\left|\mathbf{n}_{1}\right| \geq 1$. It is easy to see that there exist polynomials $a_{\mathbf{n}, 0}, a_{\mathbf{n}, 1}, \ldots, a_{\mathbf{n}, m_{1}}$ such that:

i) $\operatorname{deg}\left(a_{\mathbf{n}, j}\right) \leq n_{1, j}-1, j=0, \ldots, m_{1}$, not all identically equal to zero.

ii) For $k=0, \ldots, m_{2}$

$$
\int x^{\nu} \sum_{j=0}^{m_{1}} a_{\mathbf{n}, j}(x) w_{j}^{1}(x) w_{k}^{2}(x) d s(x)=0, \quad \nu=0, \ldots, n_{2, k}-1 .
$$

$\left(\operatorname{deg}\left(a_{\mathbf{n}, j}\right) \leq-1\right.$ means that $\left.a_{\mathbf{n}, j} \equiv 0.\right)$

When $m_{2}=0$ the polynomials $\left(a_{\mathbf{n}, 0}, \ldots, a_{\mathbf{n}, m_{1}}\right)$ are called type I multiple orthogonal polynomials. If $m_{1}=0, a_{\mathbf{n}, 0}$ is called a type II multiple orthogonal polynomial. The case $m_{1}=m_{2}=0$

The first three authors in alphabetical order were supported by grants MTM 2006-13000-C03-02 of Ministerio de Ciencia y Tecnología and CCG07-UC3M/ESP-3339 of Comunidad Autónoma de Madrid-Universidad Carlos III de Madrid. V. N. Sorokin received support from grants RFBR-08-01-00317 and NSh-3906.2008.1. 
reduces to the usual definition of orthogonal polynomial. When $m_{1}, m_{2} \geq 1$ these multiple orthogonal polynomials are called of mixed type.

Multiple orthogonal polynomials appear in problems connected with the algebraic independence of functions and numbers (type I) and in questions related with simultaneous rational approximation (type II). Those of type II are formed by polynomials which share orthogonality conditions with a system of measures which may be written in the form of orthogonality relations with respect to a family of generalized polynomials (that in the sequel we call linear forms). In type I, the linear forms are defined through full orthogonality relations with respect to a single measure. Mixed type multiple orthogonal polynomials occur in stochastic models connected with random matrices and non intersecting random paths, see [6]. Mixed type multiple orthogonal polynomials as presented above were considered in [24] and their algebraic properties studied in [26].

We will restrict our attention to mixed type multiple orthogonal polynomials in which the linear forms are generated by two (not necessarily distinct) Nikishin systems of measures. Nikishin systems of measures were introduced in [18]. Before going into details let us mention some papers which constitute our starting point.

E. M. Nikishin studied the asymptotic behavior of the linear forms generated by a Nikishin system of measures in [19] (see also [15] and the last section in [20]). He described the logarithmic asymptotics of type I multiple orthogonal polynomials in terms of the solution of a vector equilibrium problem for the logarithmic potential. Later, Gonchar-Rakhmanov-Sorokin studied in [11] the rate of convergence of Hermite-Padé approximation of generalized Nikishin systems of functions and the logarithmic asymptotics of their associated type II multiple orthogonal polynomials. The solution is also characterized by a similar vector equilibrium problem. In [25], V. N. Sorokin defines mixed type multiple orthogonal polynomials for two Nikishin systems and gives their logarithmic asymptotics.

Let $s$ be a finite positive Borel measure supported on a bounded interval $\Delta$ of the real line $\mathbb{R}$ such that $s^{\prime}>0$ almost everywhere on $\Delta$ and let $\left\{Q_{n}\right\}, n \in \mathbb{Z}_{+}$, be the corresponding sequence of monic orthogonal polynomials; that is, with leading coefficients equal to one. In a series of two papers (see [21] and [22]), E. A. Rakhmanov proved that under these assumptions

$$
\lim _{n \in \mathbb{Z}_{+}} \frac{Q_{n+1}(z)}{Q_{n}(z)}=\frac{\varphi(z)}{\varphi^{\prime}(\infty)}, \quad \mathcal{K} \subset \mathbb{C} \backslash \Delta
$$

(uniformly on each compact subset of $\mathbb{C} \backslash \Delta$ ), where $\varphi(z)$ denotes the conformal representation of $\overline{\mathbb{C}} \backslash \Delta$ onto $\{w:|w|>1\}$ such that $\varphi(\infty)=\infty$ and $\varphi^{\prime}(\infty)>0$. This result attracted great attention because of its theoretical interest within the general theory of orthogonal polynomials and its applications to the theory of rational approximation of analytic functions. Simplified proofs of Rakhmanov's theorem may be found in [23] and [16].

This result has been extended in several directions. Orthogonal polynomials with respect to varying measures (depending on the degree of the polynomial) arise in the study of multipoint Padé 
approximation of Markov functions. In this context, in [12] and [13], an analogue of Rakhmanov's theorem for such sequences of orthogonal polynomials was proved. Recently, S. A. Denisov [7] (see also [17]) extended Rakhmanov's result to the case when $\operatorname{supp}(s)=\widetilde{\Delta} \cup e \subset \mathbb{R}$, where $\widetilde{\Delta}$ is a bounded interval, $e$ is a set without accumulation points in $\widetilde{\mathbb{R}} \backslash \widetilde{\Delta}$, and $s^{\prime}>0$ a.e. on $\widetilde{\Delta}$. A version for orthogonal polynomials with respect to varying Denisov type measures was given in [2].

For multiple orthogonal polynomials associated with Nikishin systems of measures an analogue of Rakhmanov's theorem was proved in [1] and extended in [14] to the case when the measures in the Nikishin system are as those considered by Denisov.

Let us define the notion of Nikishin system of measures. Let $\sigma_{\alpha}, \sigma_{\beta}$ be two measures with constant sign supported on $\mathbb{R}$ and let $\Delta_{\alpha}, \Delta_{\beta}$ denote the smallest intervals containing their supports, $\operatorname{supp}\left(\sigma_{\alpha}\right)$ and $\operatorname{supp}\left(\sigma_{\beta}\right)$, respectively. We write $\operatorname{Co}\left(\operatorname{supp}\left(\sigma_{\alpha}\right)\right)=\Delta_{\alpha}$. Assume that $\Delta_{\alpha} \cap \Delta_{\beta}=\emptyset$ and define

$$
\left\langle\sigma_{\alpha}, \sigma_{\beta}\right\rangle(x):=\int \frac{d \sigma_{\beta}(t)}{x-t} d \sigma_{\alpha}(x)=\widehat{\sigma}_{\beta}(x) d \sigma_{\alpha}(x) .
$$

Therefore, $\left\langle\sigma_{\alpha}, \sigma_{\beta}\right\rangle$ is a measure with constant sign and support equal to that of $\sigma_{\alpha}$.

For a system of intervals $\Delta_{0}, \ldots, \Delta_{m}$ contained in $\mathbb{R}$ satisfying $\Delta_{j} \cap \Delta_{j+1}=\emptyset, j=0, \ldots, m-1$, and finite Borel measures $\sigma_{0}, \ldots, \sigma_{m}$ with constant sign in $\operatorname{Co}\left(\operatorname{supp}\left(\sigma_{j}\right)\right)=\Delta_{j}$, such that each one has infinitely many points in its support, we define recursively

$$
\left\langle\sigma_{0}, \sigma_{1}, \ldots, \sigma_{j}\right\rangle=\left\langle\sigma_{0},\left\langle\sigma_{1}, \ldots, \sigma_{j}\right\rangle\right\rangle, \quad j=1, \ldots, m .
$$

We say that $\left(s_{0}, \ldots, s_{m}\right)=\mathcal{N}\left(\sigma_{0}, \ldots, \sigma_{m}\right)$, where

$$
s_{0}=\left\langle\sigma_{0}\right\rangle=\sigma_{0}, \quad s_{1}=\left\langle\sigma_{0}, \sigma_{1}\right\rangle, \ldots, \quad s_{m}=\left\langle\sigma_{0}, \ldots, \sigma_{m}\right\rangle
$$

is the Nikishin system of measures generated by $\left(\sigma_{0}, \ldots, \sigma_{m}\right)$. In the sequel, when referring to a Nikishin system the condition $\Delta_{j} \cap \Delta_{j+1}=\emptyset, j=0, \ldots, m-1$, is always assumed to hold. Notice that all the measures in a Nikishin system have the same support, namely $\operatorname{supp}\left(\sigma_{0}\right)$. We will denote $\left(s_{j, j}=\sigma_{j}\right)$

$$
s_{j, k}=\left\langle\sigma_{j}, \ldots, \sigma_{k}\right\rangle, \quad 0 \leq j \leq k \leq m .
$$

Take two systems $S^{1}=\left(s_{0}^{1}, \ldots, s_{m_{1}}^{1}\right)=\mathcal{N}\left(\sigma_{0}^{1}, \ldots, \sigma_{m_{1}}^{1}\right), S^{2}=\left(s_{0}^{2}, \ldots, s_{m_{2}}^{2}\right)=\mathcal{N}\left(\sigma_{0}^{2}, \ldots, \sigma_{m_{2}}^{2}\right)$ generated by $m_{1}+1$ and $m_{2}+1$ measures, respectively. The two systems need not coincide, but we will assume that $\sigma_{0}^{1}=\sigma_{0}^{2}$; that is, both systems stem from the same basis measure. The smallest interval containing $\operatorname{supp}\left(\sigma_{j}^{i}\right)$ will be denoted $\operatorname{Co}\left(\operatorname{supp}\left(\sigma_{j}^{i}\right)\right)=\Delta_{j}^{i}$.

Fix $\mathbf{n}_{1}=\left(n_{1,0}, n_{1,1}, \ldots, n_{1, m_{1}}\right) \in \mathbb{Z}_{+}^{m_{1}+1}$ and $\mathbf{n}_{2}=\left(n_{2,0}, n_{2,1}, \ldots, n_{2, m_{2}}\right) \in \mathbb{Z}_{+}^{m_{2}+1}$. Set $\left|\mathbf{n}_{1}\right|=$ $n_{1,0}+n_{1,1}+\cdots+n_{1, m_{1}},\left|\mathbf{n}_{2}\right|=n_{2,0}+\cdots+n_{2, m_{2}}$, and $\mathbf{n}=\left(\mathbf{n}_{1} ; \mathbf{n}_{2}\right)$. We will always assume that

$$
\left|\mathbf{n}_{2}\right|+1=\left|\mathbf{n}_{1}\right|
$$

Definition 1.1. Let $\left|\mathbf{n}_{1}\right| \geq 1$. The system of polynomials $a_{\mathbf{n}, 0}, a_{\mathbf{n}, 1}, \ldots, a_{\mathbf{n}, m_{1}}$ satisfying:

i') $\operatorname{deg}\left(a_{\mathbf{n}, j}\right) \leq n_{1, j}-1, j=0, \ldots, m_{1}$, not all identically equal to zero. 
ii') For $k=0, \ldots, m_{2}$

$$
\int x^{\nu}\left(a_{\mathbf{n}, 0}(x)+\sum_{j=1}^{m_{1}} a_{\mathbf{n}, j}(x) \widehat{s}_{1, j}^{1}(x)\right) d s_{0, k}^{2}(x)=0, \quad \nu=0, \ldots, n_{2, k}-1,
$$

$\left(\operatorname{deg}\left(a_{\mathbf{n}, j}\right) \leq-1\right.$ means that $\left.a_{\mathbf{n}, j} \equiv 0\right)$ is called a system of mixed type multiple orthogonal polynomials relative to the multi-index $\mathbf{n}=\left(\mathbf{n}_{1} ; \mathbf{n}_{2}\right)$ and the pair $\left(S^{1}, S^{2}\right)$ of Nikishin systems. The vector of polynomials $\left(a_{\mathbf{n}, 0}, \ldots, a_{\mathbf{n}, m_{1}}\right)$ is said to be "monic" if its last entry different from zero has leading coefficient equal to 1 . A multi-index $\mathbf{n}=\left(\mathbf{n}_{1} ; \mathbf{n}_{2}\right)$ is said to be normal if every solution to $\left.\left.i^{\prime}\right)-i i^{\prime}\right)$ satisfies $\operatorname{deg} a_{\mathbf{n}, j}=n_{1, j}-1, j=0, \ldots, m_{1}$.

The concept of mixed multiple orthogonal polynomial with respect to a pair of Nikishin systems was first introduced by V. N. Sorokin in [25].

Finding $a_{\mathbf{n}, 0}, \ldots, a_{\mathbf{n}, m_{1}}$ reduces to solving a homogeneous linear system of $\left|\mathbf{n}_{2}\right|$ equations on $\left|\mathbf{n}_{1}\right|$ unknowns. Since $\left|\mathbf{n}_{2}\right|=\left|\mathbf{n}_{1}\right|-1$ a non-trivial solution is guaranteed. If $\mathbf{n}$ is normal, it is easy to verify that the vector $\left(a_{\mathbf{n}, 0}, \ldots, a_{\mathbf{n}, m_{1}}\right)$ is uniquely determined except for a constant factor, and in that case the "monic" vector is unique. Set

$$
\mathbb{Z}_{+}^{m_{1}+1}(\bullet)=\left\{\mathbf{n}_{1} \in \mathbb{Z}_{+}^{m_{1}+1}: n_{1,0} \geq \cdots \geq n_{1, m_{1}}\right\}
$$

In Proposition 2.3, we prove that all $\mathbf{n}=\left(\mathbf{n}_{1} ; \mathbf{n}_{2}\right) \in \mathbb{Z}_{+}^{m_{1}+1}(\bullet) \times \mathbb{Z}_{+}^{m_{2}+1}(\bullet)$ are normal. For the sequences of multi-indices we shall consider, for almost all $\mathbf{n}$ we will have that $n_{1, m_{1}} \geq 1$ and a "monic" $\left(a_{\mathbf{n}, 0}, a_{\mathbf{n}, 1}, \ldots, a_{\mathbf{n}, m_{1}}\right)$ will have $a_{\mathbf{n}, m_{1}}$ monic.

Theorem 1 gives the rate of convergence of the $\left|\mathbf{n}_{1}\right|$-th root of the linear forms

$$
\mathcal{A}_{\mathbf{n}, 0}(z)=a_{\mathbf{n}, 0}(z)+\sum_{k=1}^{m_{1}} a_{\mathbf{n}, k}(z) \widehat{s}_{1, k}^{1}(z),
$$

under mild conditions on the sequence of multi-indices and the measures generating both Nikishin systems. A measure $\sigma$ is said to be regular if

$$
\lim _{n \rightarrow \infty} \kappa_{n}^{1 / n}=1 / \operatorname{cap}(\operatorname{supp}(\sigma)),
$$

where $\operatorname{cap}(\cdot)$ denotes the logarithmic capacity of the Borel set $(\cdot)$ and $\kappa_{n}$ denotes the leading coefficient of the $n$th orthonormal polynomial with respect to $\sigma$. For different equivalent forms of defining regular measures see sections 3.1 to 3.3 in [28] (in particular Theorem 3.1.1). For short, we write $\left(S^{1}, S^{2}\right) \in \mathbf{R e g}$ to mean that all the measures which generate both Nikishin systems are regular and their supports are regular compact sets. Recall that a compact set is regular when the Green's function with singularity at $\infty$ of the unbounded connected component of the complement of the compact set can be extended continuously to all $\mathbb{C}$. Before stating Theorem 1, we need to introduce some notation and results from potential theory.

Let $E_{k}, k=-m_{2}, \ldots, m_{1}$, be (not necessarily distinct) compact subsets of the real line and $\mathcal{C}=\left(c_{j, k}\right),-m_{2} \leq j, k \leq m_{1}$, a real, positive definite, symmetric matrix of order $m_{1}+m_{2}+1 . \mathcal{C}$ 
will be called the interaction matrix. By $\mathcal{M}\left(E_{k}\right)$ we denote the class of all finite, positive, Borel measures with compact support consisting of an infinite set of points contained in $E_{k}$ and $\mathcal{M}_{1}\left(E_{k}\right)$ is the subclass of probability measures of $\mathcal{M}\left(E_{k}\right)$. Set

$$
\mathcal{M}_{1}=\mathcal{M}_{1}\left(E_{-m_{2}}\right) \times \cdots \times \mathcal{M}_{1}\left(E_{m_{1}}\right) .
$$

Given a vector measure $\mu=\left(\mu_{-m_{2}}, \ldots, \mu_{m_{1}}\right) \in \mathcal{M}_{1}$ and $j=-m_{2}, \ldots, m_{1}$, we define the combined potential

$$
W_{j}^{\mu}(x)=\sum_{k=-m_{2}}^{m_{1}} c_{j, k} V^{\mu_{k}}(x)
$$

where

$$
V^{\mu_{k}}(x)=\int \log \frac{1}{|x-t|} d \mu_{k}(t)
$$

denotes the standard logarithmic potential of $\mu_{k}$. We denote

$$
\omega_{j}^{\mu}=\inf \left\{W_{j}^{\mu}(x): x \in E_{j}\right\}, \quad j=-m_{2}, \ldots, m_{1}
$$

In Chapter 5 of [20] the authors prove (we state the result in a form convenient for our purpose).

Lemma 1.2. Assume that the compact sets $E_{k}, k=-m_{2}, \ldots, m_{1}$, are regular with respect to the Dirichlet problem. Let $\mathcal{C}$ be a real, positive definite, symmetric matrix of order $m_{1}+m_{2}+1$. If there exists $\bar{\mu}=\left(\bar{\mu}_{-m_{2}}, \ldots, \bar{\mu}_{m_{1}}\right) \in \mathcal{M}_{1}$ such that for each $j=-m_{2}, \ldots, m_{1}$

$$
W_{j}^{\bar{\mu}}(x)=\omega_{j}^{\bar{\mu}}, \quad x \in \operatorname{supp}\left(\bar{\mu}_{j}\right),
$$

then $\bar{\mu}$ is unique. Moreover, if $c_{j, k} \geq 0$ when $E_{j} \cap E_{k} \neq \emptyset$, then $\bar{\mu}$ exists.

For details on how this lemma is derived from [20, Chapter 5] see [3, Section 4]. The vector measure $\bar{\mu} \in \mathcal{M}_{1}$ is called the equilibrium solution for the vector potential problem determined by the interaction matrix $\mathcal{C}$ on the system of compact sets $E_{j}, j=-m_{2}, \ldots, m_{1}$.

Let $\Lambda=\Lambda\left(p_{1,0}, \ldots, p_{1, m_{1}} ; p_{2,0}, \ldots, p_{2, m_{2}}\right) \subset \mathbb{Z}_{+}^{m_{1}+1}(\bullet) \times \mathbb{Z}_{+}^{m_{2}+1}(\bullet)$ be an infinite sequence of distinct multi-indices such that

$$
\lim _{\mathbf{n} \in \Lambda} \frac{n_{1, j}}{\left|\mathbf{n}_{1}\right|}=p_{1, j} \in(0,1), \quad j=0, \ldots, m_{1}, \quad \lim _{\mathbf{n} \in \Lambda} \frac{n_{2, j}}{\left|\mathbf{n}_{2}\right|}=p_{2, j} \in(0,1), \quad j=0, \ldots, m_{2} .
$$

Obviously, $p_{1,0} \geq \cdots \geq p_{1, m_{1}}, p_{2,0} \geq \cdots \geq p_{2, m_{2}}$, and $\sum_{j=0}^{m_{1}} p_{1, j}=\sum_{j=0}^{m_{2}} p_{2, j}=1$. Set

$$
P_{j}=\sum_{k=j}^{m_{1}} p_{1, k}, j=0, \ldots, m_{1}, \quad P_{-j}=\sum_{k=j}^{m_{2}} p_{2, k}, j=0, \ldots, m_{2} .
$$


Let us define the interaction matrix $\mathcal{C}$ which is relevant for the rest of the paper. Take the tri-diagonal matrix

$$
\mathcal{C}=\left(\begin{array}{ccccc}
P_{-m_{2}}^{2} & -\frac{P_{-m_{2}} P_{-m_{2}+1}}{2} & 0 & \cdots & 0 \\
-\frac{P_{-m_{2}} P_{-m_{2}+1}}{2} & P_{-m_{2}+1}^{2} & -\frac{P_{-m_{2}+1} P_{-m_{2}+2}}{2} & \cdots & 0 \\
0 & -\frac{P_{-m_{2}+1} P_{-m_{2}+2}}{2} & P_{-m_{2}+2}^{2} & \cdots & 0 \\
\vdots & \vdots & \vdots & \ddots & \vdots \\
0 & 0 & 0 & \cdots & P_{m_{1}}^{2}
\end{array}\right)
$$

This matrix satisfies all the assumptions of Lemma 1.2 on the compact sets $E_{j}=\operatorname{supp}\left(\sigma_{j}^{1}\right), j=$ $0,1, \ldots, m_{1}, E_{j}=\operatorname{supp}\left(\sigma_{-j}^{2}\right), j=0,-1, \ldots,-m_{2}$, including $c_{j, k} \geq 0$ when $E_{j} \cap E_{k} \neq \emptyset$ (recall that $\left.\sigma_{0}^{1}=\sigma_{0}^{2}\right)$, and it is positive definite because the principal section $\mathcal{C}_{r}, r=1, \ldots, m_{1}+m_{2}+1$, of $\mathcal{C}$ satisfies

$$
\operatorname{det}\left(\mathcal{C}_{r}\right)=P_{-m_{2}}^{2} \cdots P_{-m_{2}+r-1}^{2} \operatorname{det}\left(\begin{array}{cccccc}
1 & -\frac{1}{2} & 0 & \cdots & 0 & 0 \\
-\frac{1}{2} & 1 & -\frac{1}{2} & \cdots & 0 & 0 \\
0 & -\frac{1}{2} & 1 & \cdots & 0 & 0 \\
\vdots & \vdots & \vdots & \ddots & \vdots & \vdots \\
0 & 0 & 0 & \cdots & 1 & -\frac{1}{2} \\
0 & 0 & 0 & \cdots & -\frac{1}{2} & 1
\end{array}\right)_{r \times r}>0
$$

Let $\bar{\mu}(\mathcal{C})$ be the equilibrium solution for the corresponding vector potential problem. We have

Theorem 1.3. Let $\Lambda=\Lambda\left(p_{1,0}, \ldots, p_{1, m_{1}} ; p_{2,0}, \ldots, p_{2, m_{2}}\right) \subset \mathbb{Z}_{+}^{m_{1}+1}(\bullet) \times \mathbb{Z}_{+}^{m_{2}+1}(\bullet),\left(S^{1}, S^{2}\right) \in \mathbf{R e g}$, $S^{1}=\mathcal{N}\left(\sigma_{0}^{1}, \ldots, \sigma_{m_{1}}^{1}\right)$, and $S^{2}=\mathcal{N}\left(\sigma_{0}^{2}, \ldots, \sigma_{m_{2}}^{2}\right)$ be given. Then

$$
\lim _{\mathbf{n} \in \Lambda}\left|\mathcal{A}_{\mathbf{n}, 0}(z)\right|^{1 /\left|\mathbf{n}_{1}\right|}=G_{0}(z), \quad \mathcal{K} \subset \mathbb{C} \backslash\left(\Delta_{0}^{1} \cup \Delta_{1}^{1}\right),
$$

uniformly on each compact subset $\mathcal{K} \subset \mathbb{C} \backslash\left(\Delta_{0}^{1} \cup \Delta_{1}^{1}\right)$, where

$$
G_{0}(z)=\exp \left(P_{1} V^{\bar{\mu}_{1}}(z)-V^{\bar{\mu}_{0}}(z)-2 \sum_{k=1}^{m_{1}} \frac{\omega_{k}^{\bar{\mu}}}{P_{k}}\right) .
$$

$\bar{\mu}=\bar{\mu}(\mathcal{C})=\left(\bar{\mu}_{-m_{2}}, \ldots, \bar{\mu}_{m_{1}}\right)$ is the equilibrium vector measure and $\left(\omega_{-m_{2}}^{\bar{\mu}}, \ldots, \omega_{m_{1}}^{\bar{\mu}}\right)$ is the system of equilibrium constants for the vector potential problem determined by the interaction matrix $\mathcal{C}$ defined in (4) on the system of compact sets $E_{j}=\operatorname{supp}\left(\sigma_{j}^{1}\right), j=0, \ldots, m_{1}, E_{j}=\operatorname{supp}\left(\sigma_{-j}^{2}\right), j=$ $-m_{2}, \ldots, 0$.

Throughout the paper, the notation

$$
\lim _{n \in \Lambda} g_{n}(z)=g(z), \quad \mathcal{K} \subset \Omega,
$$

stands for uniform convergence of the sequence $\left\{g_{n}\right\}, n \in \Lambda$, to $g$ on each compact subset $\mathcal{K}$ contained in the indicated region (in this case $\Omega$ ). 
For the next result, we assume that $\operatorname{supp}\left(\sigma_{j}^{i}\right)=\widetilde{\Delta}_{j}^{i} \cup e_{j}^{i}, i=1,2$, where $\widetilde{\Delta}_{j}^{i}$ is a bounded interval of the real line, $\left|\left(\sigma_{j}^{i}\right)^{\prime}\right|>0$ a.e. on $\widetilde{\Delta}_{j}^{i}$, and $e_{j}^{i}$ is at most a denumerable set without accumulation points in $\mathbb{R} \backslash \widetilde{\Delta}_{j}^{i}$. We denote this writing

$$
S^{1}=\mathcal{N}^{\prime}\left(\sigma_{0}^{1}, \ldots, \sigma_{m_{1}}^{1}\right), \quad S^{2}=\mathcal{N}^{\prime}\left(\sigma_{0}^{2}, \ldots, \sigma_{m_{2}}^{2}\right)
$$

In the context of this paper, this condition is the analogue of the one imposed by S. A. Denisov in his extension of Rakhmanov's theorem (see paragraph containing (1) and the one that follows it).

Fix a vector $l:=\left(l_{1} ; l_{2}\right)$ where $0 \leq l_{1} \leq m_{1}$ and $0 \leq l_{2} \leq m_{2}$. We define the multi-index $\mathbf{n}^{l}:=\left(\mathbf{n}_{1}+\mathbf{e}^{l_{1}} ; \mathbf{n}_{2}+\mathbf{e}^{l_{2}}\right)=\left(\mathbf{n}_{1}^{l_{1}} ; \mathbf{n}_{2}^{l_{2}}\right)$, where $\mathbf{e}^{l_{i}}$ denotes the unit vector of length $m_{i}+1$ with all components equal to zero except the component $\left(l_{i}+1\right)$ which equals 1 . It is always assumed that both $\mathbf{n}$ and $\mathbf{n}^{l}$ belong to $\mathbb{Z}_{+}^{m_{1}+1}(\bullet) \times \mathbb{Z}_{+}^{m_{2}+1}(\bullet)$.

Theorem 1.4. Let $S^{1}=\mathcal{N}^{\prime}\left(\sigma_{0}^{1}, \ldots, \sigma_{m_{1}}^{1}\right), S^{2}=\mathcal{N}^{\prime}\left(\sigma_{0}^{2}, \ldots, \sigma_{m_{2}}^{2}\right)$, and $\Lambda \subset \mathbb{Z}_{+}^{m_{1}+1}(\bullet) \times \mathbb{Z}_{+}^{m_{2}+1}(\bullet)$ is an infinite sequence of distinct multi-indices such that

$$
\sup _{\mathbf{n} \in \Lambda}\left(n_{1,0}-n_{1, m_{1}}\right)<\infty, \quad \sup _{\mathbf{n} \in \Lambda}\left(n_{2,0}-n_{1, m_{2}}\right)<\infty .
$$

Assume that there exists $l=\left(l_{1} ; l_{2}\right), 0 \leq l_{1} \leq m_{1}, 0 \leq l_{2} \leq m_{2}$, such that for all $\mathbf{n} \in \Lambda$ we have that $\mathbf{n}^{l}=\left(\mathbf{n}_{1}^{l_{1}} ; \mathbf{n}_{2}^{l_{2}}\right) \in \mathbb{Z}_{+}^{m_{1}+1}(\bullet) \times \mathbb{Z}_{+}^{m_{2}+1}(\bullet)$. Then

$$
\lim _{\mathbf{n} \in \Lambda} \frac{\mathcal{A}_{\mathbf{n}^{l}, 0}(z)}{\mathcal{A}_{\mathbf{n}, 0}(z)}=\mathcal{A}_{0}^{(l)}(z), \quad \mathcal{K} \subset \mathbb{C} \backslash\left(\operatorname{supp}\left(\sigma_{0}^{1}\right) \cup \operatorname{supp}\left(\sigma_{1}^{1}\right)\right),
$$

where $\mathcal{A}_{0}^{(l)}(z)$ is a one to one analytic function in $\mathbb{C} \backslash\left(\widetilde{\Delta}_{0}^{1} \cup \widetilde{\Delta}_{1}^{1}\right)$.

An expression for $\mathcal{A}_{0}^{(l)}(z)$ will be given in (96) (see also Theorem 6.8). The answer depends on the conformal representation of an associated Riemann surface with $m_{1}+m_{2}+2$ sheets and genus zero onto the extended complex plane. The previous result is already new when $m_{2}=0$ and $m_{1} \geq 2$. The assumption (6) means that the components of the multi-indices $\mathbf{n}_{1}$ and $\mathbf{n}_{2}$ are nearly equal. The need for this requirement is explained in the second last paragraph of the proof of Lemma 6.1.

Besides normality, in Section 2 we obtain the orthogonality relations satisfied by the linear forms involved in the construction. Section 4 is devoted to the study of the asymptotic distribution of zeros of a system of linear forms associated with $\mathcal{A}_{\mathbf{n}, 0}$ that allows to prove Theorem 5.1 in Section 5 of which Theorem 1.3 is a corollary. Theorem 5.1 was first stated in [24] under the stronger assumptions $S^{1}=\mathcal{N}^{\prime}\left(\sigma_{0}^{1}, \ldots, \sigma_{m_{1}}^{1}\right), S^{2}=\mathcal{N}^{\prime}\left(\sigma_{0}^{2}, \ldots, \sigma_{m_{2}}^{2}\right)$, and $\operatorname{supp}\left(\sigma_{j}^{i}\right)=\widetilde{\Delta}_{j}^{i}, i=1,2$; and the proof was carried out only when $m_{1}=m_{2}=1$.

In Section 3 we study the interlacing properties of the zeros of the linear forms which is needed for the proof of Theorem 6.8 in Section 6 from which Theorem 1.4 follows. Section 7 contains a Markov type theorem for mixed type Hermite-Padé approximation and some reinterpretation of 
the theory developed in the context of systems of bi-orthogonal linear forms. All the results of the paper are enumerated consecutively by sections.

\section{NORMALity AND ORTHOGONALITY RELATIONS}

Recall that

$$
s_{j, k}=\left\langle\sigma_{j}, \ldots, \sigma_{k}\right\rangle, \quad 0 \leq j \leq k \leq m, \quad s_{j, j}=\sigma_{j} .
$$

We denote $\left(\widehat{s}_{j+1, j}^{1}(z) \equiv 1, \mathcal{A}_{\mathbf{n}, m_{1}} \equiv a_{\mathbf{n}, m_{1}}\right)$

$$
\mathcal{A}_{\mathbf{n}, j}(z):=\sum_{k=j}^{m_{1}} a_{\mathbf{n}, k}(z) \widehat{s}_{j+1, k}^{1}(z), \quad j=0, \ldots, m_{1} .
$$

In [18], E. M. Nikishin introduced the following definition.

Definition 2.1. A set of real continuous functions $u_{0}(x), \ldots, u_{m_{1}}(x)$ defined on an interval $\Delta$, is called an AT-system for $\mathbf{n}_{1}=\left(n_{1,0}, \ldots, n_{1, m_{1}}\right) \in \mathbb{Z}_{+}^{m_{1}+1}$, if for any polynomials $h_{0} \ldots, h_{m_{1}}$ such that $\operatorname{deg}\left(h_{i}\right) \leq n_{1, i}-1, i=0, \ldots, m_{1}$, not simultaneously identically equal to zero, the function

$$
h_{0}(x) u_{0}(x)+\cdots+h_{m_{1}}(x) u_{m_{1}}(x),
$$

has at most $\left|\mathbf{n}_{1}\right|-1$ zeros on $\Delta\left(\operatorname{deg}\left(h_{j}\right) \leq-1\right.$ means that $\left.h_{j} \equiv 0\right)$.

Let $\mathbb{Z}_{+}^{m_{1}+1}(*)$ be the set of multi-indices given by

$$
\mathbb{Z}_{+}^{m_{1}+1}(*)=\left\{\mathbf{n}_{1} \in \mathbb{Z}_{+}^{m_{1}+1}: \nexists i<k<j \text { such that } n_{i}<n_{j}<n_{k}\right\} .
$$

Obviously, this class of multi-indices contains $\mathbb{Z}_{+}^{m_{1}+1}(\bullet)$. In connection with AT-systems, in [8] U. Fidalgo and G. López proved

Lemma 2.2. Let $\mathbf{n}_{1} \in \mathbb{Z}_{+}^{m_{1}+1}(*)$ and $\left(s_{1}, \ldots, s_{m_{1}}\right)=\mathcal{N}\left(\sigma_{1}, \ldots, \sigma_{m_{1}}\right)$, then the system of functions $\left(1, \widehat{s}_{1}, \ldots, \widehat{s}_{m_{1}}\right)$ defines an AT-system with respect to $\mathbf{n}_{1}=\left(n_{1,0}, \ldots, n_{1, m_{1}}\right)$ on any interval disjoint from $\operatorname{Co}\left(\operatorname{supp}\left(\sigma_{1}\right)\right)$.

For each $j=0, \ldots, m_{1}-1$, we have that $\left(s_{j+1, j+1}^{1}, \ldots, s_{j+1, m_{1}}^{1}\right)=\mathcal{N}\left(\sigma_{j+1}^{1}, \ldots, \sigma_{m_{1}}^{1}\right)$. Using Lemma 2.2 it follows that for $\mathbf{n}_{1} \in \mathbb{Z}_{+}^{m_{1}+1}(\bullet) \subset \mathbb{Z}_{+}^{m_{1}+1}(*)$ the linear form $\mathcal{A}_{\mathbf{n}, j}$ cannot have more than $N_{1, j}-1$ zeros on any interval disjoint from $\Delta_{j+1}^{1}$, where

$$
N_{1, j}=N_{1, j}(\mathbf{n})=n_{1, j}+\cdots+n_{1, m_{1}} .
$$

Obviously, the same is true for the polynomial $\mathcal{A}_{\mathbf{n}, m_{1}} \equiv a_{\mathbf{n}, m_{1}}$. (Below, we also use the previous lemma for linear forms generated by the second Nikishin system.)

Let us prove that $\mathcal{A}_{\mathbf{n}, 0}$ has at least $\left|\mathbf{n}_{1}\right|-1=\left|\mathbf{n}_{2}\right|$ sign changes on the interval $\Delta_{0}^{1}=\Delta_{0}^{2}$.

Notice that $d s_{0, k}^{2}(x)=\widehat{s}_{1, k}^{2}(x) d \sigma_{0}^{2}(x)$. On the other hand, we can replace $x^{\nu}$ by any polynomial of degree $\leq n_{2, k}-1$ inside the integral in (2). Set

$$
\mathcal{B}_{\mathbf{n}_{2}}(z)=\sum_{k=0}^{m_{2}} b_{\mathbf{n}_{2}, k}(z) \widehat{s}_{1, k}^{2}(z), \quad \operatorname{deg} b_{\mathbf{n}_{2}, k} \leq n_{2, k}-1, \quad k=0, \ldots, m_{2} .
$$


$\left(\widehat{s}_{1,0}^{2}(z) \equiv 1\right)$. Then $(2)$ is equivalent to

$$
\int \mathcal{B}_{\mathbf{n}_{2}}(x) \mathcal{A}_{\mathbf{n}, 0}(x) d \sigma_{0}^{2}(x)=0,
$$

for all $\mathcal{B}_{\mathbf{n}_{2}}$ as indicated.

Suppose that $\mathcal{A}_{\mathbf{n}, 0}$ has at most $N<\left|\mathbf{n}_{1}\right|-1=\left|\mathbf{n}_{2}\right|$ sign changes on the interval $\Delta_{0}^{2}$. Choose polynomials $b_{\mathbf{n}_{2}, k}$ conveniently so that $\mathcal{B}_{\mathbf{n}_{2}}$ changes sign exactly at those points where $\mathcal{A}_{\mathbf{n}, 0}$ changes sign on $\Delta_{0}^{2}$ and has a zero of order $\left|\mathbf{n}_{2}\right|-1-N$ at one of the extreme points of $\Delta_{0}^{1}=\Delta_{0}^{2}$. By Lemma 2.2, the linear form $\mathcal{B}_{\mathbf{n}_{2}}$ has on $\Delta_{0}^{2}$ at most $\left|\mathbf{n}_{2}\right|-1$ zeros, thus it can only have those zeros which we have assigned to it. The continuous function $\mathcal{B}_{\mathbf{n}_{2}} \mathcal{A}_{\mathbf{n}, 0}$ has constant sign on $\Delta_{0}^{2}$. This contradicts (8).

We have proved that $\mathcal{A}_{\mathbf{n}, 0}$ has $\left|\mathbf{n}_{1}\right|-1$ zeros with odd multiplicity in the interior of $\Delta_{0}^{2}=\Delta_{0}^{1}$. In connection with intervals of the real line, the interior refers to the Euclidean topology of $\mathbb{R}$. In short, we shall see that $\mathcal{A}_{\mathbf{n}, 0}$ has no other zeros in $\mathbb{C} \backslash \Delta_{1}^{1}$ and that they are all simple. Before proving this, let us turn to the question of normality. The concept of normal multi-index was introduced in Definition 1.1.

Proposition 2.3. Let $\mathbf{n} \in \mathbb{Z}_{+}^{m_{1}+1}(\bullet) \times \mathbb{Z}_{+}^{m_{2}+1}(\bullet), S^{1}=\mathcal{N}\left(\sigma_{0}^{1}, \ldots, \sigma_{m_{1}}^{1}\right)$, and $S^{2}=\mathcal{N}\left(\sigma_{0}^{2}, \ldots, \sigma_{m_{2}}^{2}\right)$, be given. Then, $\mathbf{n}$ is normal and $\left(a_{\mathbf{n}, 0}, \ldots, a_{\mathbf{n}, m_{1}}\right)$ is uniquely determined except for a constant factor.

Proof. Assume that there exists $j \in\left\{0, \ldots, m_{1}\right\}$ such that $\operatorname{deg} a_{\mathbf{n}, j} \leq n_{1, j}-2$. Then $\mathbf{n}_{1}-\mathbf{e}^{j} \in$ $\mathbb{Z}_{+}^{m_{1}+1}(*)$, where $\mathbf{e}^{j}$ denotes the $m_{1}+1$ dimensional unit vector with all components equal to zero except the component $j+1$ which equals 1 . According to Lemma 2.2 applied to $\mathbf{n}_{1}-\mathbf{e}^{j}$, the linear form $\mathcal{A}_{\mathbf{n}, 0}$ has at most $\left|\mathbf{n}_{1}\right|-2$ zeros on $\Delta_{0}^{1}$, but we pointed out before that it has at least $\left|\mathbf{n}_{1}\right|-1$ sign changes on this interval. This contradiction yields that for all $j \in\left\{0, \ldots, m_{1}\right\}$, $\operatorname{deg} a_{\mathbf{n}, j}=n_{1, j}-1$, which implies normality.

Now, let us assume that $\left(a_{\mathbf{n}, 0}, \ldots, a_{\mathbf{n}, m_{1}}\right)$ and $\left(a_{\mathbf{n}, 0}^{*}, \ldots, a_{\mathbf{n}, m_{1}}^{*}\right)$ solve i')-ii') and these vectors are not collinear. According to what we just proved, for all $j \in\left\{0, \ldots, m_{1}\right\}, \operatorname{deg} a_{\mathbf{n}, j}=\operatorname{deg} a_{\mathbf{n}, j}^{*}=$ $n_{1, j}-1$. Take $\lambda \in \mathbb{C} \backslash\{0\}$ such that $\operatorname{deg}\left(a_{\mathbf{n}, 0}-\lambda a_{\mathbf{n}, 0}^{*}\right) \leq n_{1,0}-2$. Obviously, the vector $\left(a_{\mathbf{n}, 0}-\right.$ $\left.\lambda a_{\mathbf{n}, 0}^{*}, \ldots, a_{\mathbf{n}, m_{1}}-\lambda a_{\mathbf{n}, m_{1}}^{*}\right)$ is not identically equal to zero and also solves i')-ii') which is not possible since all non trivial solutions must have all components of maximal degree.

Proposition 2.3 allows us to determine the "monic" $\left(a_{\mathbf{n}, 0}, a_{\mathbf{n}, 1}, \ldots, a_{\mathbf{n}, m_{1}}\right)$ uniquely and we impose this normalization (see Definition 1.1). The next lemma will be used on several occasions.

Lemma 2.4. Let $s_{k}, k=1, \ldots, m$, be finite signed Borel measures, $\operatorname{Co}\left(\operatorname{supp}\left(s_{k}\right)\right)=\Delta \subset \mathbb{R}$. Let $F(z)=f_{0}(z)+\sum_{k=1}^{m} f_{k}(z) \widehat{s_{k}}(z) \in \mathcal{H}(\overline{\mathbb{C}} \backslash \Delta)$, where $f_{k} \in \mathcal{H}(V), k=0, \ldots, m$, and $V$ is a 
neighborhood of $\Delta$. If $F(z)=\mathcal{O}\left(1 / z^{2}\right), z \rightarrow \infty$, then

$$
\sum_{k=1}^{m} \int f_{k}(x) d s_{k}(x)=0
$$

and $F(z)=\mathcal{O}(1 / z), z \rightarrow \infty$, implies that

$$
F(z)=\sum_{k=1}^{m} \int \frac{f_{k}(x) d s_{k}(x)}{z-x}
$$

Proof. Let $\Gamma \subset V$ be a closed smooth Jordan curve that surrounds $\Delta$. If $F(z)=\mathcal{O}\left(1 / z^{2}\right), z \rightarrow \infty$, from Cauchy's theorem, Fubini's theorem and Cauchy's integral formula, it follows that

$$
0=\int_{\Gamma} F(z) d z=\sum_{k=1}^{m} \int_{\Gamma} f_{k}(z) \widehat{s_{k}}(z) d z=\sum_{k=1}^{m} \iint_{\Gamma} \frac{f_{k}(z) d z}{z-x} d s_{k}(x)=2 \pi i \sum_{k=1}^{m} \int f_{k}(x) d s_{k}(x),
$$

and we obtain (9). On the other hand, if $F(z)=\mathcal{O}(1 / z), z \rightarrow \infty$, and we assume that $z$ is in the unbouded connected component of the complement of $\Gamma$, Cauchy's integral formula and Fubini's theorem render

$$
\begin{gathered}
F(z)=\frac{1}{2 \pi i} \int_{\Gamma} \frac{F(\zeta) d \zeta}{z-\zeta}=\frac{1}{2 \pi i} \sum_{k=1}^{m} \int_{\Gamma} \frac{f_{k}(\zeta) \widehat{s}_{k}(\zeta) d \zeta}{z-\zeta}= \\
\sum_{k=1}^{m} \int \frac{1}{2 \pi i} \int_{\Gamma} \frac{f_{k}(\zeta) d \zeta}{(z-\zeta)(\zeta-x)} d s_{k}(x)=\sum_{k=1}^{m} \int \frac{f_{k}(x) d s_{k}(x)}{z-x}
\end{gathered}
$$

which is (10).

In the sequel, $\mathbf{n}=\left(\mathbf{n}_{1} ; \mathbf{n}_{2}\right) \in \mathbb{Z}_{+}^{m_{1}+1}(\bullet) \times \mathbb{Z}_{+}^{m_{2}+1}(\bullet)$ and $n_{1, m_{1}} \geq 1$. For $j=0, \ldots, m_{1}$, let $Q_{\mathbf{n}, j}$ be the monic polynomial whose zeros are those of the linear form $\mathcal{A}_{\mathbf{n}, j}$ in the region $\mathbb{C} \backslash \Delta_{j+1}^{1}$, counting multiplicities $\left(\Delta_{m_{1}+1}^{1}=\emptyset\right)$. In particular, $\mathcal{A}_{\mathbf{n}, m_{1}}=a_{\mathbf{n}, m_{1}}=Q_{\mathbf{n}, m_{1}}$. From the previous proposition, if $n_{1, m_{1}} \geq 1, \infty$ is not a zero of any one of these linear forms; thus, $\infty$ cannot be an accumulation point of such zeros. Though it is not the case, in principle, some of these linear forms may have an infinite number of zeros which accumulate on the boundary of the corresponding region of meromorphy. In that case, for the time being, $Q_{\mathbf{n}, j}$ denotes a formal infinite product.

The next proposition is adapted from [15].

Proposition 2.5. Let $\mathbf{n} \in \mathbb{Z}_{+}^{m_{1}+1}(\bullet) \times \mathbb{Z}_{+}^{m_{2}+1}(\bullet), n_{1, m_{1}} \geq 1, S^{1}=\mathcal{N}\left(\sigma_{0}^{1}, \ldots, \sigma_{m_{1}}^{1}\right)$, and $S^{2}=$ $\mathcal{N}\left(\sigma_{0}^{2}, \ldots, \sigma_{m_{2}}^{2}\right)$ be given (recall that $\left.\sigma_{0}^{1}=\sigma_{0}^{2}\right)$. Then, $\operatorname{deg} Q_{\mathbf{n}, j}=N_{1, j}-1, j=0,1 \ldots, m_{1}$, all its zeros are simple and lie in the interior of $\Delta_{j}^{1}$. If I denotes the closure of a connected component of $\Delta_{j}^{1} \backslash \operatorname{supp}\left(\sigma_{j}^{1}\right)$ then $Q_{\mathbf{n}, j}$ has at most one zero in I. Moreover,

$$
\int x^{\nu} \mathcal{A}_{\mathbf{n}, j}(x) \frac{d \sigma_{j}^{1}(x)}{Q_{\mathbf{n}, j-1}(x)}=0, \quad \nu=0, \ldots, N_{1, j}-2, \quad j=1, \ldots, m_{1},
$$

and for any polynomial $q, \operatorname{deg} q \leq N_{1, j+1}-1$,

$$
\frac{q(z) \mathcal{A}_{\mathbf{n}, j}(z)}{Q_{\mathbf{n}, j}(z)}=\int \frac{q(x) \mathcal{A}_{\mathbf{n}, j+1}(x)}{Q_{\mathbf{n}, j}(x)} \frac{d \sigma_{j+1}^{1}(x)}{z-x}, \quad j=0, \ldots, m_{1}-1 .
$$


Proof. Using induction on $j$, we will prove simultaneously the general statement concerning the zeros and (11). Then, we prove that on any interval $I$ there is at most one zero of $Q_{\mathbf{n}, j}$. Finally, we obtain (12). For $j=0$, we already proved that $\mathcal{A}_{\mathbf{n}, 0}$ has $N_{1,0}-1=\left|\mathbf{n}_{1}\right|-1$ sign changes in the interior of $\Delta_{0}^{1}=\Delta_{0}^{2}$. Therefore, $\operatorname{deg} Q_{\mathbf{n}, 0} \geq N_{1,0}-1$. If $\operatorname{deg} Q_{\mathbf{n}, 0}=N_{1,0}-1$ we conclude with the initial step.

Suppose that $\operatorname{deg} Q_{\mathbf{n}, 0} \geq N_{1,0}$ (including the possible case that $\operatorname{deg} Q_{\mathbf{n}, 0}=\infty$ ). It is easy to see that $\mathcal{A}_{\mathbf{n}, 0}(\bar{z})=\overline{\mathcal{A}_{\mathbf{n}, 0}(z)}$, so the zeros of $Q_{\mathbf{n}, 0}$ come in conjugate pairs. Therefore, we can choose $N_{1,0}$ (or $N_{1,0}+1$ if necessary) zeros of $Q_{\mathbf{n}, 0}$ in such a way that the monic polynomial $Q_{\mathbf{n}, 0}^{*}$ with this set of zeros has constant sign on $\Delta_{1}^{1}\left(\Delta_{1}^{1} \cap \Delta_{0}^{1}=\emptyset\right)$. Notice that

$$
\frac{\mathcal{A}_{\mathbf{n}, 0}}{Q_{\mathbf{n}, 0}^{*}} \in \mathcal{H}\left(\overline{\mathbb{C}} \backslash \Delta_{1}^{1}\right)
$$

is analytic in the indicated region and

$$
\frac{z^{\nu} \mathcal{A}_{\mathbf{n}, 0}}{Q_{\mathbf{n}, 0}^{*}}=\mathcal{O}\left(\frac{1}{z^{2}}\right), \quad \nu=0, \ldots, N_{1,1}-1
$$

In this paper the symbol $\mathcal{O}(\cdot)$ always refers to $z \rightarrow \infty$. From (9), we get

$$
0=\int x^{\nu} \mathcal{A}_{\mathbf{n}, 1}(x) \frac{d \sigma_{1}^{1}(x)}{Q_{\mathbf{n}, 0}^{*}(x)}, \quad \nu=0, \ldots, N_{1,1}-1 .
$$

This implies that $\mathcal{A}_{\mathbf{n}, 1}$ has at least $N_{1,1}$ zeros on $\Delta_{1}^{1}$. According to Lemma 2.2 this linear form can only have $N_{1,1}-1$ zeros on this interval. Consequently, our initial assumption is false and $\operatorname{deg} Q_{\mathbf{n}, 0}=N_{1,0}-1$.

Suppose that we have proved that for some $j \in\left\{0, \ldots, m_{1}-1\right\}, \operatorname{deg} Q_{\mathbf{n}, j}=N_{1, j}-1$, all its zeros are simple and lie in the interior of $\Delta_{j}^{1}$. Let us show that then, (11) and the statement concerning the zeros are valid for $j+1$.

Indeed, the induction hypothesis implies that

$$
\frac{\mathcal{A}_{\mathbf{n}, j}}{Q_{\mathbf{n}, j}} \in \mathcal{H}\left(\overline{\mathbb{C}} \backslash \Delta_{j+1}^{1}\right), \quad \frac{z^{\nu} \mathcal{A}_{\mathbf{n}, j}}{Q_{\mathbf{n}, j}}=\mathcal{O}\left(\frac{1}{z^{2}}\right), \quad \nu=0, \ldots, N_{1, j+1}-2 .
$$

From (9), it follows that

$$
0=\int x^{\nu} \mathcal{A}_{\mathbf{n}, j+1}(x) \frac{d \sigma_{j+1}^{1}(x)}{Q_{\mathbf{n}, j}(x)}, \quad \nu=0, \ldots, N_{1, j+1}-2 .
$$

We have obtained (11) for $j+1$.

Formula (11) for $j+1$ implies that $Q_{\mathbf{n}, j+1}$ has at least $N_{1, j+1}-1$ sign changes in the interior of $\Delta_{j+1}^{1}$. If $\operatorname{deg} Q_{\mathbf{n}, j+1}=N_{1, j+1}-1$, we have finished the proof (for example, this is the case when $j+1=m_{1}$ because $\left.\mathcal{A}_{\mathbf{n}, m_{1}} \equiv a_{\mathbf{n}, m_{1}}\right)$. Let us suppose that $\operatorname{deg} Q_{\mathbf{n}, j+1} \geq N_{1, j+1}$ (including the possible case that $\operatorname{deg} Q_{\mathbf{n}, j+1}=\infty$, and of course $\left.j \leq m_{1}-2\right)$. Since $\mathcal{A}_{\mathbf{n}, j+1}(\bar{z})=\overline{\mathcal{A}_{\mathbf{n}, j+1}(z)}$, we can choose $N_{1, j+1}$ (or $N_{1, j+1}+1$ if necessary) zeros of $Q_{\mathbf{n}, j+1}$ so that the monic polynomial $Q_{\mathbf{n}, j+1}^{*}$ with this set of zeros has constant sign on $\Delta_{j+2}^{1}$. Then

$$
\frac{\mathcal{A}_{\mathbf{n}, j+1}}{Q_{\mathbf{n}, j+1}^{*}} \in \mathcal{H}\left(\overline{\mathbb{C}} \backslash \Delta_{j+2}^{1}\right), \quad \frac{z^{\nu} \mathcal{A}_{\mathbf{n}, j+1}}{Q_{\mathbf{n}, j+1}^{*}}=\mathcal{O}\left(\frac{1}{z^{2}}\right), \quad \nu=0, \ldots, N_{1, j+2}-1
$$


Using (9), it follows that

$$
0=\int x^{\nu} \mathcal{A}_{\mathbf{n}, j+2}(x) \frac{d \sigma_{j+2}^{1}(x)}{Q_{\mathbf{n}, j+1}^{*}(x)}, \quad \nu=0, \ldots, N_{1, j+2}-1 .
$$

This implies that $\mathcal{A}_{\mathbf{n}, j+2}$ has at least $N_{1, j+2}$ zeros on $\Delta_{j+2}^{1}$. According to Lemma 2.2 this linear form can only have $N_{1, j+2}-1$ zeros on this interval. This implies that our initial assumption is false; therefore, $\operatorname{deg} Q_{\mathbf{n}, j+1}=N_{1, j+1}-1$ as stated.

Suppose that the interval $I$ contains two zeros $x_{1}, x_{2}$ of $Q_{\mathbf{n}, j}$; that is, of $\mathcal{A}_{\mathbf{n}, j}$. According to (11)

$$
\int x^{\nu} \frac{\mathcal{A}_{\mathbf{n}, j}(x)}{\left(x-x_{1}\right)\left(x-x_{2}\right)} \frac{\left(x-x_{1}\right)\left(x-x_{2}\right) d \sigma_{j}^{1}(x)}{Q_{\mathbf{n}, j-1}(x)}=0, \quad \nu=0, \ldots, N_{1, j}-2 .
$$

The function $\mathcal{A}_{\mathbf{n}, j}(x) /\left(x-x_{1}\right)\left(x-x_{2}\right)$ has $N_{1, j}-3$ sign changes on $\operatorname{supp}\left(\sigma_{j}^{1}\right)$ while the measure $\left(x-x_{1}\right)\left(x-x_{2}\right) d \sigma_{j}^{1}(x) / Q_{\mathbf{n}, j-1}(x)$ has constant sign on $\operatorname{supp}\left(\sigma_{j}^{1}\right)$. This is impossible because of the number of orthogonality relations.

Formula (12) follows from (10) since for any $q, \operatorname{deg} q \leq N_{1, j+1}-1$,

$$
\frac{q \mathcal{A}_{\mathbf{n}, j}}{Q_{\mathbf{n}, j}} \in \mathcal{H}\left(\overline{\mathbb{C}} \backslash \Delta_{j+1}^{1}\right), \quad \frac{q \mathcal{A}_{\mathbf{n}, j}}{Q_{\mathbf{n}, j}}=\mathcal{O}\left(\frac{1}{z}\right) .
$$

With this we conclude the proof.

We need to produce additional orthogonality relations. In the second part of this section, we make use of some transformations employed in [11]. Let us define recursively the following functions

$$
\mathcal{A}_{\mathbf{n},-j-1}(z)=\int \frac{\mathcal{A}_{\mathbf{n},-j}(x)}{z-x} d \sigma_{j}^{2}(x), \quad j=0, \ldots, m_{2} .
$$

Proposition 2.6. Let $\mathbf{n} \in \mathbb{Z}_{+}^{m_{1}+1}(\bullet) \times \mathbb{Z}_{+}^{m_{2}+1}(\bullet), n_{1, m_{1}} \geq 1, S^{1}=\mathcal{N}\left(\sigma_{0}^{1}, \ldots, \sigma_{m_{1}}^{1}\right)$, and $S^{2}=$ $\mathcal{N}\left(\sigma_{0}^{2}, \ldots, \sigma_{m_{2}}^{2}\right)$ be given. Then, for each $j=0, \ldots, m_{2}$

$$
\int x^{\nu} \mathcal{A}_{\mathbf{n},-j}(x) d s_{j, k}^{2}(x)=0, \quad k=j, \ldots, m_{2}, \quad \nu=0, \ldots, n_{2, k}-1 .
$$

Proof. When $j=0$ the statement reduces to the relations ii) which define $\mathcal{A}_{\mathbf{n}, 0}$. If $m_{2}=0$ we are done. Therefore, let us assume that $m_{2} \geq 1$, that (14) holds for some $j \in\left\{0, \ldots, m_{2}-1\right\}$, and prove that it is also satisfied for $j+1$.

Fix $j \in\left\{0, \ldots, m_{2}-1\right\}, k \in\left\{j+1, \ldots, m_{2}\right\}$, and $\nu \in\left\{0, \ldots, n_{2, k}-1\right\}$. Using the definition of $\mathcal{A}_{\mathbf{n},-j-1}$, Fubini's theorem, and the induction hypothesis, we obtain

$$
\begin{gathered}
\int x^{\nu} \mathcal{A}_{\mathbf{n},-j-1}(x) d s_{j+1, k}^{2}(x)=\int x^{\nu} \int \frac{\mathcal{A}_{\mathbf{n},-j}(t)}{x-t} d \sigma_{j}^{2}(t) d s_{j+1, k}^{2}(x)= \\
\int \mathcal{A}_{\mathbf{n},-j}(t) \int \frac{x^{\nu} \mp t^{\nu}}{x-t} d s_{j+1, k}^{2}(x) d \sigma_{j}^{2}(t)= \\
\int p_{\nu}(t) \mathcal{A}_{\mathbf{n},-j}(t) d \sigma_{j}^{2}(t)-\int t^{\nu} \mathcal{A}_{\mathbf{n},-j}(t) d s_{j, k}^{2}(t)=0
\end{gathered}
$$

since $p_{\nu}$ is a polynomial of degree $\leq n_{2, k}-2$, and $n_{2, j+1} \geq n_{2, k}$. 
For $j=1, \ldots, m_{2}+1$, let $Q_{\mathbf{n},-j}$ be the monic polynomial whose zeros are those of $\mathcal{A}_{\mathbf{n},-j}$ in the region $\mathbb{C} \backslash \Delta_{j-1}^{2}$ counting multiplicities. As we did before, in the hypothetical case that $\mathcal{A}_{\mathbf{n},-j}$ had infinitely many zeros in the specified region, then $Q_{\mathbf{n},-j}$ denotes a formal infinite product.

Taking linear combinations of the relations (14), we obtain

$$
\int \mathcal{B}_{\mathbf{n}_{2}, j}(x) \mathcal{A}_{\mathbf{n},-j}(x) d \sigma_{j}^{2}(x)=0, \quad j=0, \ldots, m_{2},
$$

where $\mathcal{B}_{\mathbf{n}_{2}, j}$ is an arbitrary linear form of type

$$
\mathcal{B}_{\mathbf{n}_{2}, j}(x)=\sum_{k=j}^{m_{2}} b_{k}(x) \widehat{s}_{j+1, k}^{2}(x), \quad \operatorname{deg} b_{k} \leq n_{2, k}-1 .
$$

Using Lemma 2.2, it follows that $\mathcal{A}_{\mathbf{n},-j}$ has at least $N_{2, j}$ sign changes on $\Delta_{j}^{2}$, where

$$
N_{2, j}=N_{2, j}(\mathbf{n})=n_{2, j}+\cdots+n_{2, m_{2}}, \quad j=1, \ldots, m_{2} .
$$

Consequently, $\operatorname{deg} Q_{n,-j} \geq N_{2, j}, j=0, \ldots, m_{2}$. Recall that for $j=0$ we proved in Proposition 2.5 that $\operatorname{deg} Q_{n, 0}=N_{2,0}=\left|\mathbf{n}_{2}\right|=\left|\mathbf{n}_{1}\right|-1$, that its zeros are simple, and lie in the interior of $\Delta_{0}^{2}=\Delta_{0}^{1}$.

Proposition 2.7. Let $\mathbf{n} \in \mathbb{Z}_{+}^{m_{1}+1}(\bullet) \times \mathbb{Z}_{+}^{m_{2}+1}(\bullet), n_{1, m_{1}} \geq 1, S^{1}=\mathcal{N}\left(\sigma_{0}^{1}, \ldots, \sigma_{m_{1}}^{1}\right)$, and $S^{2}=$ $\mathcal{N}\left(\sigma_{0}^{2}, \ldots, \sigma_{m_{2}}^{2}\right)$ be given. Then, $\operatorname{deg} Q_{\mathbf{n},-j}=N_{2, j}, j=0, \ldots, m_{2}$, all its zeros are simple and lie in the interior of $\Delta_{j}^{2}$, and $Q_{\mathbf{n},-m_{2}-1} \equiv 1$. If I denotes the closure of a connected component of $\Delta_{j}^{2} \backslash \operatorname{supp}\left(\sigma_{j}^{2}\right)$ then $Q_{\mathbf{n},-j}$ has at most one zero in I. Moreover,

$$
\int x^{\nu} \mathcal{A}_{\mathbf{n},-j}(x) \frac{d \sigma_{j}^{2}(x)}{Q_{\mathbf{n},-j-1}(x)}=0, \quad \nu=0, \ldots, N_{2, j}-1, \quad j=0, \ldots, m_{2},
$$

and for any polynomial $q, \operatorname{deg} q \leq N_{2, j-1}$,

$$
\frac{q(z) \mathcal{A}_{\mathbf{n},-j}(z)}{Q_{\mathbf{n},-j}(z)}=\int \frac{q(x) \mathcal{A}_{\mathbf{n},-j+1}(x)}{Q_{\mathbf{n},-j}(x)} \frac{d \sigma_{j-1}^{2}(x)}{z-x}, \quad j=1, \ldots, m_{2}+1 .
$$

Proof. Fix $j \in\left\{0, \ldots, m_{2}\right\}$. From (14) we have that for each $q, \operatorname{deg} q \leq n_{2, j}$,

$$
\int \frac{q(z)-q(x)}{z-x} \mathcal{A}_{\mathbf{n},-j}(x) d \sigma_{j}^{2}(x)=0 .
$$

It follows that

$$
\mathcal{A}_{\mathbf{n},-j-1}(z)=\frac{1}{q(z)} \int \frac{q(x)}{z-x} \mathcal{A}_{\mathbf{n},-j}(x) d \sigma_{j}^{2}(x)=\mathcal{O}\left(1 / z^{n_{2, j}+1}\right), \quad z \rightarrow \infty .
$$

We have shown that $\operatorname{deg} Q_{\mathbf{n},-j-1} \geq N_{2, j+1}\left(N_{2, m_{2}+1}=0\right)$. The zeros of $Q_{\mathbf{n},-j-1}$ come in conjugate pair since $\mathcal{A}_{\mathbf{n},-j-1}$ is also symmetric with respect to the real line. If $\operatorname{deg} Q_{\mathbf{n},-j-1}>$ $N_{2, j+1}$ take $N_{2, j+1}+1$ (or $N_{2, j+1}+2$ if necessary) zeros from $Q_{\mathbf{n},-j-1}$ so that the monic polynomial $Q_{\mathbf{n},-j-1}^{*}$ with these zeros has constant sign on $\Delta_{j}^{2}$. If $\operatorname{deg} Q_{\mathbf{n},-j-1}=N_{2, j+1}$ take $Q_{\mathbf{n},-j-1}^{*}=$ $Q_{\mathbf{n},-j-1}$.

Therefore,

$$
\frac{\mathcal{A}_{\mathbf{n},-j-1}}{Q_{\mathbf{n},-j-1}^{*}}=\mathcal{O}\left(1 / z^{n_{2, j}+\operatorname{deg} Q_{\mathbf{n},-j-1}^{*}+1}\right) \in \mathcal{H}\left(\overline{\mathbb{C}} \backslash \Delta_{j}^{2}\right),
$$


and

$$
\frac{z^{\nu} \mathcal{A}_{\mathbf{n},-j-1}}{Q_{\mathbf{n},-j-1}^{*}}=\mathcal{O}\left(1 / z^{2}\right) \in \mathcal{H}\left(\overline{\mathbb{C}} \backslash \Delta_{j}^{2}\right), \quad \nu=0, \ldots, n_{2, j}+\operatorname{deg} Q_{\mathbf{n},-j-1}^{*}-1 .
$$

Using (9), we obtain

$$
0=\int x^{\nu} \mathcal{A}_{\mathbf{n},-j}(x) \frac{d \sigma_{j}^{2}(x)}{Q_{\mathbf{n},-j-1}^{*}(x)}, \quad \nu=0, \ldots, n_{2, j}+\operatorname{deg} Q_{\mathbf{n},-j-1}^{*}-1 .
$$

This formula implies that $\mathcal{A}_{\mathbf{n},-j}$ has at least $n_{2, j}+\operatorname{deg} Q_{\mathbf{n},-j-1}^{*} \geq N_{2, j} \operatorname{sign}$ changes on $\Delta_{j}^{2}$. In particular, we have proved that if for some $j \in\left\{0, \ldots, m_{2}\right\}, \operatorname{deg} Q_{\mathbf{n},-j-1}>N_{2, j+1}$ then $\operatorname{deg} Q_{\mathbf{n},-j}>$ $N_{2, j}$. Going downwards on the index $j$ we would obtain that $\operatorname{deg} Q_{\mathbf{n}, 0}>N_{2,0}=\left|\mathbf{n}_{2}\right|=\left|\mathbf{n}_{1}\right|-1$ which is false according to Proposition 2.5. Consequently, for all $j \in\left\{0, \ldots, m_{2}\right\}, \operatorname{deg} Q_{\mathbf{n},-j-1}=N_{2, j+1}$ (in particular, $Q_{\mathbf{n},-m_{2}-1} \equiv 1$ ). Hence, $Q_{\mathbf{n},-j-1}^{*}=Q_{\mathbf{n},-j-1}$ and (15) follows. The proof that $I$ contains at most one zero of $Q_{\mathbf{n},-j}$ is the same as in Proposition 2.5 .

Now, fix $j \in\left\{1, \ldots, m_{2}+1\right\}$. Notice that for any $q, \operatorname{deg} q \leq N_{2, j-1}$,

$$
\frac{q \mathcal{A}_{\mathbf{n},-j}}{Q_{\mathbf{n},-j}} \in \mathcal{H}\left(\overline{\mathbb{C}} \backslash \Delta_{j-1}^{2}\right), \quad \frac{q \mathcal{A}_{\mathbf{n},-j}}{Q_{\mathbf{n},-j}}=\mathcal{O}\left(\frac{1}{z}\right), z \rightarrow \infty .
$$

Using (10), (16) readily follows.

\section{INTERLACING PROPERTIES}

Fix a vector $l:=\left(l_{1} ; l_{2}\right)$ where $0 \leq l_{1} \leq m_{1}$ and $0 \leq l_{2} \leq m_{2}$. We define the multi-index $\mathbf{n}^{l}:=\left(\mathbf{n}_{1}+\mathbf{e}^{l_{1}} ; \mathbf{n}_{2}+\mathbf{e}^{l_{2}}\right)=\left(\mathbf{n}_{1}^{l_{1}} ; \mathbf{n}_{2}^{l_{2}}\right)$, where $\mathbf{e}^{l_{i}}$ denotes the unit vector of length $m_{i}+1$ with all components equal to zero except the component $\left(l_{i}+1\right)$ which equals 1 . In this section it is always assumed that both $\mathbf{n}$ and $\mathbf{n}^{l}$ belong to $\mathbb{Z}_{+}^{m_{1}+1}(\bullet) \times \mathbb{Z}_{+}^{m_{2}+1}(\bullet)$.

Fix real constants $A, B$ such that $|A|+|B|>0$ and define

$$
\mathcal{G}_{\mathbf{n}, j}:=A \mathcal{A}_{\mathbf{n}, j}+B \mathcal{A}_{\mathbf{n}^{l}, j}, \quad j=0, \ldots, m_{1} .
$$

Since $\operatorname{deg} a_{\mathbf{n}^{l}, l_{1}}=\operatorname{deg} a_{\mathbf{n}, l_{1}}+1$ it is obvious that $\mathcal{G}_{\mathbf{n}, j} \not \equiv 0, j \leq l_{1}$. In particular, this is always true for $\mathcal{G}_{\mathbf{n}, 0}$.

Lemma 3.1. Assume that $A, B \in \mathbb{R},|A|+|B|>0$, and $n_{1, m_{1}} \geq 1$. Then for all $j \in\left\{0, \ldots, m_{1}\right\}$ such that $n_{1, j} \geq 2, \operatorname{deg} A a_{\mathbf{n}, j}+B a_{\mathbf{n}^{l}, j} \geq n_{1, j}-2$ and $\mathcal{G}_{\mathbf{n}, j} \not \equiv 0$.

Proof. Assume that there exists $j \in\left\{0, \ldots, m_{1}\right\}$ such that $n_{1, j} \geq 2$ and $\operatorname{deg} A a_{\mathbf{n}, j}+B a_{\mathbf{n}^{l}, j} \leq n_{1, j}-3$ $\left(n_{1, j}-3=-1\right.$ means that $\left.A a_{\mathbf{n}, j}+B a_{\mathbf{n}^{l}, j} \equiv 0\right)$. Then $\mathbf{n}_{1}^{l_{1}}-2 \mathbf{e}^{j} \in \mathbb{Z}_{+}^{m_{1}+1}(*)$, where $\mathbf{e}^{j}$ denotes the $m_{1}+1$ dimensional unit vector with all components equal to zero except the component $j+1$ which equals 1. According to Lemma 2.2 the linear form $\mathcal{G}_{\mathbf{n}, 0}$ has at most $\left|\mathbf{n}_{1}\right|-2$ zeros on $\Delta_{0}^{1}$, but $\mathcal{G}_{\mathbf{n}, 0}$ satisfies the same orthogonality relations (2) as $\mathcal{A}_{\mathbf{n}, 0}$ and, therefore, it has at least $\left|\mathbf{n}_{1}\right|-1$ sign changes on this interval. This contradiction implies the statement.

From this lemma it follows that if $n_{1, m_{1}} \geq 2$ then $\mathcal{G}_{\mathbf{n}, j} \not \equiv 0, j \in\left\{0, \ldots, m_{1}\right\}$. 
Lemma 3.2. Assume that $A, B \in \mathbb{R}$ and $\mathcal{G}_{\mathbf{n}, j}=A \mathcal{A}_{\mathbf{n}, j}+B \mathcal{A}_{\mathbf{n}^{l}, j} \not \equiv 0$, for some $j \in\left\{0, \ldots, m_{1}\right\}$. If $j \leq l_{1}$ then $\mathcal{G}_{\mathbf{n}, j}$ has at most $N_{1, j}$ zeros, counting multiplicities, on any interval disjoint from $\Delta_{j+1}^{1}\left(\Delta_{m_{1}+1}^{1}=\emptyset\right)$. If $j>l_{1}$ then $\mathcal{G}_{\mathbf{n}, j}$ has at most $N_{1, j}-1$ zeros, counting multiplicities, on any interval disjoint from $\Delta_{j+1}^{1}$.

Proof. We have

$$
\mathcal{G}_{\mathbf{n}, j}(z)=\sum_{k=j}^{m_{1}}\left(A a_{\mathbf{n}, k}(z)+B a_{\mathbf{n}^{l}, k}(z)\right) \widehat{s}_{j+1, k}^{1}(z),
$$

where $\operatorname{deg} a_{\mathbf{n}, k}=n_{1, k}-1$ and $\operatorname{deg} a_{\mathbf{n}^{l}, k}=n_{1, k}^{l_{1}}-1$. By Lemma $2.2,\left(1, \widehat{s}_{j+1, j+1}^{1}, \ldots, \widehat{s}_{j+1, m_{1}}^{1}\right)$ forms an AT-system with respect to $\left(n_{1, j}^{l_{1}}, \ldots, n_{1, m_{1}}^{l_{1}}\right)$ on any interval disjoint from $\Delta_{j+1}^{1}$, and the result follows immediately.

Notice that for each $j \in\left\{0, \ldots, m_{1}\right\}, \mathcal{G}_{\mathbf{n}, j}$ is a real function when it is restricted to the real line.

Proposition 3.3. Let $n_{1, m_{1}} \geq 1$. Assume that $A, B \in \mathbb{R},|A|+|B|>0$, and let $k=\max \left\{k^{\prime}\right.$ : $\left.\mathcal{G}_{\mathbf{n}, k^{\prime}} \not \equiv 0\right\} \leq m_{1}$. Then, $k \geq l_{1}$ and $\mathcal{G}_{\mathbf{n}, j} \equiv 0, k<j \leq m_{1}$. If $j \leq l_{1}$ then $\mathcal{G}_{\mathbf{n}, j}$ has at most $N_{1, j}$ zeros in $\mathbb{C} \backslash \Delta_{j+1}^{1}$, counting multiplicities, and at least $N_{1, j}-1$ sign changes in the interior of $\Delta_{j}^{1}$. If $l_{1}<j \leq k$ then $\mathcal{G}_{\mathbf{n}, j}$ has at most $N_{1, j}-1$ zeros in $\mathbb{C} \backslash \Delta_{j+1}^{1}$ and at least $N_{1, j}-2$ sign changes in the interior of $\Delta_{j}^{1}$. Therefore, all the zeros of $\mathcal{G}_{\mathbf{n}, j}$ in $\mathbb{C} \backslash \Delta_{j+1}^{1}$ are real and simple.

Proof. If $j \leq l_{1}$, then $\operatorname{deg} a_{\mathbf{n}^{l}, l_{1}}>\operatorname{deg} a_{\mathbf{n}, l_{1}}$ and $\mathcal{G}_{\mathbf{n}, j} \not \equiv 0$. Consequently, $k \geq l_{1}$. Obviously, from the definition of $k, \mathcal{G}_{\mathbf{n}, j} \equiv 0, k<j \leq m_{1}$.

Assume that $\mathcal{G}_{\mathbf{n}, j}, j \leq l_{1}$, has at least $N_{1, j}+1$ zeros in $\mathbb{C} \backslash \Delta_{j+1}^{1}$, counting multiplicities. Select $N_{1, j}+1$ or $N_{1, j}+2$ zeros of $\mathcal{G}_{\mathbf{n}, j}$ which are symmetric with respect to the real axis, and let $Q_{\mathbf{n}, j}^{*}$ be the monic polynomial whose zeros are those prescribed. If $j<l_{1}$ then

$$
\frac{z^{\nu} \mathcal{G}_{\mathbf{n}, j}}{Q_{\mathbf{n}, j}^{*}}=\mathcal{O}\left(\frac{1}{z^{2}}\right), \quad \nu=0, \ldots, N_{1, j+1}
$$

From (9), it follows that

$$
0=\int x^{\nu} \mathcal{G}_{\mathbf{n}, j+1}(x) \frac{d \sigma_{j+1}^{1}(x)}{Q_{\mathbf{n}, j}^{*}(x)}, \quad \nu=0, \ldots, N_{1, j+1} .
$$

These orthogonality relations imply that $\mathcal{G}_{\mathbf{n}, j+1}$ has at least $N_{1, j+1}+1$ zeros on $\Delta_{j+1}^{1}$. Since $\mathcal{G}_{\mathbf{n}, j+1} \not \equiv 0$ we obtain a contradiction with Lemma 3.2 .

If $j=l_{1}$ and $j<k$, then

$$
\frac{z^{\nu} \mathcal{G}_{\mathbf{n}, l_{1}}}{Q_{\mathbf{n}, l_{1}}^{*}}=\mathcal{O}\left(\frac{1}{z^{2}}\right), \quad \nu=0, \ldots, N_{1, l_{1}+1}-1 .
$$

Arguing as before, it follows that $\mathcal{G}_{\mathbf{n}, l_{1}+1}$ has at least $N_{1, l_{1}+1}$ zeros on $\Delta_{l_{1}+1}^{1}$, contradicting Lemma 3.2. If $j=l_{1}=k$ then $\mathcal{G}_{\mathbf{n}, l_{1}+1} \equiv 0$ and $\mathcal{G}_{\mathbf{n}, l_{1}}=A a_{\mathbf{n}, l_{1}}+B a_{\mathbf{n}^{l}, l_{1}}$ is a polynomial of degree at most $n_{1, l_{1}}<N_{1, l_{1}}+1$ and thus it is identically equal to zero which is impossible. Consequently, when $j \leq l_{1}, \mathcal{G}_{\mathbf{n}, j}$ has at most $N_{1, j}$ zeros in $\mathbb{C} \backslash \Delta_{j+1}^{1}$ counting multiplicities. 
Let $l_{1}<j \leq k$ and assume that $\mathcal{G}_{\mathbf{n}, j}$ has at least $N_{1, j}$ zeros in $\mathbb{C} \backslash \Delta_{j+1}^{1}$, counting multiplicities. If $j=m_{1}$ we get immediately a contradiction because in this case $\mathcal{G}_{\mathbf{n}, m_{1}}$ is a polynomial of degree at most $N_{1, m_{1}}-1$. If $l_{1}<j<m_{1}$, then there exists a polynomial $Q_{\mathbf{n}, j}^{*}$ with real coefficients and degree at least $N_{1, j}$ such that

$$
\frac{z^{\nu} \mathcal{G}_{\mathbf{n}, j}}{Q_{\mathbf{n}, j}^{*}}=\mathcal{O}\left(\frac{1}{z^{2}}\right), \quad \nu=0, \ldots, N_{1, j+1}-1
$$

This implies that $\mathcal{G}_{\mathbf{n}, j+1}$ has at least $N_{1, j+1}$ zeros on $\Delta_{j+1}^{1}$ contradicting Lemma 3.2.

Now, let us analyze the sign changes. Notice that $\mathcal{G}_{\mathbf{n}, 0} \not \equiv 0$. Assume that $\mathcal{G}_{\mathbf{n}, 0}$ has $N<N_{1,0}-1=$ $\left|\mathbf{n}_{1}\right|-1$ sign changes on $\Delta_{0}^{1}=\Delta_{0}^{2}$, choose a nonzero linear form

$$
\mathcal{B}_{\mathbf{n}_{2}}(z)=\sum_{k=0}^{m_{2}} b_{\mathbf{n}_{2}, k}(z) \widehat{s}_{1, k}^{2}(z), \quad \operatorname{deg} b_{\mathbf{n}_{2}, k} \leq n_{2, k}-1, \quad k=0, \ldots, m_{2},
$$

such that $\mathcal{B}_{\mathbf{n}_{2}}$ has a zero at each point where $\mathcal{G}_{\mathbf{n}, 0}$ has a sign change, and a zero of order $\left|\mathbf{n}_{2}\right|-1-N$ at one of the extreme points of $\Delta_{0}^{2}$. By Lemma 2.2, $\mathcal{B}_{\mathbf{n}_{2}}$ has at most $\left|\mathbf{n}_{2}\right|-1$ zeros on $\Delta_{0}^{2}$. Thus, $\mathcal{B}_{\mathbf{n}_{2}}$ has exactly those zeros prescribed. By definition,

$$
\int \mathcal{B}_{\mathbf{n}_{2}}(x) \mathcal{G}_{\mathbf{n}, 0}(x) d \sigma_{0}^{2}(x)=0,
$$

which contradicts the fact that $\mathcal{B}_{\mathbf{n}_{2}}(x) \mathcal{G}_{\mathbf{n}, 0}(x)$ has constant sign on $\Delta_{0}^{2}$.

Let us prove by induction that for all $j \leq l_{1}, \mathcal{G}_{\mathbf{n}, j}$ has at least $N_{1, j}-1$ sign changes in the interior of $\Delta_{j}^{1}$. For $j=0$ this was proved above and if $l_{1}=0$ we are done. Let us assume that for some $j<l_{1}, \mathcal{G}_{\mathbf{n}, j}$ has at least $N_{1, j}-1$ sign changes on $\Delta_{j}^{1}$, and let us show that $\mathcal{G}_{\mathbf{n}, j+1}$ has at least $N_{1, j+1}-1$ sign changes on $\Delta_{j+1}^{1}$.

Let $Q_{\mathbf{n}, j}^{*}$ be a monic polynomial whose zeros are $N_{1, j}-1$ points where $\mathcal{G}_{\mathbf{n}, j}$ has a sign change. Then

$$
\frac{z^{\nu} \mathcal{G}_{\mathbf{n}, j}}{Q_{\mathbf{n}, j}^{*}}=\mathcal{O}\left(\frac{1}{z^{2}}\right), \quad \nu=0, \ldots, N_{1, j+1}-2
$$

Using (9), this implies that

$$
0=\int x^{\nu} \mathcal{G}_{\mathbf{n}, j+1}(x) \frac{d \sigma_{j+1}^{1}(x)}{Q_{\mathbf{n}, j}^{*}(x)}, \quad \nu=0, \ldots, N_{1, j+1}-2 .
$$

Thus, $\mathcal{G}_{\mathbf{n}, j+1}$ has at least $N_{1, j+1}-1$ sign changes in the interior of $\Delta_{j+1}^{1}$ as claimed.

Finally, we prove that $\mathcal{G}_{\mathbf{n}, j}, l_{1}<j \leq k$, has at least $N_{1, j}-2$ sign changes in the interior of $\Delta_{j}^{1}$. Let $Q_{\mathbf{n}, l_{1}}^{*}$ be a monic polynomial of degree $N_{1, l_{1}}-1$ whose zeros are points where $\mathcal{G}_{\mathbf{n}, l_{1}}$ changes sign in the interior of $\Delta_{l_{1}}^{1}$, then

$$
\frac{z^{\nu} \mathcal{G}_{\mathbf{n}, l_{1}}}{Q_{\mathbf{n}, l_{1}}^{*}}=\mathcal{O}\left(\frac{1}{z^{2}}\right), \quad \nu=0, \ldots, N_{1, l_{1}+1}-3 .
$$

From here we get orthogonality conditions that imply that $\mathcal{G}_{\mathbf{n}, l_{1}+1}$ has at least $N_{1, l_{1}+1}-2$ sign changes in the interior of $\Delta_{l_{1}+1}^{1}$. One proceeds the same way until we arrive to $j=k$.

From the upper bound on the number of zeros and the lower bound on the number of sign changes it follows that all the zeros are simple and lie on the real line. 
Let $j \in\left\{0, \ldots, m_{2}+1\right\}$. Given two real constants $A, B$, we define

$$
\mathcal{G}_{\mathbf{n},-j}:=A \mathcal{A}_{\mathbf{n},-j}+B \mathcal{A}_{\mathbf{n}^{l},-j} .
$$

Thus, by (13),

$$
\mathcal{G}_{\mathbf{n},-j-1}(z)=\int \frac{\mathcal{G}_{\mathbf{n},-j}(x)}{z-x} d \sigma_{j}^{2}(x), \quad j=0, \ldots, m_{2} .
$$

If $|A|+|B|>0$ then $\mathcal{G}_{\mathbf{n}, 0} \not \equiv 0$ and from (17) it follows that $\mathcal{G}_{\mathbf{n},-j} \not \equiv 0$ for all $j \in\left\{1, \ldots, m_{2}+1\right\}$.

Proposition 3.4. Let $A, B \in \mathbb{R},|A|+|B|>0$. For every $j \in\left\{1, \ldots, m_{2}\right\}$, $\mathcal{G}_{\mathbf{n},-j}$ has at most $N_{2, j}+1$ zeros on $\mathbb{C} \backslash \Delta_{j-1}^{2}$, counting multiplicities, and at least $N_{2, j}$ sign changes in the interior of $\Delta_{j}^{2}$. Hence, all the zeros of $\mathcal{G}_{\mathbf{n},-j}$ on $\mathbb{C} \backslash \Delta_{j-1}^{2}$ are real and simple.

Proof. Let $j \in\left\{0, \ldots, m_{2}\right\}$. By (14) we know that

$$
\int x^{\nu} \mathcal{A}_{\mathbf{n}^{l},-j}(x) d s_{j, k}^{2}(x)=0, \quad k=j, \ldots, m_{2}, \quad \nu=0, \ldots, n_{2, k}^{l_{2}}-1 .
$$

Since $n_{2, k} \leq n_{2, k}^{l_{2}}$, it follows that

$$
\int x^{\nu} \mathcal{G}_{\mathbf{n},-j}(x) d s_{j, k}^{2}(x)=0, \quad k=j, \ldots, m_{2}, \quad \nu=0, \ldots, n_{2, k}-1 .
$$

Using the same arguments employed in the previous section to show that $\mathcal{A}_{\mathbf{n},-j}$ has at least $N_{2, j}$ sign changes in the interior of $\Delta_{j}^{2}$, one obtains the same conclusion for $\mathcal{G}_{\mathbf{n},-j}$.

If $q$ is a polynomial with $\operatorname{deg} q \leq n_{2, j}$, then from (18) we have

$$
\int \frac{q(z)-q(x)}{z-x} \mathcal{G}_{\mathbf{n},-j}(x) d \sigma_{j}^{2}(x)=0 .
$$

Hence, for every $j \in\left\{0, \ldots, m_{2}\right\}$,

$$
\mathcal{G}_{\mathbf{n},-j-1}(z)=\frac{1}{q(z)} \int \frac{q(x)}{z-x} \mathcal{G}_{\mathbf{n},-j}(x) d \sigma_{j}^{2}(x)=\mathcal{O}\left(\frac{1}{z^{n_{2, j}+1}}\right), \quad z \rightarrow \infty .
$$

Assume that for some $j \in\left\{0, \ldots, m_{2}-1\right\}, \mathcal{G}_{\mathbf{n},-j-1}$ has at least $N_{2, j+1}+2$ zeros, counting multiplicities, on $\mathbb{C} \backslash \Delta_{j}^{2}$. Select at least $N_{2, j+1}+2$ zeros of $\mathcal{G}_{\mathbf{n},-j-1}$, symmetric with respect to the real axis, and denote by $Q_{\mathbf{n},-j-1}^{*}$ the monic polynomial whose zeros are the points selected. Then,

$$
\frac{z^{\nu} \mathcal{G}_{\mathbf{n},-j-1}}{Q_{\mathbf{n},-j-1}^{*}}=\mathcal{O}\left(\frac{1}{z^{2}}\right), \quad \nu=0, \ldots, N_{2, j}+1 .
$$

As before, this implies that $\mathcal{G}_{\mathbf{n},-j}$ has at least $N_{2, j}+2$ zeros in the interior of $\Delta_{j}^{2}$. Going downwards on the index $j$, we obtain that $\mathcal{G}_{\mathbf{n}, 0}$ has at least $N_{2,0}+2=N_{1,0}+1$ zeros, which is impossible by Proposition 3.3. Therefore, for all $j \in\left\{1, \ldots, m_{2}+1\right\}, \mathcal{G}_{\mathbf{n}, j}$ has at most $N_{2, j}+1$ zeros in $\mathbb{C} \backslash \Delta_{j-1}^{2}$ and, therefore, they must be real and simple.

Theorem 3.5. Let $\mathbf{n}, \mathbf{n}^{l} \in \mathbb{Z}_{+}^{m_{1}+1}(\bullet) \times \mathbb{Z}_{+}^{m_{2}+1}(\bullet), n_{1, m_{1}} \geq 2$. Then, for all $j \in\left\{-m_{2}, \ldots, m_{1}\right\}$ the zeros of $\mathcal{A}_{\mathbf{n}, j}$ and $\mathcal{A}_{\mathbf{n}^{l}, j}$ interlace; that is, between two consecutive zeros of $\mathcal{A}_{\mathbf{n}, j}$ there is one zero of $\mathcal{A}_{\mathbf{n}^{l}, j}$ and viceversa. 
Proof. Since $n_{1, m_{1}} \geq 2$, from Lemma 3.1 we know that for all $j \in\left\{0, \ldots, m_{1}\right\}$ and for all $A, B$ real such that $|A|+|B|>0$, the linear form $\mathcal{G}_{\mathbf{n}, j}$ is not identically equal to zero. This is always true for $j \in\left\{-m_{2}, \ldots,-1\right\}$. Therefore, from Propositions 3.3 and 3.4 we know that for all real $A, B$, such that $|A|+|B|>0$ the zeros of $\mathcal{G}_{\mathbf{n}, j}, j \in\left\{-m_{2}, \ldots, m_{1}\right\}$, are real and simple. This is the basic fact we will use in the proof.

Fix $y \in \mathbb{R} \backslash \Delta_{j+1}^{1}$. It cannot occur that $\mathcal{A}_{\mathbf{n}, j}(y)=\mathcal{A}_{\mathbf{n}^{l}, j}(y)=0$. If so, $y$ would be a simple zero of $\mathcal{A}_{\mathbf{n}, j}$ and $\mathcal{A}_{\mathbf{n}^{l}, j}$. Thus, $\mathcal{A}_{\mathbf{n}, j}^{\prime}(y) \neq 0$ and $\mathcal{A}_{\mathbf{n}^{l}, j}^{\prime}(y) \neq 0$. Take $A=1$ and $B=-\mathcal{A}_{\mathbf{n}, j}^{\prime}(y) / \mathcal{A}_{\mathbf{n}^{l}, j}^{\prime}(y)$ and consider $\mathcal{G}_{\mathbf{n}, j}=A \mathcal{A}_{\mathbf{n}, j}+B \mathcal{A}_{\mathbf{n}^{l}, j}$. With this choice of $A$ and $B$, we have

$$
\mathcal{G}_{\mathbf{n}, j}(y)=\mathcal{G}_{\mathbf{n}, j}^{\prime}(y)=0,
$$

and we obtain a contradiction because the zeros of $\mathcal{G}_{\mathbf{n}, j}$ are simple.

Now, taking $A=\mathcal{A}_{\mathbf{n}^{l}, j}(y)$ and $B=-\mathcal{A}_{\mathbf{n}, j}(y)$, we have that $|A|+|B|>0$. Since

$$
\mathcal{A}_{\mathbf{n}^{l}, j}(y) \mathcal{A}_{\mathbf{n}, j}(y)-\mathcal{A}_{\mathbf{n}, j}(y) \mathcal{A}_{\mathbf{n}^{l}, j}(y)=0,
$$

and the zeros on $\mathbb{R} \backslash \Delta_{j+1}^{1}$ of $\mathcal{A}_{\mathbf{n}^{l}, j}(y) \mathcal{A}_{\mathbf{n}, j}(x)-\mathcal{A}_{\mathbf{n}, j}(y) \mathcal{A}_{\mathbf{n}^{l}, j}(x)$ with respect to $x$ are simple, it follows that

$$
\mathcal{A}_{\mathbf{n}^{l}, j}(y) \mathcal{A}_{\mathbf{n}, j}^{\prime}(y)-\mathcal{A}_{\mathbf{n}, j}(y) \mathcal{A}_{\mathbf{n}^{l}, j}^{\prime}(y) \neq 0 .
$$

But $\mathcal{A}_{\mathbf{n}^{l}, j}(y) \mathcal{A}_{\mathbf{n}, j}^{\prime}(y)-\mathcal{A}_{\mathbf{n}, j}(y) \mathcal{A}_{\mathbf{n}^{l}, j}^{\prime}(y)$ is a continuous real function on $\mathbb{R} \backslash \Delta_{j+1}^{1}$ in $y$ so it must have constant sign on each one of the connected components of $\mathbb{R} \backslash \Delta_{j+1}^{1}$. In particular, its sign on $\Delta_{j}^{1}$ is constant.

Evaluating $\mathcal{A}_{\mathbf{n}^{l}, j}(y) \mathcal{A}_{\mathbf{n}, j}^{\prime}(y)-\mathcal{A}_{\mathbf{n}, j}(y) \mathcal{A}_{\mathbf{n}^{l}, j}^{\prime}(y)$ at two consecutive zeros of $\mathcal{A}_{\mathbf{n}^{l}, j}$, since the sign of $\mathcal{A}_{\mathbf{n}^{l}, j}^{\prime}$ at these two points changes, the sign of $\mathcal{A}_{\mathbf{n}, j}$ must also change. Using Bolzano's theorem we find that there must be an intermediate zero of $\mathcal{A}_{\mathbf{n}, j}$. Analogously, one proves that between two consecutive zeros of $\mathcal{A}_{\mathbf{n}, j}$ on $\Delta_{j}^{1}$ there is one of $\mathcal{A}_{\mathbf{n}^{l}, j}$. Thus, the interlacing property has been proved.

\section{Asymptotic Distribution of Zeros}

Let $\left\{\mu_{l}\right\} \subset \mathcal{M}(E)$ be a sequence of positive measures, where $E$ is a compact subset of the complex plane and $\mu \in \mathcal{M}(E)$. We write

$$
* \lim _{l} \mu_{l}=\mu,
$$

if for every continuous function $f \in \mathcal{C}(E)$

$$
\lim _{l} \int f d \mu_{l}=\int f d \mu
$$

that is, when the sequence of measures converges to $\mu$ in the weak star topology. Given a polynomial $q_{l}$ of degree $l \geq 1$, we denote the associated normalized zero counting measure by

$$
\mu_{q_{l}}=\frac{1}{l} \sum_{q_{l}(x)=0} \delta_{x}
$$


where $\delta_{x}$ is the Dirac measure with mass 1 at $x$ (in the sum the zeros are repeated according to their multiplicity).

Lemma 4.1. Let $E \subset \mathbb{C}$ be a compact set which is regular with respect to the Dirichlet problem and $\phi$ a continuous function on $E$. Then there exists a unique $\bar{\mu} \in \mathcal{M}_{1}(E)$ and a constant $w$ such that

$$
V^{\bar{\mu}}(z)+\phi(z) \begin{cases}\leq w, & z \in \operatorname{supp}(\bar{\mu}) \\ \geq w, & z \in E\end{cases}
$$

If the compact set $E$ is not regular with respect to the Dirichlet problem then the second part of the statement is true except on a set $e$ such that $\operatorname{cap}(e)=0$. Theorem I.1.3 in [27] contains a proof of this lemma in this context. When $E$ is regular, it is well known that this inequality except for a set of capacity zero implies the inequality for all points in the set (cf. Theorem I.4.8 from [27]). $\bar{\mu}$ is called the equilibrium measure in the presence of the external field $\phi$ on $E$ and $w$ is the equilibrium constant.

In order to determine the asymptotic zero distribution of the polynomials $Q_{\mathbf{n}, j}$ we use the following lemma. Different versions of it appear in [5], [10], and [28]. In [10], it was proved assuming that $\operatorname{supp}(\sigma)$ is an interval on which $\sigma^{\prime}>0$ a.e. We wish to preserve this more restrictive condition for stronger results in section 6. Theorem 3.3.3 in [28] and Theorem 1 in [5], do not cover the type of external field we consider here. So, we will sketch a proof.

Lemma 4.2. Let $\sigma \in \mathbf{R e g}, \operatorname{supp}(\sigma) \subset \mathbb{R}$, where $\operatorname{supp}(\sigma)$ is regular with respect to the Dirichlet problem. Let $\left\{\phi_{l}\right\}, l \in \Lambda \subset \mathbb{Z}_{+}$, be a sequence of positive continuous functions on $\operatorname{supp}(\sigma)$ such that

$$
\lim _{l \in \Lambda} \frac{1}{2 l} \log \frac{1}{\left|\phi_{l}(x)\right|}=\phi(x)>-\infty,
$$

uniformly on $\operatorname{supp}(\sigma) . B y\left\{q_{l}\right\}, l \in \Lambda$, denote a sequence of monic polynomials such that $\operatorname{deg} q_{l}=l$ and

$$
\int x^{k} q_{l}(x) \phi_{l}(x) d \sigma(x)=0, \quad k=0, \ldots, l-1 .
$$

Then

$$
* \lim _{l \in \Lambda} \mu_{q_{l}}=\bar{\mu},
$$

and

$$
\lim _{l \in \Lambda}\left(\int\left|q_{l}(x)\right|^{2} \phi_{l}(x) d \sigma(x)\right)^{1 / 2 l}=e^{-w}
$$

where $\bar{\mu}$ and $w$ are the equilibrium measure and equilibrium constant in the presence of the external field $\phi$ on $\operatorname{supp}(\sigma)$. We also have that

$$
\lim _{l \in \Lambda}\left(\frac{\left|q_{l}(z)\right|}{\left\|q_{l} \phi_{l}^{1 / 2}\right\|_{E}}\right)^{1 / l}=\exp \left(w-V^{\bar{\mu}}(z)\right), \quad \mathcal{K} \subset \mathbb{C} \backslash \operatorname{Co}(\operatorname{supp}(\sigma)) .
$$


Proof. On account of (19) and Lemma 4.1, it follows that for any $\varepsilon>0$ there exists $l_{0}$ such that for all $l \geq l_{0}, l \in \Lambda$, and $z \in \operatorname{supp}(\bar{\mu}) \subset \operatorname{supp}(\sigma)=: E$

$$
\frac{1}{l} \log \frac{\left|p_{l}(z)\right|}{\left\|p_{l} \phi_{l}^{1 / 2}\right\|_{E}} \leq \frac{1}{2 l} \log \frac{1}{\left|\phi_{l}(z)\right|} \leq \phi(z)+\varepsilon \leq w-V^{\bar{\mu}}(z)+\varepsilon,
$$

where $\left\{p_{l}\right\}, l \in \Lambda$, is any sequence of monic polynomials such that $\operatorname{deg} p_{l}=l$ and $\left\|p_{l} \phi_{l}^{1 / 2}\right\|_{E}=$ $\max _{z \in E}\left|\left(p_{l} \phi_{l}^{1 / 2}\right)(z)\right|$. Hence,

$$
u_{l}(z):=V^{\bar{\mu}}(z)+\frac{1}{l} \log \frac{\left|p_{l}(z)\right|}{\left\|p_{l} \phi_{l}^{1 / 2}\right\|_{E}} \leq w+\varepsilon, \quad z \in \operatorname{supp}(\bar{\mu}), \quad l \geq l_{0} .
$$

Since $u_{l}$ is subharmonic in $\overline{\mathbb{C}} \backslash \operatorname{supp}(\bar{\mu})$, by the continuity and maximum principles, we have

$$
u_{l}(z) \leq w+\varepsilon, \quad z \in \overline{\mathbb{C}}, \quad l \geq l_{0} .
$$

In particular,

$$
u_{l}(\infty)=\frac{1}{l} \log \frac{1}{\left\|p_{l} \phi_{l}^{1 / 2}\right\|_{E}} \leq w+\varepsilon .
$$

The last two relations imply

$$
\limsup _{l \in \Lambda}\left(\frac{\left|p_{l}(z)\right|}{\left\|p_{l} \phi_{l}^{1 / 2}\right\|_{E}}\right)^{1 / l} \leq \exp \left(w-V^{\bar{\mu}}(z)\right), \quad \mathcal{K} \subset \mathbb{C},
$$

and

$$
\liminf _{l \in \Lambda}\left\|p_{l} \phi_{l}^{1 / 2}\right\|_{E}^{1 / l} \geq \exp (-w) .
$$

In particular, these relations hold for the sequence of polynomials $\left\{q_{l}\right\}, l \in \Lambda$.

Let $t_{l}$ be the weighted Fekete polynomial of degree $l$ for the weight $e^{-\phi}$ on $\operatorname{supp}(\sigma)$ and $|\sigma|$ be the total variation of $\sigma$. From the minimality property in the $L_{2}$ norm of $q_{l}$, we have

$$
\begin{gathered}
\left\|q_{l} \phi_{l}^{1 / 2}\right\|_{2}:=\left(\int\left|q_{l}(x)\right|^{2} \phi_{l}(x) d \sigma(x)\right)^{1 / 2} \leq\left\|t_{l} \phi_{l}^{1 / 2}\right\|_{2} \leq|\sigma|^{1 / 2}\left\|t_{l} \phi_{l}^{1 / 2}\right\|_{E} \leq \\
|\sigma|^{1 / 2}\left\|t_{l} e^{-l \phi}\right\|_{E}\left\|\phi_{l}^{1 / 2} e^{l \phi}\right\|_{E} .
\end{gathered}
$$

Then, using (19) and Theorem III.1.9 in [27], we obtain that

$$
\limsup _{l \in \Lambda}\left\|q_{l} \phi_{l}^{1 / 2}\right\|_{2}^{1 / l} \leq e^{-w} .
$$

Since $\operatorname{supp}(\sigma)$ is regular with respect to the Dirichlet problem, Theorem 3.2.3 vi) in [28] yields

$$
\limsup _{l \in \Lambda}\left(\frac{\left\|q_{l} \phi_{l}^{1 / 2}\right\|_{E}}{\left\|q_{l} \phi_{l}^{1 / 2}\right\|_{2}}\right)^{1 / l} \leq 1
$$

which combined with (25) (with $p_{l}=q_{l}$ ) and (26) implies

$$
\lim _{l \in \Lambda}\left(\frac{\left\|q_{l} \phi_{l}^{1 / 2}\right\|_{E}}{\left\|q_{l} \phi_{l}^{1 / 2}\right\|_{2}}\right)^{1 / l}=1 .
$$

Thus, we obtain (22) since (25), (26), and (27) give

$$
\limsup _{l \in \Lambda}\left\|q_{l} \phi_{l}^{1 / 2}\right\|_{E}^{1 / l}=\limsup _{l \in \Lambda}\left\|q_{l} \phi_{l}^{1 / 2}\right\|_{2}^{1 / l}=e^{-w} .
$$


All the zeros of $q_{l}$ lie in $\operatorname{Co}(\operatorname{supp}(\sigma)) \subset \mathbb{R}$. The unit ball in the weak star topology of measures is compact. Take any subsequence of indices $\Lambda^{\prime} \subset \Lambda$ such that

$$
* \lim _{l \in \Lambda^{\prime}} \mu_{q_{l}}=\mu_{\Lambda^{\prime}}
$$

Then,

$$
\lim _{l \in \Lambda^{\prime}} \frac{1}{l} \log \left|q_{l}(z)\right|=-\lim _{n \in \Lambda^{\prime}} \int \log \frac{1}{|z-x|} \mu_{q_{l}}(x)=-V^{\mu_{\Lambda^{\prime}}(z), \quad \mathcal{K} \subset \mathbb{C} \backslash \operatorname{Co}(\operatorname{supp}(\sigma)) .}
$$

This, together with (22) and (24) (applied to $\left\{q_{l}\right\}, l \in \Lambda^{\prime}$ ), implies

$$
\left(V^{\bar{\mu}}-V^{\mu_{\Lambda^{\prime}}}\right)(z) \leq 0, \quad z \in \overline{\mathbb{C}} \backslash \operatorname{Co}(\operatorname{supp}(\sigma))
$$

Since $V^{\bar{\mu}}-V^{\mu_{\Lambda^{\prime}}}$ is subharmonic in $\overline{\mathbb{C}} \backslash \operatorname{supp}(\bar{\mu})$ and $\left(V^{\bar{\mu}}-V^{\mu_{\Lambda^{\prime}}}\right)(\infty)=0$, from the maximum principle, it follows that $V^{\bar{\mu}} \equiv V^{\mu_{\Lambda^{\prime}}}$ in $\mathbb{C} \backslash \operatorname{Co}(\operatorname{supp}(\sigma))$ and thus $\mu_{\Lambda^{\prime}}=\bar{\mu}$. Consequently, (21) holds. (21) and (22) imply (23).

Using Lemma 4.2, we can obtain the asymptotic limit distribution of the zeros of the polynomials $Q_{\mathbf{n}, j}, j=-m_{2}, \ldots, m_{1}$. At this point, let us make a slight change of notation. In the sequel,

$$
\begin{gathered}
\Delta_{j}=\Delta_{j}^{1}, \quad \sigma_{j}=\sigma_{j}^{1}, \quad j=0,1, \ldots, m_{1}, \\
\Delta_{j}=\Delta_{-j}^{2}, \quad \sigma_{j}=\sigma_{-j}^{2}, \quad j=0,-1, \ldots,-m_{2},
\end{gathered}
$$

and

$$
N_{\mathbf{n}, j}= \begin{cases}N_{1, j}(\mathbf{n})-1, & j=0,1 \ldots, m_{1}, \\ N_{2,-j}(\mathbf{n}), & j=0,-1, \ldots,-m_{2} .\end{cases}
$$

According to Propositions 2.5 and 2.7, for all $j=-m_{2}, \ldots, m_{1}$ the zeros of $Q_{\mathbf{n}, j}$ are all simple, lie in the interior of $\Delta_{j}$, and total $N_{\mathbf{n}, j}$ points.

Theorem 4.3. Let $\Lambda=\Lambda\left(p_{1,0}, \ldots, p_{1, m_{1}} ; p_{2,0}, \ldots, p_{2, m_{2}}\right) \subset \mathbb{Z}_{+}^{m_{1}+1}(\bullet) \times \mathbb{Z}_{+}^{m_{2}+1}(\bullet),\left(S^{1}, S^{2}\right) \in \mathbf{R e g}$, $S^{1}=\mathcal{N}\left(\sigma_{0}^{1}, \ldots, \sigma_{m_{1}}^{1}\right)$, and $S^{2}=\mathcal{N}\left(\sigma_{0}^{2}, \ldots, \sigma_{m_{2}}^{2}\right)$ be given. Then

$$
* \lim _{\mathbf{n} \in \Lambda} \mu_{Q_{\mathbf{n}, j}}=\bar{\mu}_{j}, \quad j=-m_{2}, \ldots, m_{1},
$$

where $\bar{\mu}=\bar{\mu}(\mathcal{C}) \in \mathcal{M}_{1}$ is the vector equilibrium measure determined by the matrix $\mathcal{C}$ in (4) on the system of compact sets $E_{j}=\operatorname{supp}\left(\sigma_{j}^{1}\right), j=0, \ldots, m_{1}, E_{j}=\operatorname{supp}\left(\sigma_{-j}^{2}\right), j=-m_{2}, \ldots, 0$. Moreover,

$$
\lim _{\mathbf{n} \in \Lambda}\left(\int \frac{Q_{\mathbf{n}, j}^{2}(x)}{\left|Q_{\mathbf{n}, j-1}(x)\right|} \frac{\left|\mathcal{A}_{\mathbf{n}, j}(x)\right|}{\left|Q_{\mathbf{n}, j}(x)\right|} d\left|\sigma_{j}\right|(x)\right)^{1 / 2\left|\mathbf{n}_{1}\right|}=\exp \left(-\sum_{k=j}^{m_{1}} \omega_{k}^{\bar{\mu}} / P_{k}\right),
$$

where the $\omega_{k}^{\bar{\mu}}$ denote the corresponding equilibrium constants. 
Proof. The unit ball in the cone of positive Borel measures is weak star compact; therefore, it is sufficient to show that each one of the sequences of measures $\left\{\mu_{Q_{\mathbf{n}, j}}\right\}, \mathbf{n} \in \Lambda, j=-m_{2}, \ldots, m_{1}$, has only one accumulation point which coincides with the corresponding component of the vector measure $\bar{\mu}(\mathcal{C})$. Let $\Lambda^{\prime} \subset \Lambda$ be a subsequence of multi-indices such that for each $j=-m_{2}, \ldots, m_{1}$

$$
* \lim _{\mathbf{n} \in \Lambda^{\prime}} \mu_{Q_{\mathbf{n}, j}}=\mu_{j}
$$

Notice that $\mu_{j} \in \mathcal{M}_{1}\left(E_{j}\right), j=-m_{2}, \ldots, m_{1}$. Therefore,

$$
\lim _{\mathbf{n} \in \Lambda^{\prime}}\left|Q_{\mathbf{n}, j}(z)\right|^{1 /\left|\mathbf{n}_{1}\right|}=\exp \left(-P_{j} V^{\mu_{j}}(z)\right)
$$

uniformly on compact subsets of $\mathbb{C} \backslash \Delta_{j}$, where $P_{j}=\lim _{\mathbf{n} \in \Lambda^{\prime}} N_{\mathbf{n}, j} /\left|\mathbf{n}_{1}\right|$.

Because of the normalization adopted on $a_{\mathbf{n}, m_{1}}, \mathcal{A}_{\mathbf{n}, m_{1}}=Q_{\mathbf{n}, m_{1}}$; consequently, when $j=m_{1}$, (11) takes the form

$$
\int x^{\nu} Q_{\mathbf{n}, m_{1}}(x) \frac{d\left|\sigma_{m_{1}}\right|(x)}{\left|Q_{\mathbf{n}, m_{1}-1}(x)\right|}=0, \quad \nu=0, \ldots, N_{\mathbf{n}, m_{1}}-1 .
$$

(By $|\sigma|$ we denote the total variation of the measure $\sigma$.) According to (31)

$$
\lim _{\mathbf{n} \in \Lambda^{\prime}} \frac{1}{2 N_{\mathbf{n}, m_{1}}} \log \left|Q_{\mathbf{n}, m_{1}-1}(x)\right|=-\frac{P_{m_{1}-1}}{2 P_{m_{1}}} V^{\mu_{m_{1}-1}}(x),
$$

uniformly on $\Delta_{m_{1}}$. Using Lemma 4.2 , it follows that $\mu_{m_{1}}$ is the unique solution of the extremal problem

$$
V^{\mu_{m_{1}}}(x)-\frac{P_{m_{1}-1}}{2 P_{m_{1}}} V^{\mu_{m_{1}-1}}(x) \begin{cases}=\omega_{m_{1}}, & x \in \operatorname{supp}\left(\mu_{m_{1}}\right) \\ \geq \omega_{m_{1}}, & x \in E_{m_{1}}\end{cases}
$$

and

$$
\lim _{\mathbf{n} \in \Lambda^{\prime}}\left(\int \frac{Q_{\mathbf{n}, m_{1}}^{2}(x)}{\left|Q_{\mathbf{n}, m_{1}-1}(x)\right|} d\left|\sigma_{m_{1}}\right|(x)\right)^{1 / 2 N_{\mathbf{n}, m_{1}}}=e^{-\omega_{m_{1}}}
$$

Let us show by induction on decreasing values of $j$, that for all $j \in\left\{-m_{2}, \ldots, m_{1}\right\}$

$$
V^{\mu_{j}}(x)-\frac{P_{j-1}}{2 P_{j}} V^{\mu_{j-1}}(x)-\frac{P_{j+1}}{2 P_{j}} V^{\mu_{j+1}}(x)+\frac{P_{j+1}}{P_{j}} \omega_{j+1} \begin{cases}=\omega_{j}, & x \in \operatorname{supp}\left(\mu_{j}\right) \\ \geq \omega_{j}, & x \in E_{j}\end{cases}
$$

where $P_{-m_{2}-1}=P_{m_{1}+1}=0$, and

$$
\lim _{\mathbf{n} \in \Lambda^{\prime}}\left(\int \frac{Q_{\mathbf{n}, j}^{2}(x)}{\left|Q_{\mathbf{n}, j-1}(x)\right|} \frac{\left|\mathcal{A}_{\mathbf{n}, j}(x)\right|}{\left|Q_{\mathbf{n}, j}(x)\right|} d\left|\sigma_{j}\right|(x)\right)^{1 / 2 N_{\mathbf{n}, j}}=e^{-\omega_{j}}
$$

where $Q_{\mathbf{n},-m_{2}-1} \equiv 1$. For $j=m_{1}$ these relations are non other than (32)-(33) and the initial induction step is settled. Let us assume that the statement is true for $j+1 \in\left\{-m_{2}+1, \ldots, m_{1}\right\}$ and let us prove it for $j$.

It is easy to see that the orthogonality relations (11) and (15) can be expressed as

$$
\int x^{\nu} Q_{\mathbf{n}, j}(x) \frac{\left|Q_{\mathbf{n}, j+1}(x) \mathcal{A}_{\mathbf{n}, j}(x)\right|}{\left|Q_{\mathbf{n}, j}(x)\right|} \frac{d\left|\sigma_{j}\right|(x)}{\left|Q_{\mathbf{n}, j-1}(x) Q_{\mathbf{n}, j+1}(x)\right|}=0, \quad \nu=0, \ldots, N_{\mathbf{n}, j}-1 .
$$


On account of (12) and (16) taking $q=Q_{\mathbf{n}, j+1}$, this can be further transformed into

$$
\int x^{\nu} Q_{\mathbf{n}, j}(x)\left(\int \frac{Q_{\mathbf{n}, j+1}^{2}(t)}{\left|Q_{\mathbf{n}, j}(t)\right|} \frac{\left|\mathcal{A}_{\mathbf{n}, j+1}(t)\right|}{\left|Q_{\mathbf{n}, j+1}(t)\right|} \frac{d\left|\sigma_{j+1}\right|(t)}{|x-t|}\right) \frac{d\left|\sigma_{j}\right|(x)}{\left|Q_{\mathbf{n}, j-1}(x) Q_{\mathbf{n}, j+1}(x)\right|}=0,
$$

for $\nu=0, \ldots, N_{\mathbf{n}, j}-1$.

Relation (31) implies that

$$
\lim _{\mathbf{n} \in \Lambda^{\prime}} \frac{1}{2 N_{\mathbf{n}, j}} \log \left|Q_{\mathbf{n}, j-1}(x) Q_{\mathbf{n}, j+1}(x)\right|=-\frac{P_{j-1}}{2 P_{j}} V^{\mu_{j-1}}(x)-\frac{P_{j+1}}{2 P_{j}} V^{\mu_{j+1}}(x),
$$

uniformly on $\Delta_{j}$. (Since $Q_{\mathbf{n},-m_{2}-1} \equiv 1$, when $j=-m_{2}$ we only get the second term on the right hand side of this limit; that is, $P_{-m_{2}-1}=0$.)

Set

$$
K_{\mathbf{n}, j+1}=\left(\int \frac{Q_{\mathbf{n}, j+1}^{2}(t)}{\left|Q_{\mathbf{n}, j}(t)\right|} \frac{\left|\mathcal{A}_{\mathbf{n}, j+1}(t)\right|}{\left|Q_{\mathbf{n}, j+1}(t)\right|} d\left|\sigma_{j+1}\right|(t)\right)^{-1 / 2} .
$$

It follows that for all $x \in \Delta_{j}$

$$
\frac{1}{\delta_{j+1}^{*} K_{\mathbf{n}, j+1}^{2}} \leq \int \frac{Q_{\mathbf{n}, j+1}^{2}(t)}{\left|Q_{\mathbf{n}, j}(t)\right|} \frac{\left|\mathcal{A}_{\mathbf{n}, j+1}(t)\right|}{\left|Q_{\mathbf{n}, j+1}(t)\right|} \frac{d\left|\sigma_{j+1}\right|(t)}{|x-t|} \leq \frac{1}{\delta_{j+1} K_{\mathbf{n}, j+1}^{2}},
$$

where $0<\delta_{j+1}=\inf \left\{|x-t|: t \in \Delta_{j+1}, x \in \Delta_{j}\right\} \leq \max \left\{|x-t|: t \in \Delta_{j+1}, x \in \Delta_{j}\right\}=\delta_{j+1}^{*}<\infty$.

Taking into consideration these inequalities, from the induction hypothesis, we obtain that

$$
\lim _{\mathbf{n} \in \Lambda^{\prime}}\left(\int \frac{Q_{\mathbf{n}, j+1}^{2}(t)}{\left|Q_{\mathbf{n}, j}(t)\right|} \frac{\left|\mathcal{A}_{\mathbf{n}, j+1}(t)\right|}{\left|Q_{\mathbf{n}, j+1}(t)\right|} \frac{d\left|\sigma_{j+1}\right|(t)}{|x-t|}\right)^{1 / 2 N_{\mathbf{n}, j}}=e^{-P_{j+1} \omega_{j+1} / P_{j}}
$$

Taking (36) and (37) into account, Lemma 4.2 yields that $\mu_{j}$ is the unique solution of the extremal problem (34) and

$$
\lim _{\mathbf{n} \in \Lambda^{\prime}}\left(\iint \frac{Q_{\mathbf{n}, j+1}^{2}(t)}{\left|Q_{\mathbf{n}, j}(t)\right|} \frac{\left|\mathcal{A}_{\mathbf{n}, j+1}(t)\right|}{\left|Q_{\mathbf{n}, j+1}(t)\right|} \frac{d\left|\sigma_{j+1}\right|(t)}{|x-t|} \frac{Q_{\mathbf{n}, j}^{2}(x) d\left|\sigma_{j}\right|(x)}{\left|Q_{\mathbf{n}, j-1}(x) Q_{\mathbf{n}, j+1}(x)\right|}\right)^{1 / 2 N_{\mathbf{n}, j}}=e^{-\omega_{j}}
$$

According to (12) and (16) with $q=Q_{\mathbf{n}, j+1}$

$$
\frac{1}{\left|Q_{\mathbf{n}, j+1}(x)\right|} \int \frac{Q_{\mathbf{n}, j+1}^{2}(t)}{\left|Q_{\mathbf{n}, j}(t)\right|} \frac{\left|\mathcal{A}_{\mathbf{n}, j+1}(t)\right|}{\left|Q_{\mathbf{n}, j+1}(t)\right|} \frac{d\left|\sigma_{j+1}\right|(t)}{|x-t|}=\frac{\left|\mathcal{A}_{\mathbf{n}, j}(x)\right|}{\left|Q_{\mathbf{n}, j}(x)\right|}, \quad x \in \Delta_{j},
$$

which allows to reduce the previous formula to (35) thus concluding the induction.

Now, we can rewrite (34) multiplying through by $P_{j}^{2}$ and taking the constant term on the left to the right to obtain the system of boundary value equations

$$
P_{j}^{2} V^{\mu_{j}}(x)-\frac{P_{j-1} P_{j}}{2} V^{\mu_{j-1}}(x)-\frac{P_{j} P_{j+1}}{2} V^{\mu_{j+1}}(x) \begin{cases}=\omega_{j}^{\prime}, & x \in \operatorname{supp}\left(\mu_{j}\right), \\ \geq \omega_{j}^{\prime}, & x \in E_{j},\end{cases}
$$

for $j=-m_{2}, \ldots, m_{1}$, where

$$
\omega_{j}^{\prime}=P_{j}^{2} \omega_{j}-P_{j} P_{j+1} \omega_{j+1}
$$

The terms with $P_{-m_{2}-1}$ and $P_{m_{1}+1}$ do not appear when $j=-m_{2}$ and $j=m_{1}$, respectively. By Lemma $1.2,\left(\mu_{-m_{2}}, \ldots, \mu_{m_{1}}\right)=\left(\bar{\mu}_{-m_{2}}, \ldots, \bar{\mu}_{m_{1}}\right)$ and $\left(\omega_{-m_{2}}^{\prime}, \ldots, \omega_{m_{1}}^{\prime}\right)=\left(\omega_{-m_{2}}^{\bar{\mu}}, \ldots, \omega_{m_{1}}^{\bar{\mu}}\right)$ for any convergent subsequence showing the existence of the limits in (29) as stated. 
Notice that (35) implies that

$$
\lim _{\mathbf{n} \in \Lambda^{\prime}}\left(\int \frac{Q_{\mathbf{n}, j}^{2}(x)}{\left|Q_{\mathbf{n}, j-1}(x)\right|} \frac{\left|\mathcal{A}_{\mathbf{n}, j}(x)\right|}{\left|Q_{\mathbf{n}, j}(x)\right|} d\left|\sigma_{j}\right|(x)\right)^{1 / 2\left|\mathbf{n}_{1}\right|}=e^{-P_{j} \omega_{j}}
$$

On the other hand, from (39) it follows that $P_{m_{1}} \omega_{m_{1}}=\omega_{m_{1}}^{\bar{\mu}} / P_{m_{1}}$ when $j=m_{1}$. Suppose that $P_{j+1} \omega_{j+1}=\sum_{k=j+1}^{m_{1}} \frac{\omega_{k}^{\bar{\mu}}}{P_{k}}, j+1 \in\left\{-m_{2}+1, \ldots, m_{1}\right\}$. Then, according to (39)

$$
P_{j} \omega_{j}=\frac{\omega_{j}^{\bar{\mu}}}{P_{j}}+P_{j+1} \omega_{j+1}=\sum_{k=j}^{m_{1}} \frac{\omega_{k}^{\bar{\mu}}}{P_{k}}
$$

and (30) immediately follows.

\section{Proof of Theorem 1}

Here, we maintain the change of notation introduced in the previous section. Theorem 1.3 is a consequence of the following more general result.

Theorem 5.1. Let $\Lambda=\Lambda\left(p_{1,0}, \ldots, p_{1, m_{1}} ; p_{2,0}, \ldots, p_{2, m_{2}}\right) \subset \mathbb{Z}_{+}^{m_{1}+1}(\bullet) \times \mathbb{Z}_{+}^{m_{2}+1}(\bullet),\left(S^{1}, S^{2}\right) \in \mathbf{R e g}$, $S^{1}=\mathcal{N}\left(\sigma_{0}^{1}, \ldots, \sigma_{m_{1}}^{1}\right)$, and $S^{2}=\mathcal{N}\left(\sigma_{0}^{2}, \ldots, \sigma_{m_{2}}^{2}\right)$ be given. Let $\left\{\mathcal{A}_{\mathbf{n}, j}\right\}, \mathbf{n} \in \Lambda, j=-m_{2}-1, \ldots, m_{1}$, be the sequences of "monic" linear forms associated with the corresponding mixed type orthogonal polynomials. Then, for each $j=-m_{2}-1, \ldots, m_{1}$

$$
\lim _{\mathbf{n} \in \Lambda}\left|\mathcal{A}_{\mathbf{n}, j}(z)\right|^{1 /\left|\mathbf{n}_{1}\right|}=G_{j}(z), \quad \mathcal{K} \subset \mathbb{C} \backslash\left(\Delta_{j} \cup \Delta_{j+1}\right)
$$

$\left(\Delta_{-m_{2}-1}=\Delta_{m_{1}+1}=\emptyset\right)$, where

$$
G_{j}(z)=\exp \left(P_{j+1} V^{\bar{\mu}_{j+1}}(z)-P_{j} V^{\bar{\mu}_{j}}(z)-2 \sum_{k=j+1}^{m_{1}} \frac{\omega_{k}^{\bar{\mu}}}{P_{k}}\right), \quad j=-m_{2}-1, \ldots, m_{1}-1,
$$

$\left(P_{-m_{2}-1}=P_{m_{1}+1}=0\right)$ and

$$
G_{m_{1}}(z)=\exp \left(-P_{m_{1}} V^{\bar{\mu}_{m_{1}}}(z)\right)
$$

$\bar{\mu}=\bar{\mu}(\mathcal{C})=\left(\bar{\mu}_{-m_{2}}, \ldots, \bar{\mu}_{m_{1}}\right)$ is the equilibrium vector measure and $\left(\omega_{-m_{2}}^{\bar{\mu}}, \ldots, \omega_{m_{1}}^{\bar{\mu}}\right)$ is the system of equilibrium constants for the vector potential problem determined by the interaction matrix $\mathcal{C}$ defined in (4) on the system of compact sets $E_{j}=\operatorname{supp}\left(\sigma_{j}^{1}\right), j=0, \ldots, m_{1}, E_{j}=\operatorname{supp}\left(\sigma_{-j}^{2}\right), j=$ $-m_{2}, \ldots, 0$.

Proof. If $j=m_{1}, \mathcal{A}_{\mathbf{n}, m_{1}}=Q_{\mathbf{n}, m_{1}}$ and (29) directly implies that

$$
\lim _{\mathbf{n} \in \Lambda}\left|\mathcal{A}_{\mathbf{n}, m_{1}}(z)\right|^{1 /\left|\mathbf{n}_{1}\right|}=\exp \left(-P_{m_{1}} V^{\bar{\mu}_{m_{1}}}(z)\right), \quad \mathcal{K} \subset \mathbb{C} \backslash \Delta_{m_{1}}
$$

For $j \in\left\{-m_{2}-1, \ldots, m_{1}-1\right\}$, using (12) and (16) with $q=Q_{\mathbf{n}, j+1}$, we obtain

$$
\mathcal{A}_{\mathbf{n}, j}(z)=\frac{Q_{\mathbf{n}, j}(z)}{Q_{\mathbf{n}, j+1}(z)} \int \frac{Q_{\mathbf{n}, j+1}^{2}(x)}{Q_{\mathbf{n}, j}(x)} \frac{\mathcal{A}_{\mathbf{n}, j+1}(x)}{Q_{\mathbf{n}, j+1}(x)} \frac{d \sigma_{j+1}(x)}{z-x},
$$


$\left(Q_{\mathbf{n},-m_{2}-1} \equiv 1\right.$.) From $(29)$, it follows that

$$
\lim _{\mathbf{n} \in \Lambda}\left|\frac{Q_{\mathbf{n}, j}(z)}{Q_{\mathbf{n}, j+1}(z)}\right|^{1 /\left|\mathbf{n}_{1}\right|}=\exp \left(P_{j+1} V^{\bar{\mu}_{j+1}}(z)-P_{j} V^{\bar{\mu}_{j}}(z)\right), \quad \mathcal{K} \subset \mathbb{C} \backslash\left(\Delta_{j} \cup \Delta_{j+1}\right)
$$

(we also use that the zeros of $Q_{\mathbf{n}, j}$ and $Q_{\mathbf{n}, j+1}$ lie in $\Delta_{j}$ and $\Delta_{j+1}$, respectively). It remains to find the $\left|\mathbf{n}_{1}\right|$-th root asymptotic behavior of the integral.

Fix a compact set $\mathcal{K} \subset \mathbb{C} \backslash \Delta_{j+1}$. It is easy to verify that (for the definition of $K_{\mathbf{n}, j+1}^{2}$ see proof of Theorem 4.3 above)

$$
\frac{C_{1}}{K_{\mathbf{n}, j+1}^{2}} \leq\left|\int \frac{Q_{\mathbf{n}, j+1}^{2}(x)}{Q_{\mathbf{n}, j}(x)} \frac{\mathcal{A}_{\mathbf{n}, j+1}(x)}{Q_{\mathbf{n}, j+1}(x)} \frac{d \sigma_{j+1}(x)}{z-x}\right| \leq \frac{C_{2}}{K_{\mathbf{n}, j+1}^{2}},
$$

where

$$
C_{1}=\frac{\min \left\{\max \{|u-x|,|v|: z=u+i v\}: z \in \mathcal{K}, x \in \Delta_{j+1}\right\}}{\max \left\{|z-x|^{2}: z \in \mathcal{K}, x \in \Delta_{j+1}\right\}}>0
$$

and

Taking into account (30)

$$
\lim _{\mathbf{n} \in \Lambda}\left|\int \frac{Q_{\mathbf{n}, j+1}^{2}(x)}{Q_{\mathbf{n}, j}(x)} \frac{\mathcal{A}_{\mathbf{n}, j+1}(x)}{Q_{\mathbf{n}, j+1}(x)} \frac{d \sigma_{j+1}(x)}{z-x}\right|^{1 /\left|\mathbf{n}_{1}\right|}=\exp \left(-2 \sum_{k=j+1}^{m_{1}} \omega_{k}^{\bar{\mu}} / P_{k}\right)
$$

From (43)-(45), we obtain (40) and we are done.

Remark 5.2. Taking into consideration that the polynomials $Q_{\mathbf{n}, j}$ (see Propositions 2.5 and 2.7) and the functions

$$
\int \frac{Q_{\mathbf{n}, j}^{2}(x)}{Q_{\mathbf{n}, j-1}(x)} \frac{\mathcal{A}_{\mathbf{n}, j}(x)}{Q_{\mathbf{n}, j}(x)} \frac{d \sigma_{j}(x)}{z-x},
$$

may have at most one zero in each of the connected components of $\Delta_{j} \backslash E_{j}$, in place of (40) one can prove convergence in capacity on each compact subset $\mathcal{K} \subset \mathbb{C} \backslash\left(E_{j} \cup E_{j+1}\right)$. More precisely, for any such compact set $\mathcal{K}$ and each $\varepsilon>0$

$$
\lim _{\mathbf{n} \in \Lambda} \operatorname{cap}\left\{z \in \mathcal{K}:\left.|| \mathcal{A}_{\mathbf{n}, j}(z)\right|^{1 /\left|\mathbf{n}_{1}\right|}-G_{j}(z) \mid>\varepsilon\right\}=0
$$

Set

$$
U_{j}^{\bar{\mu}}(z)=P_{j} V^{\bar{\mu}_{j}}(z)-P_{j+1} V^{\bar{\mu}_{j+1}}(z)+2 \sum_{k=j+1}^{m_{1}} \frac{\omega_{k}^{\bar{\mu}}}{P_{k}}, \quad j=-m_{2}-1, \ldots, m_{1}-1
$$

and

$$
U_{m_{1}}^{\bar{\mu}}(z)=P_{m_{1}} V^{\bar{\mu}_{m_{1}}(z)}
$$

Hence, $G_{j}(z)=\exp \left(-U_{j}^{\bar{\mu}}(z)\right), j=-m_{2}-1, \ldots, m_{1}$.

We have that for $j=-m_{2}, \ldots, m_{1}\left(P_{-m_{2}-1}=P_{m_{1}+1}=0\right)$

$$
\frac{P_{j}}{2}\left(U_{j}^{\bar{\mu}}(z)-U_{j-1}^{\bar{\mu}}(z)\right)=-\frac{P_{j+1} P_{j}}{2} V^{\bar{\mu}_{j+1}}(z)+P_{j}^{2} V^{\bar{\mu}_{j}}(z)-\frac{P_{j} P_{j-1}}{2} V^{\bar{\mu}_{j-1}}(z)-\omega_{j}^{\bar{\mu}} .
$$


From the equilibrium property (see Lemma 1.2 and (38)), it follows that

$$
U_{j}^{\bar{\mu}}(x)-U_{j-1}^{\bar{\mu}}(x) \begin{cases}=0, & x \in \operatorname{supp}\left(\bar{\mu}_{j}\right), \\ \geq 0, & x \in E_{j} .\end{cases}
$$

Define

$$
p_{j}= \begin{cases}p_{1, j}, & j=0, \ldots, m_{1}, \\ -p_{2,-j-1}, & j=-m_{2}-1, \ldots,-1 .\end{cases}
$$

It is easy to verify that for $j=-m_{2}, \ldots, m_{1}$

$$
U_{j}^{\bar{\mu}}(z)-U_{j-1}^{\bar{\mu}}(z)=\mathcal{O}\left(\left(p_{j}-p_{j-1}\right) \log 1 /|z|\right), \quad z \rightarrow \infty .
$$

In particular, $U_{j}^{\bar{\mu}}(z)-U_{j-1}^{\bar{\mu}}(z)=\mathcal{O}(1), z \rightarrow \infty$, whenever $p_{j}=p_{j-1}$. By assumption, $p_{j}-p_{j-1} \leq$ $0, j=-m_{2}, \ldots, m_{1}$ except for $p_{0}-p_{-1}=p_{1,0}+p_{2,0}>0$.

For all $j$, the function $U_{j}^{\bar{\mu}}-U_{j-1}^{\bar{\mu}}$ is subharmonic in $\mathbb{C} \backslash \operatorname{supp}\left(\bar{\mu}_{j}\right)$. If $p_{j} \geq p_{j-1}$, then it is subharmonic in all $\overline{\mathbb{C}} \backslash \operatorname{supp}\left(\bar{\mu}_{j}\right)$. According to what was said above, when $j=0$ or $p_{j}=p_{j-1}$, from the equilibrium condition and the maximum principle, we have that $U_{j}^{\bar{\mu}}-U_{j-1}^{\bar{\mu}} \equiv 0$ on $\operatorname{supp}\left(\sigma_{j}\right)=$ $E_{j}$ and $U_{j}^{\bar{\mu}}<U_{j-1}^{\bar{\mu}}$ on $\overline{\mathbb{C}} \backslash \operatorname{supp}\left(\sigma_{j}\right)$. In particular, in this case we have that $\operatorname{supp}\left(\bar{\mu}_{j}\right)=\operatorname{supp}\left(\sigma_{j}\right)$.

When $p_{j-1}>p_{j}$, (46) implies that in a neighborhood of $z=\infty, U_{j}^{\bar{\mu}}>U_{j-1}^{\bar{\mu}}$. Let $\gamma_{j}=\{z \in$ $\left.\mathbb{C}: U_{j}^{\bar{\mu}}(z)-U_{j-1}^{\bar{\mu}}(z)=0\right\}$. The equilibrium condition entails that $\gamma_{j} \supset \operatorname{supp}\left(\bar{\mu}_{j}\right)$ and the initial remark indicates that $\gamma_{j}$ is bounded. Consider any bounded component of the complement of $\gamma_{j}$. On it, $U_{j}^{\bar{\mu}}-U_{j-1}^{\bar{\mu}}$ is subharmonic and on its boundary $U_{j}^{\bar{\mu}}-U_{j-1}^{\bar{\mu}}=0$. Thus, on any bounded component of the complement of $\gamma_{j}$ we have that $U_{j}^{\bar{\mu}}<U_{j-1}^{\bar{\mu}}$. From the initial remark it follows that on the unbounded component of the complement of $\gamma_{j}, U_{j}^{\bar{\mu}}>U_{j-1}^{\bar{\mu}}$.

Fix $j \in\left\{0, \ldots, m_{1}\right\}$. For each $k \in\left\{j, \ldots, m_{1}\right\}$ define

$$
D_{k}^{j}:=\left\{z \in \mathbb{C} \backslash \cup_{i=j}^{m_{1}} \Delta_{i}: U_{k}^{\bar{\mu}}(z)<U_{i}^{\bar{\mu}}(z), i=j, \ldots, m_{1}, i \neq k\right\}, \quad D_{m_{1}}^{m_{1}}:=\mathbb{C} \backslash \Delta_{m_{1}} .
$$

Let

$$
\zeta_{j}(z)=\min \left\{U_{k}^{\bar{\mu}}(z): k=j, \ldots, m_{1}\right\} .
$$

Corollary 5.3. Let $\Lambda=\Lambda\left(p_{1,0}, \ldots, p_{1, m_{1}} ; p_{2,0}, \ldots, p_{2, m_{2}}\right) \subset \mathbb{Z}_{+}^{m_{1}+1}(\bullet) \times \mathbb{Z}_{+}^{m_{2}+1}(\bullet),\left(S^{1}, S^{2}\right) \in$ $\operatorname{Reg}, S^{1}=\mathcal{N}\left(\sigma_{0}^{1}, \ldots, \sigma_{m_{1}}^{1}\right), S^{2}=\mathcal{N}\left(\sigma_{0}^{2}, \ldots, \sigma_{m_{2}}^{2}\right)$ be given. Let $\left(a_{\mathbf{n}, 0}, a_{\mathbf{n}, 1}, \ldots, a_{\mathbf{n}, m_{1}}\right), \mathbf{n} \in \Lambda$, be the associated sequence of "monic" mixed type multiple orthogonal polynomials. Then, for $j=0, \ldots, m_{1}$

$$
\lim _{\mathbf{n} \in \Lambda}\left|a_{\mathbf{n}, j}(z)\right|^{1 /\left|\mathbf{n}_{1}\right|}=\exp \left(-\zeta_{j}(z)\right), \quad \mathcal{K} \subset \cup_{k=j}^{m_{1}} D_{k}^{j},
$$

and

$$
\limsup _{\mathbf{n} \in \Lambda}\left|a_{\mathbf{n}, j}(z)\right|^{1 /\left|\mathbf{n}_{1}\right|} \leq \exp \left(-\zeta_{j}(z)\right), \quad \mathcal{K} \subset \mathbb{C} \backslash \cup_{k=j}^{m_{1}} \Delta_{k} .
$$

In particular, if $p_{1,0}=\cdots=p_{1, m_{1}}=1 /\left(m_{1}+1\right)$, then

$$
\lim _{\mathbf{n} \in \Lambda}\left|a_{\mathbf{n}, j}(z)\right|^{1 /\left|\mathbf{n}_{1}\right|}=\exp \left(-U_{m_{1}}^{\bar{\mu}}(z)\right), \quad \mathcal{K} \subset \mathbb{C} \backslash \cup_{k=j}^{m_{1}} \Delta_{k} .
$$


Proof. For $j=m_{1}, \mathcal{A}_{\mathbf{n}, m_{1}}=a_{\mathbf{n}, m_{1}}, D_{m_{1}}^{m_{1}}=\mathbb{C} \backslash \Delta_{m_{1}}$ and $\zeta_{m_{1}}=U_{m_{1}}^{\bar{\mu}}$. Therefore, (47) reduces to (40) and implies (48). Let us prove these relations for $j=0, \ldots, m_{1}-1$.

The $\mathcal{A}_{\mathbf{n}, j}$ are expressed in terms of the $a_{\mathbf{n}, k}, k=j, \ldots, m_{1}$, through a linear triangular scheme of equations with function coefficients which do not depend on $\mathbf{n}$. Using this system, we can solve for $a_{\mathbf{n}, j}$, in terms of $\mathcal{A}_{\mathbf{n}, k}, k=j, \ldots, m_{1}$.

Given $j \in\left\{1, \ldots, m_{1}\right\}$ and $0 \leq i<j$, we have

$$
(-1)^{j-i}\left\langle\sigma_{i}^{1}, \ldots, \sigma_{j}^{1} \widehat{\rangle}(z)=\int \cdots \int \frac{d \sigma_{i}^{1}\left(x_{i}\right) \cdots d \sigma_{j}^{1}\left(x_{j}\right)}{\left(z-x_{i}\right)\left(x_{i+1}-x_{i}\right) \cdots\left(x_{j}-x_{j-1}\right)},\right.
$$

where $\langle\cdot \hat{\gamma}(z)$ denotes the Cauchy transform of the indicated measure, and

$$
\left\langle\sigma_{j}^{1}, \ldots, \sigma_{i}^{1} \widehat{\rangle}(z)=\int \cdots \int \frac{d \sigma_{i}^{1}\left(x_{i}\right) \cdots d \sigma_{j}^{1}\left(x_{j}\right)}{\left(x_{i+1}-x_{i}\right) \cdots\left(x_{j}-x_{j-1}\right)\left(z-x_{j}\right)} .\right.
$$

Consequently,

$$
(-1)^{j-i}\left\langle\sigma_{i}^{1}, \ldots, \sigma_{j}^{1}\right\rangle(z)-\left\langle\sigma_{j}^{1}, \ldots, \sigma_{i}^{1} \widehat{\rangle}(z)=\int \cdots \int \frac{-\left(x_{j}-x_{i}\right) d \sigma_{i}^{1}\left(x_{i}\right) \cdots d \sigma_{j}^{1}\left(x_{j}\right)}{\left(z-x_{i}\right)\left(x_{i+1}-x_{i}\right) \cdots\left(x_{j}-x_{j-1}\right)\left(z-x_{j}\right)} .\right.
$$

Since $x_{j}-x_{i}=x_{j}-x_{j-1}+x_{j-1}-\cdots-x_{i+1}+x_{i+1}-x_{i}$, substituting this in the previous formula, we obtain

$$
\left\langle\sigma_{j}^{1}, \ldots, \sigma_{i}^{1} \widehat{\rangle}(z)=\sum_{k=i}^{j-1}(-1)^{k-i}\left\langle\sigma_{i}^{1}, \ldots, \sigma_{k}^{1} \widehat{\gamma}(z)\left\langle\sigma_{j}^{1}, \ldots, \sigma_{k+1}^{1} \widehat{\gamma}(z)+(-1)^{j-i}\left\langle\sigma_{i}^{1}, \ldots, \sigma_{j}^{1} \widehat{\rangle}(z) .\right.\right.\right.\right.
$$

(This formula is applicable to any Nikishin system. We will use it on $S^{2}$ in the last section.)

Using formula (50) it is easy to deduce that (the sum is empty when $j=m_{1}$ )

$$
a_{\mathbf{n}, j}(z)=\mathcal{A}_{\mathbf{n}, j}(z)+\sum_{k=j+1}^{m_{1}}(-1)^{k-j}\left\langle\sigma_{k}^{1}, \ldots, \sigma_{j+1}^{1} \hat{\gamma}(z) \mathcal{A}_{\mathbf{n}, k}(z) .\right.
$$

Taking (40) into consideration, on $D_{k}^{j}$ the term containing $\mathcal{A}_{\mathbf{n}, k}$ dominates the sum (notice that $\left\langle\sigma_{k}^{1}, \ldots, \sigma_{j+1}^{1} \widehat{\gamma}(z) \neq 0, z \in \mathbb{C} \backslash \Delta_{k}\right)$ and (47) immediately follows. On the complement of $\cup_{k=j}^{m_{1}} D_{k}^{j}$ there is no dominating term and all we can conclude from the previous equality is (48).

Let $p_{1,0}=\cdots=p_{1, m_{1}}=1 /\left(m_{1}+1\right)$. In this case, on $\mathbb{C} \backslash \cup_{k=j}^{m_{1}} \Delta_{k}$ we have that $U_{m_{1}}^{\bar{\mu}}(z)<$ $U_{m_{1}-1}^{\bar{\mu}}(z)<\cdots<U_{j}^{\bar{\mu}}(z)$ and (49) follows from (47).

\section{RAtio ASYMPtotics}

Here, we study the convergence of the sequences $\left\{Q_{\mathbf{n}^{l}, j} / Q_{\mathbf{n}, j}\right\}, \mathbf{n} \in \Lambda \subset \mathbb{Z}_{+}^{m_{1}+1}(\bullet) \times \mathbb{Z}_{+}^{m_{2}+1}(\bullet)$ and of the ratio of the corresponding linear forms. We maintain the notation introduced in Section 4 , namely

$$
\begin{gathered}
\Delta_{j}=\Delta_{j}^{1}, \quad \sigma_{j}=\sigma_{j}^{1}, \quad j=0,1, \ldots, m_{1}, \\
\Delta_{j}=\Delta_{-j}^{2}, \quad \sigma_{j}=\sigma_{-j}^{2}, \quad j=0,-1, \ldots,-m_{2},
\end{gathered}
$$


and

$$
N_{\mathbf{n}, j}= \begin{cases}N_{1, j}(\mathbf{n})-1, & j=0,1 \ldots, m_{1} \\ N_{2,-j}(\mathbf{n}), & j=0,-1, \ldots,-m_{2}\end{cases}
$$

Set

$$
\mathcal{H}_{\mathbf{n}, j}=\frac{Q_{\mathbf{n}, j+1} \mathcal{A}_{\mathbf{n}, j}}{Q_{\mathbf{n}, j}}, \quad j=-m_{2}-1, \ldots, m_{1},
$$

$\left(Q_{\mathbf{n},-m_{2}-1} \equiv Q_{\mathbf{n}, m_{1}+1} \equiv 1\right.$ and $\left.\mathcal{H}_{\mathbf{n}, m_{1}} \equiv 1\right)$. With these notations, relations (11), (15), (12), and

(16) (replacing general $q$ by $Q_{\mathbf{n}, j+1}$ and shifting the index $j$ by -1 ) can be rewritten as follows

$$
\int x^{\nu} Q_{\mathbf{n}, j}(x) \frac{\left|\mathcal{H}_{\mathbf{n}, j}(x)\right| d\left|\sigma_{j}\right|(x)}{\left|Q_{\mathbf{n}, j-1}(x) Q_{\mathbf{n}, j+1}(x)\right|}=0, \quad \nu=0, \ldots, N_{\mathbf{n}, j}-1, \quad j=-m_{2}, \ldots, m_{1},
$$

and

$$
\mathcal{H}_{\mathbf{n}, j-1}(z)=\int \frac{Q_{\mathbf{n}, j}^{2}(x)}{z-x} \frac{\mathcal{H}_{\mathbf{n}, j}(x) d \sigma_{j}(x)}{Q_{\mathbf{n}, j-1}(x) Q_{\mathbf{n}, j+1}(x)}, \quad j=-m_{2}, \ldots, m_{1} .
$$

Since on the interval $\Delta_{j}$ the measure $\sigma_{j}$ and the functions $\mathcal{H}_{\mathbf{n}, j}, Q_{\mathbf{n}, j-1} Q_{\mathbf{n}, j+1}$, preserve a constant sign, we can take their absolute values in (52) without altering the orthogonality relations.

For each $j=-m_{2}, \ldots, m_{1}$, define

$$
K_{\mathbf{n}, j}=\left(\int_{\Delta_{j}} Q_{\mathbf{n}, j}^{2}(x) \frac{\left|\mathcal{H}_{\mathbf{n}, j}(x)\right| d\left|\sigma_{j}\right|(x)}{\left|Q_{\mathbf{n}, j-1}(x) Q_{\mathbf{n}, j+1}(x)\right|}\right)^{-1 / 2} .
$$

Take

Define

$$
q_{\mathbf{n}, j}=\kappa_{\mathbf{n}, j} Q_{\mathbf{n}, j}, \quad h_{\mathbf{n}, j-1}=K_{\mathbf{n}, j}^{2} \mathcal{H}_{\mathbf{n}, j-1}
$$

and

$$
d \rho_{\mathbf{n}, j}(x)=\frac{h_{\mathbf{n}, j}(x) d \sigma_{j}(x)}{Q_{\mathbf{n}, j-1}(x) Q_{\mathbf{n}, j+1}(x)} .
$$

From (52) and the notation introduced above, we obtain

$$
\int_{\Delta_{j}} x^{\nu} Q_{\mathbf{n}, j}(x) d\left|\rho_{\mathbf{n}, j}\right|(x)=0, \quad \nu=0, \ldots, N_{\mathbf{n}, j}-1, \quad j=-m_{2}, \ldots, m_{1},
$$

and $q_{n, j}$ is orthonormal with respect to the varying measure $\left|\rho_{\mathbf{n}, j}\right|$. On the other hand, using (53) it follows that

$$
h_{\mathbf{n}, j-1}(z)=\varepsilon_{\mathbf{n}, j} \int_{\Delta_{j}} \frac{q_{\mathbf{n}, j}^{2}(x)}{z-x} d\left|\rho_{\mathbf{n}, j}\right|(x), \quad j=-m_{2}, \ldots, m_{1},
$$

where $\varepsilon_{\mathbf{n}, j}$ denotes the sign of the varying measure $\rho_{\mathbf{n}, j}$.

The proof of Theorem 6.4 below has three steps. First, we show that for each $j \in\left\{-m_{2}, \ldots, m_{1}\right\}$ the sequence $\left\{Q_{\mathbf{n}^{l}, j} / Q_{\mathbf{n}, j}\right\}$ is uniformly bounded on each compact subset contained in $\mathbb{C} \backslash \operatorname{supp}\left(\sigma_{j}\right)$ (for all sufficiently large $\left|\mathbf{n}_{1}\right|$ ). Taking a subsequence of multi-indices such that all the sequences of ratios of polynomials have limit, we show that the limit functions must satisfy a system of 
boundary value problems. This system happens to have a unique solution from which we derive that all convergent subsequences have the same limit. Finally, we show that the limit functions can be expressed in terms of the branches of certain conformal representations of a related compact Riemann surface onto the extended complex plane.

In this section, we assume that $\operatorname{supp}\left(\sigma_{j}\right)=\widetilde{\Delta}_{j} \cup e_{j}, j=-m_{2}, \ldots, m_{1}$, where $\widetilde{\Delta}_{j}=\left[a_{j}, b_{j}\right]$ is a bounded interval of the real line, $\left|\sigma_{j}^{\prime}\right|>0$ a.e. on $\widetilde{\Delta}_{j}$, and $e_{j}$ is a set without accumulation points in $\overline{\mathbb{R}} \backslash \widetilde{\Delta}_{j}$. We denote this writing $S^{1}=\mathcal{N}^{\prime}\left(\sigma_{0}^{1}, \ldots, \sigma_{m_{1}}^{1}\right), S^{2}=\mathcal{N}^{\prime}\left(\sigma_{0}^{2}, \ldots, \sigma_{m_{2}}^{2}\right)$.

In order to fulfill the first step, Theorem 3.5 would be sufficient if $\Delta_{j}=\widetilde{\Delta}_{j}, j=-m_{2}, \ldots, m_{1}$. In order to allow the compact sets to enter the connected components of $\Delta_{j} \backslash \operatorname{supp}\left(\sigma_{j}\right)$, we need to show that the zeros falling in the intervals $I$ (see Propositions 2.5 and 2.7) are attracted to points in $\operatorname{supp}\left(\sigma_{j}\right) \backslash \widetilde{\Delta}_{j}$. In our aid comes the next result.

Lemma 6.1. Let $S^{1}=\mathcal{N}^{\prime}\left(\sigma_{0}^{1}, \ldots, \sigma_{m_{1}}^{1}\right), S^{2}=\mathcal{N}^{\prime}\left(\sigma_{0}^{2}, \ldots, \sigma_{m_{2}}^{2}\right)$ be given, and let $\Lambda \subset \mathbb{Z}_{+}^{m_{1}+1}(\bullet) \times$ $\mathbb{Z}_{+}^{m_{2}+1}(\bullet)$ be an infinite sequence of distinct multi-indices such that

$$
\sup _{\mathbf{n} \in \Lambda}\left(n_{1,0}-n_{1, m_{1}}\right)<\infty, \quad \sup _{\mathbf{n} \in \Lambda}\left(n_{2,0}-n_{1, m_{2}}\right)<\infty .
$$

For any continuous function $f$ on $\operatorname{supp}\left(\sigma_{j}\right)$

$$
\lim _{\mathbf{n} \in \Lambda} \int f(x) q_{\mathbf{n}, j}^{2}(x) d\left|\rho_{\mathbf{n}, j}\right|(x)=\frac{1}{\pi} \int_{a_{j}}^{b_{j}} f(x) \frac{d x}{\sqrt{\left(b_{j}-x\right)\left(x-a_{j}\right)}},
$$

where $\widetilde{\Delta}_{j}=\left[a_{j}, b_{j}\right],-m_{2} \leq j \leq m_{1}$. In particular,

$$
\lim _{\mathbf{n} \in \Lambda} \varepsilon_{\mathbf{n}, j} h_{\mathbf{n}, j-1}(z)=\frac{1}{\sqrt{\left(z-b_{j}\right)\left(z-a_{j}\right)}}, \quad \mathcal{K} \subset \mathbb{C} \backslash \operatorname{supp}\left(\sigma_{j}\right),
$$

where $\sqrt{\left(z-b_{j}\right)\left(z-a_{j}\right)}>0$ if $z>b_{j}$. Consequently, for $j=-m_{2}, \ldots, m_{1}$, each point of $\operatorname{supp}\left(\sigma_{j}\right) \backslash$ $\widetilde{\Delta}_{j}$ is a limit of zeros of $\left\{Q_{\mathbf{n}, j}\right\}, \mathbf{n} \in \Lambda$.

Proof. We will prove this by induction on $j$. For $j=m_{1}$, using Corollary 3 in [2] and the second condition in (59), it follows that

$$
\lim _{\mathbf{n} \in \Lambda} \int_{\Delta_{m_{1}}} f(x) q_{\mathbf{n}, m_{1}}^{2}(x) \frac{d\left|\sigma_{m_{1}}\right|(x)}{\left|Q_{\mathbf{n}, m_{1}-1}(x)\right|}=\frac{1}{\pi} \int_{\widetilde{\Delta}_{m_{1}}} f(x) \frac{d x}{\sqrt{\left(b_{m_{1}}-x\right)\left(x-a_{m_{1}}\right)}},
$$

where $f$ is continuous on $\operatorname{supp}\left(\sigma_{m_{1}}\right)$. Take $f(x)=(z-x)^{-1}$ where $z \in \mathbb{C} \backslash \operatorname{supp}\left(\sigma_{m_{1}}\right)$. According to $(58)$ and the previous limit one obtains that

$$
\lim _{\mathbf{n} \in \Lambda} \varepsilon_{\mathbf{n}, m_{1}} h_{\mathbf{n}, m_{1}-1}(z)=\frac{1}{\sqrt{\left(z-b_{m_{1}}\right)\left(z-a_{m_{1}}\right)}}=: h_{m_{1}}(z)
$$

pointwise on $\mathbb{C} \backslash \operatorname{supp}\left(\sigma_{m_{1}}\right)$. Since

$$
\left|\int_{\Delta_{m_{1}}} \frac{q_{\mathbf{n}, m_{1}}^{2}(x)}{z-x} \frac{d\left|\sigma_{m_{1}}\right|(x)}{\left|Q_{\mathbf{n}, m_{1}-1}(x)\right|}\right| \leq \frac{1}{d\left(\mathcal{K}, \operatorname{supp}\left(\sigma_{m_{1}}\right)\right)}, \quad z \in \mathcal{K} \subset \mathbb{C} \backslash \operatorname{supp}\left(\sigma_{m_{1}}\right),
$$

where $d\left(\mathcal{K}, \operatorname{supp}\left(\sigma_{m_{1}}\right)\right)$ denotes the distance between the two compact sets, the sequence $\left\{h_{\mathbf{n}, m_{1}-1}\right\}$, $\mathbf{n} \in \Lambda$, is uniformly bounded on compact subsets of $\mathbb{C} \backslash \operatorname{supp}\left(\sigma_{m_{1}}\right)$ and (61) follows for $j=m_{1}$. 
Let $\zeta \in \operatorname{supp}\left(\sigma_{m_{1}}\right) \backslash \widetilde{\Delta}_{m_{1}}$. Take $r>0$ sufficiently small so that the circle $C_{r}=\{z:|z-\zeta|=r\}$ surrounds no other point of $\operatorname{supp}\left(\sigma_{m_{1}}\right) \backslash \widetilde{\Delta}_{m_{1}}$ and contains no zero of $q_{\mathbf{n}, m_{1}}, \mathbf{n} \in \Lambda$. From (61) for $j=m_{1}$

$$
\lim _{\mathbf{n} \in \Lambda} \frac{1}{2 \pi i} \int_{C_{r}} \frac{\varepsilon_{\mathbf{n}, m_{1}} h_{\mathbf{n}, m_{1}-1}^{\prime}(z)}{\varepsilon_{\mathbf{n}, m_{1}} h_{\mathbf{n}, m_{1}-1}(z)} d z=\frac{1}{2 \pi i} \int_{C_{r}} \frac{h_{m_{1}}^{\prime}(z)}{h_{m_{1}}(z)} d z=0 .
$$

Since $\zeta$ is a mass point of $\sigma_{m_{1}}$, formula (58) indicates that either $h_{\mathbf{n}, m_{1}-1}$ has a simple pole at $\zeta$ or $Q_{\mathbf{n}, m_{1}}(\zeta)=0$. In any case, from (62) and the argument principle, it follows that for all sufficiently large $|\mathbf{n}|, \mathbf{n} \in \Lambda, Q_{\mathbf{n}, m_{1}}$ must have a simple zero inside $C_{r}$. The parameter $r$ can be taken arbitrarily small; therefore, the last statement of the lemma readily follows and the basis of induction is fulfilled.

Let us assume that the lemma is satisfied for $j \in\left\{k+1, \ldots, m_{1}\right\},-m_{2} \leq k \leq m_{1}-1$, and let us prove that it is also true for $k$. From (61) applied to $j=k+1$, we have that

$$
\lim _{\mathbf{n} \in \Lambda}\left|h_{\mathbf{n}, k}(x)\right|=\frac{1}{\sqrt{\left|\left(x-b_{k+1}\right)\left(x-a_{k+1}\right)\right|}}
$$

uniformly on $\Delta_{k} \subset \mathbb{C} \backslash \operatorname{supp}\left(\sigma_{k+1}\right)$. It follows that $\left\{\left|h_{\mathbf{n}, k}\right| d\left|\sigma_{k}\right|\right\}, \mathbf{n} \in \Lambda$, is a sequence of Denisov type measures according to Definition 3 in [2]. Additionally, $\left(\left\{\left|h_{\mathbf{n}, k}\right| d\left|\sigma_{k}\right|\right\},\left\{\left|Q_{\mathbf{n}, k-1} Q_{\mathbf{n}, k+1}\right|\right\}, l\right), \mathbf{n} \in \Lambda$, is strongly admissible as in Definition 2 of [2] for each $l \in \mathbb{Z}$ (see paragraph just after both definitions in the referred paper). Therefore, we can apply Corollary 3 in [2] of which (60) for $j=k$ is a particular case. In the proof of Corollary 3 of [2] (see also Theorem 9 in [4]) it is required that the inequality $\operatorname{deg}\left(Q_{\mathbf{n}, j-1} Q_{\mathbf{n}, j+1}\right)-2 \operatorname{deg}\left(Q_{\mathbf{n}, j}\right) \leq C$ holds for every $\mathbf{n} \in \Lambda$, where $C \geq 0$ is a constant. It is straightforward to check that this condition is satisfied under the assumption (59).

Now we return to the induction argument. From (60) for $j=k,(61)$ and the rest of the statements of the lemma immediately follow just as in the case when $j=m_{1}$. With this we conclude the proof.

Now, we are ready to prove normality for the families of ratios of the polynomials $Q_{\mathbf{n}, j}$.

Lemma 6.2. Let $S^{1}=\mathcal{N}^{\prime}\left(\sigma_{0}^{1}, \ldots, \sigma_{m_{1}}^{1}\right), S^{2}=\mathcal{N}^{\prime}\left(\sigma_{0}^{2}, \ldots, \sigma_{m_{2}}^{2}\right)$ be given, and let $\Lambda \subset \mathbb{Z}_{+}^{m_{1}+1}(\bullet) \times$ $\mathbb{Z}_{+}^{m_{2}+1}(\bullet)$ be an infinite sequence of distinct multi-indices such that

$$
\sup _{\mathbf{n} \in \Lambda}\left(n_{1,0}-n_{1, m_{1}}\right)<\infty, \quad \sup _{\mathbf{n} \in \Lambda}\left(n_{2,0}-n_{1, m_{2}}\right)<\infty .
$$

Let us assume that there exists $l=\left(l_{1} ; l_{2}\right), 0 \leq l_{1} \leq m_{1}, 0 \leq l_{2} \leq m_{2}$, such that for all $\mathbf{n} \in \Lambda$ we have that $\mathbf{n}^{l} \in \mathbb{Z}_{+}^{m_{1}+1}(\bullet) \times \mathbb{Z}_{+}^{m_{2}+1}(\bullet)$. Then, for each $j=-m_{2}, \ldots, m_{1}$, and each compact set $\mathcal{K} \subset \mathbb{C} \backslash \operatorname{supp}\left(\sigma_{j}\right)$ there exist positive constants $C_{j, 1}(\mathcal{K}), C_{j, 2}(\mathcal{K})$ such that

$$
C_{j, 1}(\mathcal{K}) \leq \inf _{z \in \mathcal{K}}\left|\frac{Q_{\mathbf{n}^{l}, j}(z)}{Q_{\mathbf{n}, j}(z)}\right| \leq \sup _{z \in \mathcal{K}}\left|\frac{Q_{\mathbf{n}^{l}, j}(z)}{Q_{\mathbf{n}, j}(z)}\right| \leq C_{j, 2}(\mathcal{K}),
$$

for all sufficiently large $\left|\mathbf{n}_{1}\right|, \mathbf{n} \in \Lambda$. 
Proof. The uniform bound from above and below on each fixed compact subset $\mathcal{K} \subset \mathbb{C} \backslash \Delta_{j}$ (for all $\mathbf{n} \in \Lambda$ ) is a direct consequence of the interlacing property of the zeros of $Q_{\mathbf{n}^{l}, j}$ and $Q_{\mathbf{n}, j}$. In fact, comparing distances to $z \in \mathcal{K}$ of consecutive interlacing zeros, it is easy to verify that

$$
\min \left\{d_{1}, \frac{d_{1}}{d_{2}}\right\} \leq \inf _{z \in \mathcal{K}}\left|\frac{Q_{\mathbf{n}^{l}, j}(z)}{Q_{\mathbf{n}, j}(z)}\right| \leq \sup _{z \in \mathcal{K}}\left|\frac{Q_{\mathbf{n}^{l}, j}(z)}{Q_{\mathbf{n}, j}(z)}\right| \leq \frac{\max \left\{d_{2}, d_{2}^{2}\right\}}{d_{1}},
$$

where $d_{2}$ denotes the diameter of $\mathcal{K} \cup \Delta_{j}$ and $d_{1}$ denotes the distance between $\mathcal{K}$ and $\Delta_{j}$. So, for such compact sets the assertion has been proved.

The additional restrictions made in the lemma guarantee that the zeros of the polynomials $Q_{\mathbf{n}^{l}, j}$ and $Q_{\mathbf{n}, j}$ lying in $\Delta_{j} \backslash \operatorname{supp}\left(\sigma_{j}\right)$ converge to the mass points as results from Lemma 6.1. Let $\mathcal{K} \subset \mathbb{C} \backslash \operatorname{supp}\left(\sigma_{j}\right)$ and suppose that $\mathcal{K} \cap \Delta_{j} \neq \emptyset$. Notice that $\mathcal{K}$ can intersect at most a finite number of open intervals $I_{1}, \ldots, I_{M}$ forming the connected components of $\Delta_{j} \backslash \operatorname{supp}\left(\sigma_{j}\right)$. The polynomials $Q_{\mathbf{n}^{l}, j}$ and $Q_{\mathbf{n}, j}$ can have at most one zero in each of those intervals. Consequently, for all $\left|\mathbf{n}_{1}\right|, \mathbf{n} \in \Lambda$, sufficiently large, the zeros of $Q_{\mathbf{n}^{l}, j}$ and $Q_{\mathbf{n}, j}$ lie at a positive distance $\varepsilon$ from $\mathcal{K}$. Now, it is easy to show that for all sufficiently large $\left|\mathbf{n}_{1}\right|$

$$
\min \left\{\varepsilon, \frac{\varepsilon}{d_{2}}\right\} \leq \inf _{z \in \mathcal{K}}\left|\frac{Q_{\mathbf{n}^{l}, j}(z)}{Q_{\mathbf{n}, j}(z)}\right| \leq \sup _{z \in \mathcal{K}}\left|\frac{Q_{\mathbf{n}^{l}, j}(z)}{Q_{\mathbf{n}, j}(z)}\right| \leq \frac{\max \left\{d_{2}, d_{2}^{2}\right\}}{\varepsilon} .
$$

This concludes the proof.

From Lemma 6.2 we know that the sequences

$$
\left\{Q_{\mathbf{n}^{l}, j} / Q_{\mathbf{n}, j}\right\}_{\mathbf{n} \in \Lambda}, \quad j=-m_{2}, \ldots, m_{1},
$$

are uniformly bounded on each compact subset of $\mathbb{C} \backslash \operatorname{supp}\left(\sigma_{j}\right)$ for all sufficiently large $\left|\mathbf{n}_{1}\right|$. By Montel's theorem, there exists a subsequence of multi-indices $\Lambda^{\prime} \subset \Lambda$ and a collection of functions $\widetilde{F}_{j}^{(l)}$, holomorphic in $\mathbb{C} \backslash \operatorname{supp}\left(\sigma_{j}\right)$, such that

$$
\lim _{\mathbf{n} \in \Lambda^{\prime}} \frac{Q_{\mathbf{n}^{l}, j}(z)}{Q_{\mathbf{n}, j}(z)}=\widetilde{F}_{j}^{(l)}(z), \quad \mathcal{K} \subset \mathbb{C} \backslash \operatorname{supp}\left(\sigma_{j}\right), \quad j=-m_{2}, \ldots, m_{1} .
$$

In principle, the functions $\widetilde{F}_{j}^{(l)}$ may depend on $\Lambda^{\prime}$. We shall see that this is not the case and, therefore, the limit in (63) holds for $\mathbf{n} \in \Lambda$. First, let us obtain some general information on the functions $\widetilde{F}_{j}^{(l)}$.

The points in $\operatorname{supp}\left(\sigma_{j}\right) \backslash \widetilde{\Delta}_{j}$ are isolated singularities of $\widetilde{F}_{j}^{(l)} . \quad$ Let $\zeta \in \operatorname{supp}\left(\sigma_{j}\right) \backslash \widetilde{\Delta}_{j}$. By Lemma $6.1, \zeta$ is a limit of zeros of $Q_{\mathbf{n}, j}$ and $Q_{\mathbf{n}^{l}, j}$ as $\left|\mathbf{n}_{1}\right| \rightarrow \infty, \mathbf{n} \in \Lambda$, and in a sufficiently small neighborhood of $\zeta$, for large $\left|\mathbf{n}_{1}\right|, \mathbf{n} \in \Lambda$, there can be at most one zero of these polynomials (so there is exactly one, for all sufficiently large $\left.\left|\mathbf{n}_{1}\right|\right)$. Let $\lim _{\mathbf{n} \in \Lambda} \zeta_{\mathbf{n}}=\zeta$ where $Q_{\mathbf{n}, j}\left(\zeta_{\mathbf{n}}\right)=0$. From formula (63)

$$
\lim _{\mathbf{n} \in \Lambda^{\prime}} \frac{\left(z-\zeta_{\mathbf{n}}\right) Q_{\mathbf{n}^{l}, j}(z)}{Q_{\mathbf{n}, j}(z)}=(z-\zeta) \widetilde{F}_{j}^{(l)}(z), \quad \mathcal{K} \subset\left(\mathbb{C} \backslash \operatorname{supp}\left(\sigma_{j}\right)\right) \cup\{\zeta\},
$$

and $(z-\zeta) \widetilde{F}_{j}^{(l)}(z)$ is analytic in a neighborhood of $\zeta$. Hence $\zeta$ is not an essential singularity of $\widetilde{F}_{j}^{(l)}$. Taking into consideration that $Q_{\mathbf{n}^{l}, j}, \mathbf{n} \in \Lambda$, also has a sequence of zeros converging to $\zeta$, from the 
argument principle it follows that $\zeta$ is a removable singularity of $\widetilde{F}_{j}^{(l)}$ which is not a zero. By Lemma 6.2 we also know that the sequence of functions $\left|Q_{\mathbf{n}^{l}, j} / Q_{\mathbf{n}, j}\right|, \mathbf{n} \in \Lambda$, is uniformly bounded from below by a positive constant for all sufficiently large $\left|\mathbf{n}_{1}\right|$. Therefore, in $\mathbb{C} \backslash \operatorname{supp}\left(\sigma_{j}\right)$ the function $\widetilde{F}_{j}^{(l)}$ is also different from zero. According to the definitions of $Q_{\mathbf{n}, j}, Q_{\mathbf{n}^{l}, j}$, and Propositions 2.5 and 2.7 (see also (51)), when $-l_{2} \leq j \leq l_{1}$, we have that $\operatorname{deg} Q_{\mathbf{n}^{l}, j}=N_{\mathbf{n}^{l}, j}=N_{\mathbf{n}, j}+1=\operatorname{deg} Q_{\mathbf{n}, j}+1$ whereas, for $j \in\left\{-m_{2}, \ldots,-l_{2}-1\right\} \cup\left\{l_{1}+1, \ldots, m_{1}\right\}$, we obtain that $\operatorname{deg} Q_{\mathbf{n}^{l}, j}=N_{\mathbf{n}^{l}, j}=N_{\mathbf{n}, j}=$ $\operatorname{deg} Q_{\mathbf{n}, j}$. Consequently, when $-l_{2} \leq j \leq l_{1}$, the function $\widetilde{F}_{j}^{(l)}$ has a simple pole at infinity and $\left(\widetilde{F}_{j}^{(l)}\right)^{\prime}(\infty)=1$, whereas, for $j \in\left\{-m_{2}, \ldots,-l_{2}-1\right\} \cup\left\{l_{1}+1, \ldots, m_{1}\right\}$, it is analytic at infinity and $\widetilde{F}_{j}^{(l)}(\infty)=1$.

Now, let us prove that the functions $\widetilde{F}_{j}^{(l)}, j=-m_{2}, \ldots, m_{1}$, satisfy a system of boundary value problems.

Lemma 6.3. Let $S^{1}=\mathcal{N}^{\prime}\left(\sigma_{0}^{1}, \ldots, \sigma_{m_{1}}^{1}\right), S^{2}=\mathcal{N}^{\prime}\left(\sigma_{0}^{2}, \ldots, \sigma_{m_{2}}^{2}\right)$ be given, and let $\Lambda \subset \mathbb{Z}_{+}^{m_{1}+1}(\bullet) \times$ $\mathbb{Z}_{+}^{m_{2}+1}(\bullet)$ be an infinite sequence of distinct multi-indices such that

$$
\sup _{\mathbf{n} \in \Lambda}\left(n_{1,0}-n_{1, m_{1}}\right)<\infty, \quad \sup _{\mathbf{n} \in \Lambda}\left(n_{2,0}-n_{1, m_{2}}\right)<\infty .
$$

Let us assume that there exists $l=\left(l_{1} ; l_{2}\right), 0 \leq l_{1} \leq m_{1}, 0 \leq l_{2} \leq m_{2}$, such that for all $\mathbf{n} \in \Lambda$ we have that $\mathbf{n}^{l} \in \mathbb{Z}_{+}^{m_{1}+1}(\bullet) \times \mathbb{Z}_{+}^{m_{2}+1}(\bullet)$. Then, there exists a normalization $F_{j}^{(l)}, j=-m_{2}, \ldots, m_{1}$, by positive constants, of the functions $\widetilde{F}_{j}^{(l)}$ given in (63), which verifies the system of boundary value problems

$$
\begin{array}{ll}
\text { 1) } & F_{j}^{(l)}, 1 / F_{j}^{(l)} \in H\left(\mathbb{C} \backslash \widetilde{\Delta}_{j}\right), \\
\text { 2) } & \left(F_{j}^{(l)}\right)^{\prime}(\infty)>0, \quad j \in\left\{-l_{2}, \ldots, l_{1}\right\}, \\
\left.2^{\prime}\right) & F_{j}^{(l)}(\infty)>0, \quad j \in\left\{-m_{2}, \ldots,-l_{2}-1\right\} \cup\left\{l_{1}+1, \ldots, m_{1}\right\}, \\
\text { 3) } & \left|F_{j}^{(l)}(x)\right|^{2} \frac{1}{\left|\left(F_{j-1}^{(l)} F_{j+1}^{(l)}\right)(x)\right|}=1, x \in \widetilde{\Delta}_{j},
\end{array}
$$

where $F_{-m_{2}-1}^{(l)} \equiv F_{m_{1}+1}^{(l)} \equiv 1$.

Proof. The assertions 1), 2), and 2') were proved above for the functions $\widetilde{F}_{j}^{(l)}$. Consequently, they are satisfied by any normalization of these functions by means of positive constants.

From (57) applied to $\mathbf{n}$ and $\mathbf{n}^{l}$, for each $j=-m_{2}, \ldots, m_{1}$, we have

$$
\int x^{\nu} Q_{\mathbf{n}, j}(x) d\left|\rho_{\mathbf{n}, j}\right|(x)=0, \quad \nu=0, \ldots, N_{\mathbf{n}, j}-1,
$$

and

$$
\int x^{\nu} Q_{\mathbf{n}^{l}, j}(x) g_{\mathbf{n}, j}(x) d\left|\rho_{\mathbf{n}, j}\right|(x)=0, \quad \nu=0, \ldots, N_{\mathbf{n}^{l}, j}-1
$$

where

$$
g_{\mathbf{n}, j}(x)=\frac{\left|Q_{\mathbf{n}, j-1}(x) Q_{\mathbf{n}, j+1}(x)\right|}{\left|Q_{\mathbf{n}^{l}, j-1}(x) Q_{\mathbf{n}^{l}, j+1}(x)\right|} \frac{\left|h_{\mathbf{n}^{l}, j}(x)\right|}{\left|h_{\mathbf{n}, j}(x)\right|}, \quad d \rho_{\mathbf{n}, j}(x)=\frac{h_{\mathbf{n}, j}(x) d \sigma_{j}(x)}{Q_{\mathbf{n}, j-1}(x) Q_{\mathbf{n}, j+1}(x)} .
$$


From (61) and (63)

$$
\lim _{\mathbf{n} \in \Lambda^{\prime}} g_{\mathbf{n}, j}(x)=\left|\left(\widetilde{F}_{j-1}^{(l)} \widetilde{F}_{j+1}^{(l)}\right)(x)\right|^{-1}
$$

uniformly on $\Delta_{j}$.

Fix $j \in\left\{-m_{2}, \ldots,-l_{2}-1\right\} \cup\left\{l_{1}+1, \ldots, m_{1}\right\}$. As mentioned above, for this selection of $j$ we have that $\operatorname{deg} Q_{\mathbf{n}^{l}, j}=\operatorname{deg} Q_{\mathbf{n}, j}=N_{\mathbf{n}, j}$. Due to (65) and (63), from Theorems 1 and 2 of [2], it follows that

$$
\lim _{\mathbf{n} \in \Lambda^{\prime}} \frac{Q_{\mathbf{n}^{l}, j}(z)}{Q_{\mathbf{n}, j}(z)}=\frac{S_{j}(z)}{S_{j}(\infty)}=\widetilde{S}_{j}(z)=\widetilde{F}_{j}^{(l)}(z), \quad \mathcal{K} \subset \overline{\mathbb{C}} \backslash \operatorname{supp}\left(\sigma_{j}\right),
$$

where $S_{j}$ is the Szegö function on $\overline{\mathbb{C}} \backslash \widetilde{\Delta}_{j}$ with respect to $\left|\widetilde{F}_{j-1}^{(l)}(x) \widetilde{F}_{j+1}^{(l)}(x)\right|^{-1}, x \in \widetilde{\Delta}_{j}$. The function $S_{j}$ is uniquely determined by

$$
\begin{array}{ll}
\text { 1) } & S_{j}, 1 / S_{j} \in H\left(\overline{\mathbb{C}} \backslash \widetilde{\Delta}_{j}\right), \\
\text { 2) } & S_{j}(\infty)>0, \\
\text { 3) } & \left|S_{j}(x)\right|^{2} \frac{1}{\left|\left(\widetilde{F}_{j-1}^{(l)} \widetilde{F}_{j+1}^{(l)}\right)(x)\right|}=1, \quad x \in \widetilde{\Delta}_{j} .
\end{array}
$$

Now, fix $j \in\left\{-l_{2}, \ldots, l_{1}\right\}$. In this situation $\operatorname{deg} Q_{\mathbf{n}^{l}, j}=\operatorname{deg} Q_{\mathbf{n}, j}+1=N_{\mathbf{n}, j}+1$. Let $Q_{\mathbf{n}, j}^{*}(x)$ be the monic polynomial of degree $N_{\mathbf{n}, j}$ orthogonal with respect to the varying measure $g_{\mathbf{n}, j} d\left|\rho_{\mathbf{n}, j}\right|$. Using the same arguments as above, we have

$$
\lim _{\mathbf{n} \in \Lambda^{\prime}} \frac{Q_{\mathbf{n}, j}^{*}(z)}{Q_{\mathbf{n}, j}(z)}=\frac{S_{j}(z)}{S_{j}(\infty)}=\widetilde{S}_{j}(z), \quad \mathcal{K} \subset \overline{\mathbb{C}} \backslash \operatorname{supp}\left(\sigma_{j}\right) .
$$

On the other hand, since $\operatorname{deg} Q_{\mathbf{n}^{l}, j}=\operatorname{deg} Q_{\mathbf{n}, j}^{*}+1$ and both of these polynomials are orthogonal with respect to the same varying weight, by Theorem 1 of [2] and (61), it follows that

$$
\lim _{\mathbf{n} \in \Lambda^{\prime}} \frac{Q_{\mathbf{n}^{l}, j}(z)}{Q_{\mathbf{n}, j}^{*}(z)}=\frac{\varphi_{j}(z)}{\varphi_{j}^{\prime}(\infty)}=\widetilde{\varphi}_{j}(z), \quad \mathcal{K} \subset \mathbb{C} \backslash \operatorname{supp}\left(\sigma_{j}\right),
$$

where $\varphi_{j}$ denotes the conformal representation of $\overline{\mathbb{C}} \backslash \widetilde{\Delta}_{j}$ onto $\{w:|w|>1\}$ such that $\varphi_{j}(\infty)=\infty$ and $\varphi_{j}^{\prime}(\infty)>0$. The function $\varphi_{j}$ is uniquely determined by

$$
\begin{array}{ll}
\text { 1) } & \varphi_{j}, 1 / \varphi_{j} \in H\left(\mathbb{C} \backslash \widetilde{\Delta}_{j}\right), \\
\text { 2) } & \varphi_{j}^{\prime}(\infty)>0, \\
\text { 3) } & \left|\varphi_{j}(x)\right|=1, \quad x \in \widetilde{\Delta}_{j} .
\end{array}
$$

From (63), (68), and (69), we obtain

$$
\lim _{\mathbf{n} \in \Lambda^{\prime}} \frac{Q_{\mathbf{n}^{l}, j}(z)}{Q_{\mathbf{n}, j}(z)}=\left(\widetilde{S}_{j} \widetilde{\varphi}_{j}\right)(z)=\widetilde{F}_{j}^{(l)}(z), \quad \mathcal{K} \subset \mathbb{C} \backslash \operatorname{supp}\left(\sigma_{j}\right) .
$$

Thus,

$$
\widetilde{F}_{j}^{(l)}= \begin{cases}\widetilde{S}_{j} \widetilde{\varphi}_{j}, & j \in\left\{-l_{2}, \ldots, l_{1}\right\} \\ \widetilde{S}_{j}, & j \in\left\{-m_{2}, \ldots,-l_{2}-1\right\} \cup\left\{l_{1}+1, \ldots, m_{1}\right\},\end{cases}
$$


and from (67) and (72) it follows that

$$
\left|\widetilde{F}_{j}^{(l)}(x)\right|^{2} \frac{1}{\left|\left(\widetilde{F}_{j-1}^{(l)} \widetilde{F}_{j+1}^{(l)}\right)(x)\right|}=\frac{1}{\omega_{j}}, \quad x \in \widetilde{\Delta}_{j}, \quad j=-m_{2}, \ldots, m_{1},
$$

where

$$
\omega_{j}= \begin{cases}\left(S_{j} \varphi_{j}^{\prime}\right)^{2}(\infty), & j \in\left\{-l_{2}, \ldots, l_{1}\right\} \\ S_{j}^{2}(\infty), & j \in\left\{-m_{2}, \ldots,-l_{2}-1\right\} \cup\left\{l_{1}+1, \ldots, m_{1}\right\}\end{cases}
$$

Now, let us show that there exist positive constants $c_{j}, j=-m_{2}, \ldots, m_{1}$, such that the functions $F_{j}^{(l)}=c_{j} \widetilde{F}_{j}^{(l)}$ satisfy (64). In fact, according to (73) for any such constants $c_{j}$ we have that

$$
\left|F_{j}^{(l)}(x)\right|^{2} \frac{1}{\left|\left(F_{j-1}^{(l)} F_{j+1}^{(l)}\right)(x)\right|}=\frac{c_{j}^{2}}{c_{j-1} c_{j+1} \omega_{j}}, \quad x \in \widetilde{\Delta}_{j}, \quad j=-m_{2}, \ldots, m_{1},
$$

where $c_{-m_{2}-1}=c_{m_{1}+1}=1$. The problem reduces to finding appropriate constants $c_{j}$ such that

$$
\frac{c_{j}^{2}}{c_{j-1} c_{j+1} \omega_{j}}=1, \quad j=-m_{2}, \ldots, m_{1} .
$$

Taking logarithm, we obtain the linear system of equations

$$
2 \log c_{j}-\log c_{j-1}-\log c_{j+1}=\log \omega_{j}, \quad j=-m_{2}, \ldots, m_{1}
$$

$\left(c_{-m_{2}-1}=c_{m_{1}+1}=1\right)$ on the unknowns $\log c_{j}$. This system has a unique solution with which we conclude the proof.

Consider the $\left(m_{1}+m_{2}+2\right)$-sheeted Riemann surface

$$
\mathcal{R}=\bigcup_{k=-m_{2}-1}^{m_{1}} \mathcal{R}_{k}
$$

formed by the consecutively "glued" sheets

$$
\mathcal{R}_{-m_{2}-1}:=\overline{\mathbb{C}} \backslash \widetilde{\Delta}_{-m_{2}}, \quad \mathcal{R}_{k}:=\overline{\mathbb{C}} \backslash\left(\widetilde{\Delta}_{k} \cup \widetilde{\Delta}_{k+1}\right), k=-m_{2}, \ldots, m_{1}-1, \quad \mathcal{R}_{m_{1}}:=\overline{\mathbb{C}} \backslash \widetilde{\Delta}_{m_{1}},
$$

where the upper and lower banks of the slits of two neighboring sheets are identified. Fix $l=$ $\left(l_{1}, l_{2}\right), 0 \leq l_{1} \leq m_{1}, 0 \leq l_{2} \leq m_{2}$. Let $\psi^{(l)}$ be a singled valued function defined on $\mathcal{R}$ onto the extended complex plane satisfying

$$
\begin{gathered}
\psi^{(l)}(z)=\frac{C_{1}}{z}+\mathcal{O}\left(\frac{1}{z^{2}}\right), \quad z \rightarrow \infty^{\left(-l_{2}-1\right)}, \\
\psi^{(l)}(z)=C_{2} z+\mathcal{O}(1), \quad z \rightarrow \infty^{\left(l_{1}\right)},
\end{gathered}
$$

where $C_{1}$ and $C_{2}$ are nonzero constants. Since the genus of $\mathcal{R}$ is zero, $\psi^{(l)}$ exists and is uniquely determined up to a multiplicative constant. Consider the branches of $\psi^{(l)}$, corresponding to the different sheets $k=-m_{2}-1, \ldots, m_{1}$ of $\mathcal{R}$

$$
\psi^{(l)}:=\left\{\psi_{k}^{(l)}\right\}_{k=-m_{2}-1}^{m_{1}}
$$


We normalize $\psi^{(l)}$ so that

$$
\prod_{k=-m_{2}-1}^{m_{1}}\left|\psi_{k}^{(l)}(\infty)\right|=1, \quad C_{1} \in \mathbb{R} \backslash\{0\} .
$$

Certainly, there are two $\psi^{(l)}$ verifying this normalization. Since the product of all the branches $\prod_{k=-m_{2}-1}^{m_{1}} \psi_{k}^{(l)}$ is a single valued analytic function in $\overline{\mathbb{C}}$ without singularities, by Liouville's theorem it is constant and because of the normalization introduced above this constant is either 1 or -1 .

If $\psi^{(l)}$ is such that $C_{1} \in \mathbb{R} \backslash\{0\}$, then

$$
\psi^{(l)}(z)=\overline{\psi^{(l)}(\bar{z})}, \quad z \in \mathcal{R} .
$$

In fact, let $\phi(z):=\overline{\psi^{(l)}(\bar{z})} . \phi$ and $\psi^{(l)}$ have the same divisor; consequently, there exists a constant $C$ such that $\phi=C \psi^{(l)}$. Comparing the leading coefficients of the Laurent expansion of these functions at $\infty^{\left(-l_{2}-1\right)}$, we conclude that $C=1$ since $C_{1} \in \mathbb{R} \backslash\{0\}$.

In terms of the branches of $\psi^{(l)}$, the symmetry formula above means that for each $k=-m_{2}-$ $1, \ldots, m_{1}$

$$
\psi_{k}^{(l)}: \overline{\mathbb{R}} \backslash\left(\widetilde{\Delta}_{k} \cup \widetilde{\Delta}_{k+1}\right) \longrightarrow \overline{\mathbb{R}}
$$

$\left(\widetilde{\Delta}_{-m_{2}-1}=\widetilde{\Delta}_{m_{1}+1}=\emptyset\right)$; therefore, the coefficients (in particular, the leading one) of the Laurent expansion at $\infty$ of these branches are real numbers, and

$$
\psi_{k}^{(l)}\left(x_{ \pm}\right)=\overline{\psi_{k}^{(l)}\left(x_{\mp}\right)}=\overline{\psi_{k+1}^{(l)}\left(x_{ \pm}\right)}, \quad x \in \widetilde{\Delta}_{k+1}
$$

Given an arbitrary function $F(z)$ which has in a neighborhood of infinity a Laurent expansion of the form $F(z)=C z^{k}+\mathcal{O}\left(z^{k-1}\right), C \neq 0$, and $k \in \mathbb{Z}$, we denote

$$
\widetilde{F}:=F / C
$$

$C$ is called the leading coefficient of $F$. When $C \in \mathbb{R}, \operatorname{sg}(F(\infty))$ will represent the sign of $C$.

We are ready to state and prove one of the main results of this section.

Theorem 6.4. Let $S^{1}=\mathcal{N}^{\prime}\left(\sigma_{0}^{1}, \ldots, \sigma_{m_{1}}^{1}\right), S^{2}=\mathcal{N}^{\prime}\left(\sigma_{0}^{2}, \ldots, \sigma_{m_{2}}^{2}\right)$ be given, and let $\mathbf{n} \in \Lambda \subset$ $\mathbb{Z}_{+}^{m_{1}+1}(\bullet) \times \mathbb{Z}_{+}^{m_{2}+1}(\bullet)$ be an infinite sequence of distinct multi-indices such that

$$
\sup _{\mathbf{n} \in \Lambda}\left(n_{1,0}-n_{1, m_{1}}\right)<\infty, \quad \sup _{\mathbf{n} \in \Lambda}\left(n_{2,0}-n_{1, m_{2}}\right)<\infty .
$$

Let us assume that there exists $l=\left(l_{1} ; l_{2}\right), 0 \leq l_{1} \leq m_{1}, 0 \leq l_{2} \leq m_{2}$, such that for all $\mathbf{n} \in \Lambda$ we have that $\mathbf{n}^{l} \in \mathbb{Z}_{+}^{m_{1}+1}(\bullet) \times \mathbb{Z}_{+}^{m_{2}+1}(\bullet)$. Let $\left\{Q_{\mathbf{n}, j}\right\}_{j=-m_{2}}^{m_{1}}, \mathbf{n} \in \Lambda$, be the corresponding sequences of polynomials defined in section 2. Then, for each fixed $j \in\left\{-m_{2}, \ldots, m_{1}\right\}$, we have

$$
\lim _{\mathbf{n} \in \Lambda} \frac{Q_{\mathbf{n}^{l}, j}(z)}{Q_{\mathbf{n}, j}(z)}=\widetilde{F}_{j}^{(l)}(z), \quad z \in \mathcal{K} \subset \mathbb{C} \backslash \operatorname{supp}\left(\sigma_{j}\right)
$$

where the functions satisfying (64) are

$$
F_{j}^{(l)}:=s g\left(\prod_{k=j}^{m_{1}} \psi_{k}^{(l)}(\infty)\right) \prod_{k=j}^{m_{1}} \psi_{k}^{(l)} .
$$


Proof. Since the families of functions

$$
\left\{Q_{\mathbf{n}^{l}, j} / Q_{\mathbf{n}, j}\right\}_{\mathbf{n} \in \Lambda}, \quad j=-m_{2}, \ldots, m_{1},
$$

are uniformly bounded on each compact subset $\mathcal{K} \subset \mathbb{C} \backslash \operatorname{supp}\left(\sigma_{j}\right)$ for all sufficiently large $\left|\mathbf{n}_{1}\right|, \mathbf{n} \in$ $\Lambda$, uniform convergence on compact subsets of the indicated region follows from proving that any convergent subsequence has the same limit. According to Lemma 6.3 the limit functions, appropriately normalized, of a convergent subsequence satisfy the same system of boundary value problems (64). According to Lemma 4.2 in [1] this system has a unique solution.

It remains to show that the functions defined in (80) satisfy (64). When multiplying two consecutive branches, the singularities on the common slit cancel out by the Schwarz reflection principle; therefore, 1) takes place since only the singularities of $\psi_{j}^{(l)}$ on $\widetilde{\Delta}_{j}$ remain. From the definition of $\psi^{(l)}$ it also follows that for $j=-l_{2}, \ldots, l_{1}, F_{j}^{(l)}$ has at infinity a simple pole, whereas it is regular and different from zero at infinity when $j \in\left\{-m_{2}, \ldots,-l_{2}-1\right\} \cup\left\{l_{1}+1, \ldots, m_{1}\right\}$. The factor sign in front of (80) guarantees the positivity claimed in 2) and $2^{\prime}$ ).

In order to verify 3$)$, notice that $F_{j}^{(l)} / F_{j-1}^{(l)}=\operatorname{sg}\left(\psi_{j-1}^{(l)}(\infty)\right) / \psi_{j-1}^{(l)}$. Therefore, if $j=-m_{2}+$ $1, \ldots, m_{1}$

$$
\frac{\left|F_{j}^{(l)}(x)\right|^{2}}{\left|F_{j-1}^{(l)}(x) F_{j+1}^{(l)}(x)\right|}=\frac{\left|\psi_{j}^{(l)}(x)\right|}{\left|\psi_{j-1}^{(l)}(x)\right|}=1, \quad x \in \widetilde{\Delta}_{j}
$$

on account of (78). For $j=-m_{2}$, from the definition and (78)

$$
\frac{\left|F_{-m_{2}}^{(l)}(x)\right|^{2}}{\left|F_{-m_{2}+1}^{(l)}(x)\right|}=\left|\psi_{-m_{2}}^{(l)}(x)\right|\left|\prod_{k=-m_{2}}^{m_{1}} \psi_{k}^{(l)}(x)\right|=\left|\prod_{k=-m_{2}-1}^{m_{1}} \psi_{k}^{(l)}(x)\right|=1, \quad x \in \widetilde{\Delta}_{-m_{2}},
$$

since $\prod_{k=-m_{2}-1}^{m_{1}} \psi_{k}^{(l)}$ is constantly equal to 1 or -1 on all $\overline{\mathbb{C}}$. The proof is complete.

The following corollary complements Theorem 6.4. The proof is similar to that of Corollary 4.1 in $[1]$.

Corollary 6.5. Let $S^{1}=\mathcal{N}^{\prime}\left(\sigma_{0}^{1}, \ldots, \sigma_{m_{1}}^{1}\right), S^{2}=\mathcal{N}^{\prime}\left(\sigma_{0}^{2}, \ldots, \sigma_{m_{2}}^{2}\right)$ be given, and let $\Lambda \subset \mathbb{Z}_{+}^{m_{1}+1}(\bullet) \times$ $\mathbb{Z}_{+}^{m_{2}+1}(\bullet)$ be an infinite sequence of distinct multi-indices such that

$$
\sup _{\mathbf{n} \in \Lambda}\left(n_{1,0}-n_{1, m_{1}}\right)<\infty, \quad \sup _{\mathbf{n} \in \Lambda}\left(n_{2,0}-n_{1, m_{2}}\right)<\infty .
$$

Let us assume that there exists $l=\left(l_{1} ; l_{2}\right), 0 \leq l_{1} \leq m_{1}, 0 \leq l_{2} \leq m_{2}$, such that for all $\mathbf{n} \in \Lambda$ we have that $\mathbf{n}^{l} \in \mathbb{Z}_{+}^{m_{1}+1}(\bullet) \times \mathbb{Z}_{+}^{m_{2}+1}(\bullet)$. Let $\left\{q_{\mathbf{n}, j}=\kappa_{\mathbf{n}, j} Q_{\mathbf{n}, j}\right\}_{j=-m_{2}}^{m_{1}}, \mathbf{n} \in \Lambda$, be the system of orthonormal polynomials as defined in (55) and $\left\{K_{\mathbf{n}, j}\right\}_{j=-m_{2}}^{m_{1}}, \mathbf{n} \in \Lambda$, the values given by (54). Then, for each fixed $j=-m_{2}, \ldots, m_{1}$, we have

$$
\begin{gathered}
\lim _{\mathbf{n} \in \Lambda} \frac{\kappa_{\mathbf{n}^{l}, j}}{\kappa_{\mathbf{n}, j}}=\kappa_{j}^{(l)}, \\
\lim _{\mathbf{n} \in \Lambda} \frac{K_{\mathbf{n}^{l}, j}}{K_{\mathbf{n}, j}}=\kappa_{j}^{(l)} \cdots \kappa_{m_{1}}^{(l)},
\end{gathered}
$$


and

$$
\lim _{\mathbf{n} \in \Lambda} \frac{q_{\mathbf{n}^{l}, j}(z)}{q_{\mathbf{n}, j}(z)}=\kappa_{j}^{(l)} \widetilde{F}_{j}^{(l)}(z), \quad \mathcal{K} \subset \mathbb{C} \backslash \operatorname{supp}\left(\sigma_{j}\right)
$$

where

$$
\kappa_{j}^{(l)}=\frac{c_{j}^{(l)}}{\sqrt{c_{j-1}^{(l)} c_{j+1}^{(l)}}}, \quad c_{j}^{(l)}= \begin{cases}\left(F_{j}^{(l)}\right)^{\prime}(\infty), & j \in\left\{-l_{2}, \ldots, l_{1}\right\} \\ F_{j}^{(l)}(\infty), & j \notin\left\{-l_{2}, \ldots, l_{1}\right\}\end{cases}
$$

$\left(c_{-m_{2}-1}^{(l)}=c_{m_{1}+1}^{(l)}=1\right)$ and the functions $F_{j}^{(l)}$ are defined by $(80)$.

Proof. By Theorem 6.4, we have limit in (65) along the whole sequence $\Lambda$. Reasoning as in the deduction of formulas (66) and (71), but now in connection with orthonormal polynomials (see Theorems 1 and 2 of [2]), it follows that

$$
\lim _{\mathbf{n} \in \Lambda} \frac{q_{\mathbf{n}^{l}, j}(z)}{q_{\mathbf{n}, j}(z)}= \begin{cases}\left(S_{j} \varphi_{j}\right)(z), & j \in\left\{-l_{2}, \ldots, l_{1}\right\}, \\ S_{j}(z), & j \in\left\{-m_{2}, \ldots,-l_{2}-1\right\} \cup\left\{l_{1}+1, \ldots, m_{1}\right\},\end{cases}
$$

uniformly on compact subsets of $\mathbb{C} \backslash \operatorname{supp}\left(\sigma_{j}\right)$, where $S_{j}$ is defined in (67). This formula, divided by (66) or (71) according to the value of $j$ gives

$$
\lim _{\mathbf{n} \in \Lambda} \frac{\kappa_{\mathbf{n}^{l}, j}}{\kappa_{\mathbf{n}, j}}=\sqrt{\omega_{j}}=\frac{c_{j}}{\sqrt{c_{j-1} c_{j+1}}},
$$

where $\omega_{j}$ is defined in (74), and the $c_{j}$ are the normalizing constants found in Lemma 6.3 solving the linear system of equations (76) which ensure that

$$
F_{j}^{(l)} \equiv c_{j} \widetilde{F}_{j}^{(l)}, \quad j=-m_{2}, \ldots, m_{1},
$$

with $F_{j}^{(l)}$ satisfying (64) and thus given by $(80)$. Since $\left(\widetilde{F}_{j}^{(l)}\right)^{\prime}(\infty)=1, j \in\left\{-l_{2}, \ldots, l_{1}\right\}$, and $\left(\widetilde{F}_{j}^{(l)}\right)(\infty)=1, j \in\left\{-m_{2}, \ldots,-l_{2}-1\right\} \cup\left\{l_{1}+1, \ldots, m_{1}\right\}$ formula (81) immediately follows with $\kappa_{j}^{(l)}$ as in $(84)$.

From the definition of $\kappa_{\mathbf{n}, j}$, we have that

$$
K_{\mathbf{n}, j}=\kappa_{\mathbf{n}, j} \cdots \kappa_{\mathbf{n}, m_{1}}
$$

Taking the ratio of these constants for the multi-indices $\mathbf{n}$ and $\mathbf{n}^{l}$ and using (81), we get (82). Formula (83) is an immediate consequence of (81) and (79).

Let $\operatorname{lcm}(a, b)$ denote the least common multiple of two integers $a$ and $b$, and define $m:=$ $\operatorname{lcm}\left(m_{1}+1, m_{2}+1\right), d_{1}:=m /\left(m_{1}+1\right), d_{2}:=m /\left(m_{2}+1\right)$. Within the class of pairs $l=\left(l_{1} ; l_{2}\right)$ with $0 \leq l_{1} \leq m_{1}, 0 \leq l_{2} \leq m_{2}$, we distinguish the subclass

$$
L:=\left\{\left(l_{1} ; l_{2}\right): l_{1} \equiv r \bmod \left(m_{1}+1\right), l_{2} \equiv r \bmod \left(m_{2}+1\right) \text { for some } 0 \leq r \leq m-1\right\}
$$

It is easy to check that for different $r, 0 \leq r \leq m-1$, the pairs $\left(l_{1}, l_{2}\right)$ in $L$ are different. Let $\mathbf{p}:=\left(\mathbf{p}_{1} ; \mathbf{p}_{2}\right)$, where $\mathbf{p}_{1}=\left(d_{1}, \ldots, d_{1}\right)$ and $\mathbf{p}_{2}=\left(d_{2}, \ldots, d_{2}\right)$ have $m_{1}+1$ and $m_{2}+1$ components, respectively. By $\mathbf{n}+\mathbf{p}$ we denote the multi-index $\left(\mathbf{n}_{1}+\mathbf{p}_{1} ; \mathbf{n}_{2}+\mathbf{p}_{2}\right)$. 
Corollary 6.6. Let $S^{1}=\mathcal{N}^{\prime}\left(\sigma_{0}^{1}, \ldots, \sigma_{m_{1}}^{1}\right), S^{2}=\mathcal{N}^{\prime}\left(\sigma_{0}^{2}, \ldots, \sigma_{m_{2}}^{2}\right)$ be given, and let $\Lambda \subset \mathbb{Z}_{+}^{m_{1}+1}(\bullet) \times$ $\mathbb{Z}_{+}^{m_{2}+1}(\bullet)$ be an infinite sequence of distinct multi-indices such that

$$
\sup _{\mathbf{n} \in \Lambda}\left(n_{1,0}-n_{1, m_{1}}\right)<\infty, \quad \sup _{\mathbf{n} \in \Lambda}\left(n_{2,0}-n_{1, m_{2}}\right)<\infty .
$$

Then, for each fixed $j \in\left\{-m_{2}, \ldots, m_{1}\right\}$, we have

$$
\begin{aligned}
& \lim _{\mathbf{n} \in \Lambda} \frac{Q_{\mathbf{n}+\mathbf{p}, j}(z)}{Q_{\mathbf{n}, j}(z)}=\prod_{l \in L} \widetilde{F}_{j}^{(l)}(z), \quad \mathcal{K} \subset \mathbb{C} \backslash \operatorname{supp}\left(\sigma_{j}\right), \\
& \lim _{\mathbf{n} \in \Lambda} \frac{\kappa_{\mathbf{n}+\mathbf{p}, j}}{\kappa_{\mathbf{n}, j}}=\prod_{l \in L} \kappa_{j}^{(l)}
\end{aligned}
$$

and

$$
\lim _{\mathbf{n} \in \Lambda} \frac{q_{\mathbf{n}+\mathbf{p}, j}(z)}{q_{\mathbf{n}, j}(z)}=\prod_{l \in L} \kappa_{j}^{(l)} \widetilde{F}_{j}^{(l)}(z), \quad \mathcal{K} \subset \mathbb{C} \backslash \operatorname{supp}\left(\sigma_{j}\right) .
$$

Proof. Given $\mathbf{n} \in \Lambda$ and $0 \leq r \leq m$, let $\mathbf{n}(r):=\mathbf{n}+\mathbf{q}(r)$ where $\mathbf{q}(r)=\left(\mathbf{q}_{1}(r) ; \mathbf{q}_{2}(r)\right)$ is the multi-index satisfying

$$
\mathbf{q}_{i}(r)=(\underbrace{k+1, \ldots, k+1}_{s}, k, \ldots, k), \quad r=k\left(m_{i}+1\right)+s, 0 \leq s \leq m_{i} .
$$

Hence, $\mathbf{n}(0)=\mathbf{n}, \mathbf{n}(m)=\mathbf{n}+\mathbf{p}$ and $\mathbf{n}(r) \in \mathbb{Z}_{+}^{m_{1}+1}(\bullet) \times \mathbb{Z}_{+}^{m_{2}+1}(\bullet)$ for every $r$.

We have

$$
\frac{Q_{\mathbf{n}+\mathbf{p}, j}(z)}{Q_{\mathbf{n}, j}(z)}=\prod_{r=0}^{m-1} \frac{Q_{\mathbf{n}(r+1), j}(z)}{Q_{\mathbf{n}(r), j}(z)} .
$$

In addition, by (79) we know that for each fixed $0 \leq r \leq m-1$,

$$
\lim _{\mathbf{n} \in \Lambda} \frac{Q_{\mathbf{n}(r+1), j}(z)}{Q_{\mathbf{n}(r), j}(z)}=\widetilde{F}_{j}^{(l)}(z), \quad z \in \mathcal{K} \subset \mathbb{C} \backslash \operatorname{supp}\left(\sigma_{j}\right),
$$

where $l=\left(l_{1} ; l_{2}\right)$ is precisely the multi-index satisfying $l_{1} \equiv r \bmod \left(m_{1}+1\right), l_{2} \equiv r \bmod \left(m_{2}+1\right)$. Therefore (85) follows. Relations (86) and (87) are proved analogously in view of (81) and (83).

Now, we need to introduce some notations. For $j \in\left\{-m_{2}, \ldots, m_{1}-1\right\}$, set

$$
\delta_{j}:=\left\{\begin{array}{ccccc}
1, & \text { if } & \Delta_{j} & \text { is to the left of } & \Delta_{j+1}, \\
-1, & \text { if } & \Delta_{j} & \text { is to the right of } & \Delta_{j+1} .
\end{array}\right.
$$

For multi-indices $l=\left(l_{1} ; l_{2}\right)$ such that $l_{1}+l_{2} \geq 2$ we define

$$
\Delta_{j, l}:=\left\{\begin{array}{ccc}
1, & \text { if } & j \geq l_{1}+2, \\
\delta_{j-1}, & \text { if } & j \in\left\{l_{1}, l_{1}+1\right\}, \\
-\delta_{j-1} \delta_{j}, & \text { if } & j \in\left\{-l_{2}+1, \ldots, l_{1}-1\right\}, \\
-\delta_{j}, & \text { if } & j \in\left\{-l_{2}-1,-l_{2}\right\}, \\
1, & \text { if } & j \leq-l_{2}-2 .
\end{array}\right.
$$


If $l_{1}+l_{2}=1$ then

and for $l_{1}=l_{2}=0$

$$
\Delta_{j, l}:=\left\{\begin{array}{ccc}
1, & \text { if } & j \geq l_{1}+2, \\
\delta_{j-1}, & \text { if } & j \in\left\{l_{1}, l_{1}+1\right\}, \\
-\delta_{j}, & \text { if } & j \in\left\{-l_{2}-1,-l_{2}\right\} \\
1, & \text { if } & j \leq-l_{2}-2,
\end{array}\right.
$$

$$
\Delta_{j,(0 ; 0)}:=\left\{\begin{array}{ccc}
1, & \text { if } & j \geq 2, \\
\delta_{0}, & \text { if } & j=1 \\
1, & \text { if } & j=0 \\
-\delta_{-1}, & \text { if } & j=-1, \\
1, & \text { if } & j \leq-2 .
\end{array}\right.
$$

Recall that $\varepsilon_{\mathbf{n}, j}$ denotes the sign of the varying measure

$$
d \rho_{\mathbf{n}, j}(x)=\frac{h_{\mathbf{n}, j}(x) d \sigma_{j}(x)}{Q_{\mathbf{n}, j-1}(x) Q_{\mathbf{n}, j+1}(x)} .
$$

Lemma 6.7. For any $\mathbf{n}, \mathbf{n}^{l} \in \mathbb{Z}_{+}^{m_{1}+1}(\bullet) \times \mathbb{Z}_{+}^{m_{2}+1}(\bullet)$ and $-m_{2} \leq j \leq m_{1}$

$$
\frac{\varepsilon_{\mathbf{n}^{l}, j}}{\varepsilon_{\mathbf{n}, j}}=\prod_{k=j}^{m_{1}} \Delta_{k, l}
$$

Proof. We will denote by $\operatorname{sign}(f, \Delta)$ the sign of a function $f$ on the interval $\Delta$. Thus

$$
\frac{\varepsilon_{\mathbf{n}^{l}, j}}{\varepsilon_{\mathbf{n}, j}}=\operatorname{sign}\left(\frac{\mathcal{H}_{\mathbf{n}^{l}, j} Q_{\mathbf{n}, j-1} Q_{\mathbf{n}, j+1}}{\mathcal{H}_{\mathbf{n}, j} Q_{\mathbf{n}^{l}, j-1} Q_{\mathbf{n}^{l}, j+1}}, \Delta_{j}\right) .
$$

If $-l_{2} \leq j-1 \leq l_{1}$, then $\operatorname{deg}\left(Q_{\mathbf{n}^{l}, j-1}\right)=1+\operatorname{deg}\left(Q_{\mathbf{n}, j-1}\right)$ and, therefore,

$$
\operatorname{sign}\left(\frac{Q_{\mathbf{n}, j-1}}{Q_{\mathbf{n}^{l}, j-1}}, \Delta_{j}\right)=\delta_{j-1} .
$$

If $j-1<-l_{2}$ or $j-1>l_{1}$, then $\operatorname{deg}\left(Q_{\mathbf{n}^{l}, j-1}\right)=\operatorname{deg}\left(Q_{\mathbf{n}, j-1}\right)$, implying that

$$
\operatorname{sign}\left(\frac{Q_{\mathbf{n}, j-1}}{Q_{\mathbf{n}^{l}, j-1}}, \Delta_{j}\right)=1 .
$$

Analogously, we have that for $-l_{2} \leq j+1 \leq l_{1}$

$$
\operatorname{sign}\left(\frac{Q_{\mathbf{n}, j+1}}{Q_{\mathbf{n}^{l}, j+1}}, \Delta_{j}\right)=-\delta_{j}
$$

and for $j+1<-l_{2}$ or $j+1>l_{1}$

$$
\operatorname{sign}\left(\frac{Q_{\mathbf{n}, j+1}}{Q_{\mathbf{n}^{l}, j+1}}, \Delta_{j}\right)=1 .
$$

From (90)-(92) it follows that

$$
\operatorname{sign}\left(\frac{Q_{\mathbf{n}, j-1} Q_{\mathbf{n}, j+1}}{Q_{\mathbf{n}^{l}, j-1} Q_{\mathbf{n}^{l}, j+1}}, \Delta_{j}\right)=\Delta_{j, l} .
$$

Now, by (53)

$$
\frac{\mathcal{H}_{\mathbf{n}^{l}, j}(x)}{\mathcal{H}_{\mathbf{n}, j}(x)}=\frac{\int \frac{Q_{\mathbf{n}^{l}, j+1}^{2}}{x-t}(t)}{\int \frac{\mathcal{H}_{\mathbf{n}^{l}, j+1}(t) d \sigma_{j+1}(t)}{Q_{\mathbf{n}^{l}, j}(t) Q_{\mathbf{n}^{l}, j+2}(t)}}
$$


Therefore,

$$
\operatorname{sign}\left(\mathcal{H}_{\mathbf{n}^{l}, j} / \mathcal{H}_{\mathbf{n}, j}, \Delta_{j}\right)=\frac{\varepsilon_{\mathbf{n}^{l}, j+1}}{\varepsilon_{\mathbf{n}, j+1}}
$$

Since $\mathcal{H}_{\mathbf{n}^{l}, m_{1}} \equiv \mathcal{H}_{\mathbf{n}, m_{1}} \equiv 1$, the right hand side of (95) equals 1 for $j=m_{1}$. Hence (88) follows from (89), (94) and (95).

This lemma shows that $\varepsilon_{\mathbf{n}^{l}, j} / \varepsilon_{\mathbf{n}, j}$ depends on $j, l$, and the relative positions of the intervals $\Delta_{j}$ but not on $\mathbf{n}$. Define the functions

$$
\mathcal{A}_{j}^{(l)}:=\widetilde{\psi}_{j}^{(l)} \prod_{k=j+1}^{m_{1}} \frac{\Delta_{k, l}}{\left(\kappa_{k}^{(l)}\right)^{2}}
$$

(the product should be understood to be equal to 1 when $j=m_{1}$ ).

Theorem 6.8. Let $S^{1}=\mathcal{N}^{\prime}\left(\sigma_{0}^{1}, \ldots, \sigma_{m_{1}}^{1}\right), S^{2}=\mathcal{N}^{\prime}\left(\sigma_{0}^{2}, \ldots, \sigma_{m_{2}}^{2}\right)$ be given, and let $\Lambda \subset \mathbb{Z}_{+}^{m_{1}+1}(\bullet) \times$ $\mathbb{Z}_{+}^{m_{2}+1}(\bullet)$ be an infinite sequence of distinct multi-indices such that

$$
\sup _{\mathbf{n} \in \Lambda}\left(n_{1,0}-n_{1, m_{1}}\right)<\infty, \quad \sup _{\mathbf{n} \in \Lambda}\left(n_{2,0}-n_{1, m_{2}}\right)<\infty .
$$

Let us assume that there exists $l=\left(l_{1} ; l_{2}\right), 0 \leq l_{1} \leq m_{1}, 0 \leq l_{2} \leq m_{2}$, such that for all $\mathbf{n} \in \Lambda$ we have that $\mathbf{n}^{l} \in \mathbb{Z}_{+}^{m_{1}+1}(\bullet) \times \mathbb{Z}_{+}^{m_{2}+1}(\bullet)$. Let $\left\{\mathcal{A}_{\mathbf{n}, j}\right\}_{j=-m_{2}-1}^{m_{1}}, \mathbf{n} \in \Lambda$, be the associated sequences of "monic" linear forms. Then, for each fixed $j=-m_{2}-1, \ldots, m_{1}$,

$$
\lim _{\mathbf{n} \in \Lambda} \frac{\mathcal{A}_{\mathbf{n}^{l}, j}(z)}{\mathcal{A}_{\mathbf{n}, j}(z)}=\mathcal{A}_{j}^{(l)}(z), \quad \mathcal{K} \subset \mathbb{C} \backslash\left(\operatorname{supp}\left(\sigma_{j}\right) \cup \operatorname{supp}\left(\sigma_{j+1}\right)\right)
$$

$\left(\operatorname{supp}\left(\sigma_{-m_{2}-1}\right)=\operatorname{supp}\left(\sigma_{m_{1}+1}\right)=\emptyset\right)$.

Proof. It follows from definition of $\mathcal{H}_{\mathbf{n}, j}$ and $\mathcal{H}_{\mathbf{n}^{l}, j}$ that

$$
\frac{\mathcal{A}_{\mathbf{n}^{l}, j}(z)}{\mathcal{A}_{\mathbf{n}, j}(z)}=\frac{\varepsilon_{\mathbf{n}^{l}, j+1} h_{\mathbf{n}^{l}, j}(z)}{\varepsilon_{\mathbf{n}, j+1} h_{\mathbf{n}, j}(z)} \frac{\varepsilon_{\mathbf{n}, j+1}}{\varepsilon_{\mathbf{n}^{l}, j+1}} \frac{K_{\mathbf{n}, j+1}^{2}}{K_{\mathbf{n}^{l}, j+1}^{2}} \frac{Q_{\mathbf{n}^{l}, j}(z)}{Q_{\mathbf{n}, j}(z)} \frac{Q_{\mathbf{n}, j+1}(z)}{Q_{\mathbf{n}^{l}, j+1}(z)} .
$$

By Lemma 6.1,

$$
\lim _{\mathbf{n} \in \Lambda} \frac{\varepsilon_{\mathbf{n}^{l}, j+1} h_{\mathbf{n}^{l}, j}(z)}{\varepsilon_{\mathbf{n}, j+1} h_{\mathbf{n}, j}(z)}=1, \quad \mathcal{K} \subset \mathbb{C} \backslash \operatorname{supp}\left(\sigma_{j+1}\right) .
$$

Using Lemma 6.7 and Corollary 6.5, we have

$$
\lim _{\mathbf{n} \in \Lambda} \frac{\varepsilon_{\mathbf{n}, j+1}}{\varepsilon_{\mathbf{n}^{l}, j+1}} \frac{K_{\mathbf{n}, j+1}^{2}}{K_{\mathbf{n}^{l}, j+1}^{2}}=\prod_{k=j+1}^{m_{1}} \frac{\Delta_{k, l}}{\left(\kappa_{k}^{(l)}\right)^{2}}
$$

Finally, applying (79) and (80) one obtains

$$
\lim _{\mathbf{n} \in \Lambda} \frac{Q_{\mathbf{n}^{l}, j}(z)}{Q_{\mathbf{n}, j}(z)} \frac{Q_{\mathbf{n}, j+1}(z)}{Q_{\mathbf{n}^{l}, j+1}(z)}=\widetilde{\psi}_{j}^{(l)}(z), \quad \mathcal{K} \subset \mathbb{C} \backslash\left(\operatorname{supp}\left(\sigma_{j}\right) \cup \operatorname{supp}\left(\sigma_{j+1}\right)\right) .
$$

Putting these relations together we get (97). 
Corollary 6.9. Let $S^{1}=\mathcal{N}^{\prime}\left(\sigma_{0}^{1}, \ldots, \sigma_{m_{1}}^{1}\right), S^{2}=\mathcal{N}^{\prime}\left(\sigma_{0}^{2}, \ldots, \sigma_{m_{2}}^{2}\right)$ be given, and let $\Lambda \subset \mathbb{Z}_{+}^{m_{1}+1}(\bullet) \times$ $\mathbb{Z}_{+}^{m_{2}+1}(\bullet)$ be an infinite sequence of distinct multi-indices such that

$$
\sup _{\mathbf{n} \in \Lambda}\left(n_{1,0}-n_{1, m_{1}}\right)<\infty, \quad \sup _{\mathbf{n} \in \Lambda}\left(n_{2,0}-n_{1, m_{2}}\right)<\infty .
$$

Then, for each fixed $j \in\left\{-m_{2}, \ldots, m_{1}\right\}$, we have

$$
\lim _{\mathbf{n} \in \Lambda} \frac{\mathcal{A}_{\mathbf{n}+\mathbf{p}, j}(z)}{\mathcal{A}_{\mathbf{n}, j}(z)}=\prod_{l \in L} \mathcal{A}_{j}^{(l)}(z), \quad \mathcal{K} \subset \mathbb{C} \backslash\left(\operatorname{supp}\left(\sigma_{j}\right) \cup \operatorname{supp}\left(\sigma_{j+1}\right)\right)
$$

$\left(\operatorname{supp}\left(\sigma_{-m_{2}-1}\right)=\operatorname{supp}\left(\sigma_{m_{1}+1}\right)=\emptyset\right)$. Consequently

$$
\lim _{\mathbf{n} \in \Lambda}\left|\mathcal{A}_{\mathbf{n}, j}(z)\right|^{1 /\left|\mathbf{n}_{1}\right|}=\prod_{l \in L}\left|\mathcal{A}_{j}^{(l)}(z)\right|^{1 / m}, \quad \mathcal{K} \subset \mathbb{C} \backslash\left(\operatorname{supp}\left(\sigma_{j}\right) \cup \operatorname{supp}\left(\sigma_{j+1}\right)\right),
$$

where $m=\operatorname{lcm}\left(m_{1}+1, m_{2}+1\right)$.

Proof. Using the same arguments employed to prove Corollary 6.6, we obtain (99). From (99) it is easy to deduce the $\left|\mathbf{n}_{1}\right|$-th root asymptotics of the linear forms.

In fact, it is easy to see that for each $\mathbf{n} \in \Lambda$ there exists $\mathbf{n}_{0} \in \mathbb{Z}_{+}^{m_{1}+1}(\bullet) \times \mathbb{Z}_{+}^{m_{2}+1}(\bullet)$ (which may depend on $\mathbf{n}$ ), whose entries are uniformly bounded by a constant $C$ independent of $\mathbf{n}$ (condition (98) is used), such that $\mathbf{n}=r \mathbf{p}+\mathbf{n}_{0}$ for some $r \in \mathbb{Z}_{+}$. Write

$$
\mathcal{A}_{\mathbf{n}, j}(z)=\frac{\mathcal{A}_{\mathbf{n}, j}(z)}{\mathcal{A}_{\mathbf{n}-\mathbf{p}, j}(z)} \frac{\mathcal{A}_{\mathbf{n}-\mathbf{p}, j}(z)}{\mathcal{A}_{\mathbf{n}-2 \mathbf{p}, j}(z)} \cdots \frac{\mathcal{A}_{\mathbf{n}_{0}+\mathbf{p}, j}(z)}{\mathcal{A}_{\mathbf{n}_{0}, j}(z)} \mathcal{A}_{\mathbf{n}_{0}, j}(z) .
$$

Then

$$
\frac{1}{\left|\mathbf{n}_{1}\right|} \log \left|\mathcal{A}_{\mathbf{n}, j}(z)\right|=\frac{1}{\left|\mathbf{n}_{1}\right|} \log \left|\mathcal{A}_{\mathbf{n}_{0}, j}(z)\right|+\frac{1}{\left|\mathbf{n}_{1}\right|} \sum_{k=0}^{r-1} \log \left|\frac{\mathcal{A}_{\mathbf{n}_{0}+(k+1) \mathbf{p}, j}(z)}{\mathcal{A}_{\mathbf{n}_{0}+k \mathbf{p}, j}(z)}\right| .
$$

Obviously,

$$
\lim _{\mathbf{n} \in \Lambda} \frac{1}{\left|\mathbf{n}_{1}\right|} \log \left|\mathcal{A}_{\mathbf{n}_{0}, j}(z)\right|=0, \quad \mathcal{K} \subset \mathbb{C} \backslash\left(\operatorname{supp}\left(\sigma_{j}\right) \cup \operatorname{supp}\left(\sigma_{j+1}\right)\right),
$$

and because of $(99)$

$$
\lim _{\mathbf{n} \in \Lambda} \frac{1}{\left|\mathbf{n}_{1}\right|} \sum_{k=0}^{r-1} \log \left|\frac{\mathcal{A}_{\mathbf{n}_{0}+(k+1) \mathbf{p}, j}(z)}{\mathcal{A}_{\mathbf{n}_{0}+k \mathbf{p}, j}(z)}\right|=\frac{1}{m} \log \left|\prod_{l \in L} \mathcal{A}_{j}^{(l)}(z)\right|, \quad \mathcal{K} \subset \mathbb{C} \backslash\left(\operatorname{supp}\left(\sigma_{j}\right) \cup \operatorname{supp}\left(\sigma_{j+1}\right)\right),
$$

since $\left|\mathbf{n}_{1}\right|=r\left|\mathbf{p}_{1}\right|+\mathcal{O}(1)=r m+\mathcal{O}(1),\left|\mathbf{n}_{1}\right| \rightarrow \infty$.

The function appearing on the right hand side of (100) corresponds with the one on the right hand side of (40) associated to the vector equilibrium problem with interaction matrix $\mathcal{C}$ constructed taking $p_{1, k}=1 /\left(m_{1}+1\right), 0 \leq k \leq m_{1}$, and $p_{2, k}=1 /\left(m_{2}+1\right), 0 \leq k \leq m_{2}$. In that case, for each $j=-m_{2}-1, \ldots, m_{1}$, we have

$$
G_{j}(z)=\prod_{l \in L}\left|\mathcal{A}_{j}^{(l)}(z)\right|^{1 / m}, \quad z \in \mathbb{C} \backslash\left(\widetilde{\Delta}_{j} \cup \widetilde{\Delta}_{j+1}\right)
$$

$\left(\Delta_{-m_{2}-1}=\Delta_{m_{1}+1}=\emptyset\right)$, where $m=\operatorname{lcm}\left(m_{1}+1, m_{2}+1\right)$. 


\section{Application to mixed type Hermite Padé approximation}

Let $S^{1}=\mathcal{N}\left(\sigma_{0}^{1}, \ldots, \sigma_{m_{1}}^{1}\right), S^{2}=\mathcal{N}\left(\sigma_{0}^{2}, \ldots, \sigma_{m_{2}}^{2}\right), \sigma_{0}^{1}=\sigma_{0}^{2}$ be given. Let us introduce the row vectors

$$
\mathbb{U}=\left(1, \widehat{s}_{1,1}^{2}, \ldots, \widehat{s}_{1, m_{2}}^{2}\right), \quad \mathbb{V}=\left(1, \widehat{s}_{1,1}^{1}, \ldots, \widehat{s}_{1, m_{1}}^{1}\right)
$$

and the $\left(m_{2}+1\right) \times\left(m_{1}+1\right)$ dimensional matrix

$$
\mathbb{W}=\mathbb{U}^{t} \mathbb{V}
$$

where the super-index $t$ means taking transpose. Define the matrix Markov type function

$$
\widehat{\mathbb{S}}(z)=\int \frac{\mathbb{W}(x) d \sigma_{0}^{2}(x)}{z-x}
$$

understanding that integration is carried out entry by entry on the matrix $\mathbb{W}$.

Fix $\mathbf{n}_{1}=\left(n_{1,0}, n_{1,1}, \ldots, n_{1, m_{1}}\right) \in \mathbb{Z}_{+}^{m_{1}+1}$ and $\mathbf{n}_{2}=\left(n_{2,0}, n_{2,1}, \ldots, n_{2, m_{2}}\right) \in \mathbb{Z}_{+}^{m_{2}+1},\left|\mathbf{n}_{2}\right|=$ $\left|\mathbf{n}_{1}\right|-1$. It is easy to see that there exists a non zero vector polynomial

$$
\mathbb{A}_{\mathbf{n}}=\left(a_{\mathbf{n}, 0}, \ldots, a_{\mathbf{n}, m_{1}}\right), \quad \operatorname{deg}\left(a_{\mathbf{n}, k}\right) \leq n_{1, k}-1, \quad k=0, \ldots, m_{1},
$$

such that

$$
\widehat{\mathbb{S}}(z) \mathbb{A}_{\mathbf{n}}^{t}(z)-\mathbb{D}_{\mathbf{n}}^{t}(z)=\left(\mathcal{O}\left(1 / z^{n_{2,0}+1}\right), \ldots, \mathcal{O}\left(1 / z^{n_{2, m_{2}}+1}\right)\right)^{t}=: \mathcal{O}\left(1 / z^{\mathbf{n}_{2}+1}\right), \quad z \rightarrow \infty,
$$

where $\mathbb{D}_{\mathbf{n}}=\left(d_{\mathbf{n}, 0}, \ldots, d_{\mathbf{n}, m_{2}}\right)$ is some vector polynomial. When $m_{2}=0$, this construction is called type I Hermite-Padé approximation. If $m_{1}=0$ it is called of type II. When $m_{1}=m_{2}=0$ it reduces to diagonal Padé approximation. This definition is of mixed type.

Lemma 7.1. For $j=0, \ldots, m_{2},\left(\widehat{s}_{1,0}^{1} \equiv 1\right)$

$$
\int x^{\nu} \sum_{k=0}^{m_{1}} a_{\mathbf{n}, k}(x) \widehat{s}_{1, k}^{1}(x) d s_{j}^{2}(x)=0, \quad \nu=0, \ldots, n_{2, j}-1 .
$$

Proof. In fact, notice that according to (101), for each $\nu, 0 \leq \nu \leq n_{2, j}-1, j=0, \ldots, m_{2}$,

$$
z^{\nu}\left(\sum_{k=0}^{m_{1}} a_{\mathbf{n}, k}(z) \int \frac{\widehat{s}_{1, j}^{2}(x) \widehat{s}_{1, k}^{1}(x) d \sigma_{0}^{2}(x)}{z-x}-d_{\mathbf{n}, j}(z)\right)=\mathcal{O}\left(1 / z^{2}\right), \quad z \rightarrow \infty,
$$

$\left(\widehat{s}_{1,0}^{2} \equiv 1\right)$ and the function on the left hand side is holomorphic in $\overline{\mathbb{C}} \backslash \operatorname{Co}\left(\operatorname{supp}\left(\sigma_{0}^{2}\right)\right)$. Using Lemma 2.4 , we obtain (102).

Because of this Lemma, we see that $\mathbb{A}_{\mathbf{n}}$ is an $\mathbf{n}$-th mixed type multiple orthogonal polynomial with respect to the pair $\left(S^{1}, S^{2}\right)$ and in the sequel we assume that it is "monic". If

$$
\mathbb{B}_{\mathbf{n}}=\left(b_{\mathbf{n}, 0}, \ldots, b_{\mathbf{n}, m_{2}}\right), \quad \operatorname{deg}\left(b_{\mathbf{n}, j}\right) \leq n_{2, j}-1, \quad j=0, \ldots, m_{2},
$$

denotes a generic vector polynomial with the indicated degrees, (102) may be rewritten in matrix form as

$$
\int \mathbb{B}_{\mathbf{n}}(x) \mathbb{W}(x) \mathbb{A}_{\mathbf{n}}^{t}(x) d \sigma_{0}^{2}(x)=0, \quad \text { for all } \mathbb{B}_{\mathbf{n}}
$$


Fix $j \in\left\{0, \ldots, m_{2}\right\}$. For each $k \in\{-1, \ldots,-j-1\}$ define

$$
\Omega_{k}^{j}=\left\{z \in \mathbb{C} \backslash \cup_{i=0}^{-j-1} \Delta_{i}: U_{k}^{\bar{\mu}}(z)<U_{i}^{\bar{\mu}}(z), i=-1, \ldots,-j-1, i \neq k\right\}, \quad \Omega_{-1}^{0}=\mathbb{C} \backslash\left(\Delta_{0} \cup \Delta_{-1}\right) .
$$

Set

$$
\chi_{j}(z):=\min \left\{U_{k}^{\bar{\mu}}(z): k=-1, \ldots,-j-1\right\}
$$

and

$$
\left(\mathcal{R}_{\mathbf{n}, 0}, \ldots, \mathcal{R}_{\mathbf{n}, m_{2}}\right)^{t}:=\widehat{\mathbb{S}}(z) \mathbb{A}_{\mathbf{n}}^{t}(z)-\mathbb{D}_{\mathbf{n}}^{t}(z)
$$

Theorem 7.2. Let $\Lambda=\Lambda\left(p_{1,0}, \ldots, p_{1, m_{1}} ; p_{2,0}, \ldots, p_{2, m_{2}}\right) \subset \mathbb{Z}_{+}^{m_{1}+1}(\bullet) \times \mathbb{Z}_{+}^{m_{2}+1}(\bullet),\left(S^{1}, S^{2}\right) \in \mathbf{R e g}$, $S^{1}=\mathcal{N}\left(\sigma_{0}^{1}, \ldots, \sigma_{m_{1}}^{1}\right)$, and $S^{2}=\mathcal{N}\left(\sigma_{0}^{2}, \ldots, \sigma_{m_{2}}^{2}\right)$ be given. Then for each $j \in\left\{0, \ldots, m_{2}\right\}$

$$
\lim _{\mathbf{n} \in \Lambda}\left|\mathcal{R}_{\mathbf{n}, j}(z)\right|^{1 /\left|\mathbf{n}_{1}\right|}=\exp \left(-\chi_{j}(z)\right), \quad \mathcal{K} \subset \cup_{k=-1}^{-j-1} \Omega_{k}^{j}
$$

and

$$
\lim _{\mathbf{n} \in \Lambda}\left|\mathcal{R}_{\mathbf{n}, j}(z)\right|^{1 /\left|\mathbf{n}_{1}\right|} \leq \exp \left(-\chi_{j}(z)\right), \quad \mathcal{K} \subset \mathbb{C} \backslash\left(\cup_{k=0}^{-j-1} \Delta_{k}\right) .
$$

In particular, if $p_{2,0}=\cdots=p_{2, m_{2}}=1 /\left(m_{2}+1\right)$, then

$$
\lim _{\mathbf{n} \in \Lambda}\left|\mathcal{R}_{\mathbf{n}, j}(z)\right|^{1 /\left|\mathbf{n}_{1}\right|}=\exp \left(-U_{-1}^{\bar{\mu}}(z)\right), \quad \mathcal{K} \subset \mathbb{C} \backslash\left(\cup_{k=0}^{-j-1} \Delta_{k}\right) .
$$

$\bar{\mu}=\bar{\mu}(\mathcal{C})=\left(\bar{\mu}_{-m_{2}}, \ldots, \bar{\mu}_{m_{1}}\right)$ is the equilibrium vector measure and $\left(\omega_{-m_{2}}^{\bar{\mu}}, \ldots, \omega_{m_{1}}^{\bar{\mu}}\right)$ is the system of equilibrium constants for the vector potential problem determined by the interaction matrix $\mathcal{C}$ defined in (4) on the system of compact sets $E_{k}=\operatorname{supp}\left(\sigma_{k}^{1}\right), k=0, \ldots, m_{1}, E_{k}=\operatorname{supp}\left(\sigma_{-k}^{2}\right), k=$ $-m_{2}, \ldots, 0$.

Proof. Notice that (103) implies that

$$
\widehat{\mathbb{S}}(z) \mathbb{A}_{\mathbf{n}}^{t}(z)-\int \frac{\mathbb{W}(x)\left(\mathbb{A}_{\mathbf{n}}^{t}(z)-\mathbb{A}_{\mathbf{n}}^{t}(x)\right) d \sigma_{0}^{2}(x)}{z-x}=\int \frac{\mathbb{W}(x) \mathbb{A}_{\mathbf{n}}^{t}(x) d \sigma_{0}^{2}(x)}{z-x}=\mathcal{O}\left(1 / z^{\mathbf{n}_{2}+1}\right), \quad z \rightarrow \infty,
$$

and taking

$$
\mathbb{D}_{\mathbf{n}}^{t}(z)=\int \frac{\mathbb{W}(x)\left(\mathbb{A}_{\mathbf{n}}^{t}(z)-\mathbb{A}_{\mathbf{n}}^{t}(x)\right) d \sigma_{0}^{2}(x)}{z-x}
$$

we obtain an integral expression for the remainder in (101).

Then

$$
\left(\mathcal{R}_{\mathbf{n}, 0}(z), \ldots, \mathcal{R}_{\mathbf{n}, m_{2}}(z)\right)^{t}=\int \frac{\mathbb{W}(x) \mathbb{A}_{\mathbf{n}}^{t}(x) d \sigma_{0}^{2}(x)}{z-x} .
$$

In scalar form this says that

$$
\mathcal{R}_{\mathbf{n}, j}(z)=\int \frac{\mathcal{A}_{\mathbf{n}, 0}(x)}{z-x} d s_{j}^{2}(x), \quad j=0, \ldots, m_{2} .
$$

Notice that (see (13))

$$
\mathcal{R}_{\mathbf{n}, 0}(z)=\mathcal{A}_{\mathbf{n},-1}(z) .
$$

Let us establish a connection between the remainders $\mathcal{R}_{\mathbf{n}, j}(z)$ and the forms $\mathcal{A}_{\mathbf{n}, k}(z)$ with negative indices $k \in\{-1, \ldots,-j-1\}$. 
Fix $j \in\left\{1, \ldots, m_{2}\right\}$. We have

$$
(-1)^{j} \mathcal{R}_{\mathbf{n}, j}(z)=\int \cdots \int \frac{\mathcal{A}_{\mathbf{n}, 0}\left(x_{0}\right) d \sigma_{0}^{2}\left(x_{0}\right) \cdots d \sigma_{j}^{2}\left(x_{j}\right)}{\left(z-x_{0}\right)\left(x_{1}-x_{0}\right) \cdots\left(x_{j}-x_{j-1}\right)},
$$

and

Consequently,

$$
\mathcal{A}_{\mathbf{n},-j-1}(z)=\int \cdots \int \frac{\mathcal{A}_{\mathbf{n}, 0}\left(x_{0}\right) d \sigma_{0}^{2}\left(x_{0}\right) \cdots d \sigma_{j}^{2}\left(x_{j}\right)}{\left(x_{1}-x_{0}\right) \cdots\left(x_{j}-x_{j-1}\right)\left(z-x_{j}\right)}
$$

$$
(-1)^{j} \mathcal{R}_{\mathbf{n}, j}(z)-\mathcal{A}_{\mathbf{n},-j-1}(z)=\int \cdots \int \frac{-\left(x_{j}-x_{0}\right) \mathcal{A}_{\mathbf{n}, 0}\left(x_{0}\right) d \sigma_{0}^{2}\left(x_{0}\right) \cdots d \sigma_{j}^{2}\left(x_{j}\right)}{\left(z-x_{0}\right)\left(x_{1}-x_{0}\right) \cdots\left(x_{j}-x_{j-1}\right)\left(z-x_{j}\right)} .
$$

Since $x_{j}-x_{0}=x_{j}-x_{j-1}+x_{j-1}-\cdots-x_{1}+x_{1}-x_{0}$, substituting this in the previous formula, we obtain

$$
\mathcal{A}_{\mathbf{n},-j-1}(z)=\sum_{k=0}^{j-1}(-1)^{k}\left\langle\sigma_{j}^{2}, \ldots, \sigma_{k+1}^{2} \hat{\gamma}(z) \mathcal{R}_{\mathbf{n}, k}(z)+(-1)^{j} \mathcal{R}_{\mathbf{n}, j}(z) .\right.
$$

We have a triangular scheme of linear equations whose coefficients do not depend on $\mathbf{n}$. We can solve for $\mathcal{R}_{\mathbf{n}, j}$ in terms of $\mathcal{A}_{\mathbf{n},-1}, \ldots, \mathcal{A}_{\mathbf{n},-j-1}$. Using (50) one obtains that for each $j \in\left\{0, \ldots, m_{2}\right\}$ (when $j=0$ the sum below is empty)

$$
\mathcal{R}_{\mathbf{n}, j}(z)=\sum_{k=1}^{j}(-1)^{k-1}\left\langle\sigma_{k}^{2}, \ldots, \sigma_{j}^{2} \widehat{\gamma}(z) \mathcal{A}_{\mathbf{n},-k}(z)+(-1)^{j} \mathcal{A}_{\mathbf{n},-j-1}(z) .\right.
$$

Taking (40) into consideration, on $\Omega_{-k}^{j}$ the term containing $\mathcal{A}_{\mathbf{n},-k}$ dominates the sum (notice that $\left.\left\langle\sigma_{k}^{2}, \ldots, \sigma_{j}^{2}\right\rangle(z) \neq 0, z \in \mathbb{C} \backslash \Delta_{-k}\right)$ and (104) immediately follows. On the complement of $\cup_{k=-1}^{-j-1} \Omega_{k}^{j}$ there is no dominating term and all we can conclude from the previous equality is (105).

Let $p_{2,0}=\cdots=p_{2, m_{2}}=1 /\left(m_{2}+1\right)$. In this case, on $\mathbb{C} \backslash \cup_{k=0}^{-j-1} \Delta_{k}$ we have that $U_{-1}^{\bar{\mu}}(z)<$ $U_{-2}^{\bar{\mu}}(z)<\cdots<U_{-j-1}^{\bar{\mu}}(z)$ (see third sentence before Corollary 5.3) and (106) follows from (104).

Remark 7.3. Fix $j \in\left\{0, \ldots, m_{2}\right\}$. For each $k \in\{-1, \ldots,-j-1\}$ we could have defined

$$
\Omega_{k}^{j}=\left\{z \in \mathbb{C} \backslash \cup_{i=0}^{-j-1} E_{i}: U_{k}^{\bar{\mu}}(z)<U_{i}^{\bar{\mu}}(z), i=-1, \ldots,-j-1, i \neq k\right\}, \quad \Omega_{-1}^{0}=\mathbb{C} \backslash\left(E_{0} \cup E_{-1}\right) .
$$

Taking into account that the polynomials $Q_{\mathbf{n}, i}$ and the forms $\mathcal{A}_{\mathbf{n}, i}$ may have at most one zero in each of the connected components of $\Delta_{i} \backslash E_{i}$, one can prove in place of (104)-(106) convergence in capacity on each compact subset of the corresponding regions.

We say that $\mathcal{I}_{1} \subset \mathbb{Z}_{+}^{m_{1}+1}(\bullet)$ is a complete, ordered, sequence of multi-indices if:

a) For each $n \in \mathbb{Z}_{+}$, there exists a unique $\mathbf{n}_{1} \in \mathcal{I}_{1}$ such that $\left|\mathbf{n}_{1}\right|=n$.

b) Any two multi-indices in $\mathcal{I}_{1}$ are ordered in the sense that all components of one of them are less than or equal to the corresponding components of the other one, or they are identical.

Fix $\mathcal{I}_{1} \subset \mathbb{Z}_{+}^{m_{1}+1}(\bullet), \mathcal{I}_{2} \subset \mathbb{Z}_{+}^{m_{2}+1}(\bullet)$, two complete, ordered sequences of multi-indices. Each $n \in \mathbb{Z}_{+}$determines a unique $\mathbf{n}_{1} \in \mathcal{I}_{1}$ and $\mathbf{n}_{2} \in \mathcal{I}_{2}$ such that $n=\left|\mathbf{n}_{1}\right|=\left|\mathbf{n}_{2}\right|+1$. The corresponding "monic" mixed type multiple orthogonal polynomials we denote by $\mathbb{A}_{n}$. We can interchange the roles of the Nikishin systems $S^{1}, S^{2}$, and determine a sequence of "monic" mixed type multiple 
orthogonal polynomials which we denote $\mathbb{B}_{n}$. It is easy to verify that the sequences $\left\{\mathbb{A}_{n}\right\},\left\{\mathbb{B}_{n}\right\}, n \in$ $\mathbb{Z}_{+}$are bi-orthogonal. That is,

$$
\int \mathbb{B}_{n^{\prime}}(x) \mathbb{W}(x) \mathbb{A}_{n}^{t}(x) d \sigma_{0}^{2}(x) \begin{cases}=0, & n \neq n^{\prime} \\ \neq 0, & n=n^{\prime}\end{cases}
$$

The inequality in (107) is a consequence of Lemma 2.2. With the same hypothesis, all the results of this paper hold true for the sequence $\left\{\mathbb{B}_{n}\right\}, n \in \mathbb{Z}_{+}$.

\section{REFERENCES}

[1] A.I. Aptekarev, G. López Lagomasino and I.A. Rocha, Ratio asymptotic of Hermite-Padé orthogonal polynomials for Nikishin systems, Sbornik: Mathematics 196 (2005), 1089-1107.

[2] D. Barrios, B. de la Calle and G. López Lagomasino, Ratio and relative asymptotics of polynomials orthogonal with respect to varying Denisov type measures, J. of Approx. Theory 139 (2006), 223-256.

[3] M. Bello Hernández, G. López Lagomasino and J. Mínguez Ceniceros, Fourier-Padé approximants for Angelesco systems, Constr. Approx. 26 (2007), 339-359.

[4] B. de la Calle Ysern and G. López Lagomasino, Weak convergence of varying measures and Hermite-Padé orthogonal polynomials, Constr. Approx. 15 (1999), 553-575.

[5] F. Cala Rodríguez and G. López Lagomasino, Multipoint rational approximation with preassigned poles, J. of Math. Anal. and Appl. 256 (2001), 142-161.

[6] E. Daems and A.B.J. Kuijlaars, Multiple orthogonal polynomials of mixed type and non-intersecting brownian motions, J. of Approx. Theory. 146 (2007), 91-114.

[7] S. A. Denisov, On Rakhmanov's theorem for Jacobi matrices, Proc. Amer. Math. Soc. 132 (2004), 847-852.

[8] U. Fidalgo Prieto and G. López Lagomasino, Rate of convergence of generalized Hermite-Padé approximants of Nikishin systems, Constr. Approx. 23 (2006), 165-196.

[9] U. Fidalgo Prieto and G. López Lagomasino, On perfect Nikishin systems, Comput. Methods and Function Theory 2 (2002), 415-426.

[10] A. A. Gonchar and E. A. Rakhmanov, The equilibrium measure and distribution of zeros of extremal polynomials, Math. USSR Sb. 53 (1986), 119-130.

[11] A.A. Gonchar, E.A. Rakhmanov, and V.N. Sorokin, Hermite-Padé approximants for systems of Markov-type functions, Sbornik: Mathematics 188 (1997), 33-58.

[12] G. Lopes [G. López Lagomasino], On the asymptotic of the ratio of orthogonal polynomials and convergence of multipoint Padé approximants, Math. USSR Sb. 56 (1987), 207-220.

[13] G. Lopes [G. López Lagomasino], Convergence of Padé approximants of Stieltjes type meromorphic functions and comparative asymptotic of orthogonal polynomials, Math. USSR Sb. 64 (1989), 207-227.

[14] A. López García and G. López Lagomasino, Ratio asymptotic of Hermite-Padé orthogonal polynomials for Nikishin systems. II, Adv. in Math. 218 (2008), 1081-1106.

[15] G. López Lagomasino and J. Mínguez Ceniceros, Fourier-Padé approximants for Nikishin systems, Constr. Approx. 30 (2009), 53-69.

[16] P. Nevai, Weakly convergent sequences of functions and orthogonal polynomials, J. of Approx. Theory 65 (1991), 322-340.

[17] P. Nevai and V. Totik, Denisov's theorem on recurrence coefficients, J. of Approx. Theory 127 (2004), 240-245.

[18] E.M. Nikishin, On simultaneous Padé approximants, Math. USSR Sb. 41 (1982), 409-425. 
[19] E.M. Nikishin, Asymptotics of linear forms for simultaneous Padé approximants, Soviet Math. (Iz. VUZ) 30, (1986).

[20] E.M. Nikishin and V.N. Sorokin, Rational Approximations and Orthogonality, Transl. of Math. Monographs Vol. 92, Amer. Math. Soc., Providence, Rhode Island, 1991.

[21] E.A. Rakhmanov, On the asymptotic of the ratio of orthogonal polynomials, Math. USSR Sb. 32 (1977), 199-213.

[22] E.A. Rakhmanov, On the asymptotic of the ratio of orthogonal polynomials II, Math. USSR Sb. 46 (1983), 105-117.

[23] E.A. Rakhmanov, On asymptotic properties of orthogonal polynomials on the unit circle with weights not satisfying Szegő's condition, Math. USSR Sb. 58 (1987), 149-167.

[24] V.N. Sorokin, On simultaneous approximation of several linear forms, Vestnik Mosk. Univ., Ser. Matem., Mekh., (1983), No. 1, 44-47.

[25] V.N. Sorokin, Hermite-Padé approximants for polylogarithms, Russian Mathematics (Iz. VUZ) 38 (1994), $47-57$.

[26] V.N. Sorokin and J. Van Iseghem, Algebraic aspects of matrix orthogonality for vector polynomials, J. of Approx. Theory 90 (1997), 97-116.

[27] E.B. Saff and V. Totik, Logarithmic Potentials with External Fields, Series of Comprehensive Studies in Mathematics, Vol. 316, Springer, New York, 1997.

[28] H. Stahl and V. Totik, General Orthogonal Polynomials, Enc. Math. Vol. 43, Cambridge University Press, Cambridge, 1992.

U. Fidalgo Prieto, Dpto. de Matemáticas, Universidad Carlos iil de Madrid, C/ Universidad 30, 28911 Leganés, SpAin.

E-mail address: ufidalgo@math.uc3m.es

A. López García, Department of Mathematics, Vanderbilt University, Nashville, tN 37240, USA.

E-mail address: abey.lopez@Vanderbilt.edu

G. López Lagomasino, Dpto. de Matemáticas, Universidad Carlos iII de Madrid, C/ Universidad 30, 28911, Leganés, Spain.

E-mail address: lago@math.uc3m.es

V. N. Sorokin, Dept. of Function Theory and Functional Analysis, Moscow State University, 119992 Leninskye Gory, Moscow, Russia.

E-mail address: vnsormm@mech.math.msu.su 